\title{
A Synthetic Study towards Saccharomicin Based upon Asymmetric Metal Catalysis
}

\author{
Supporting Information \\ Bhawna Barpuzary, Mijin Kim and Young Ho Rhee* \\ Department of Chemistry, Pohang University of Science and Technology (POSTECH), Hyoja-dong San 31, Pohang 790-784, \\ Republic of Korea
}

\section{Table of Contents}

1. General Information

2. Synthesis of substrates $\mathbf{1}, \mathbf{5}$ and $\mathbf{1 1}$

3. Synthesis of cyclic 0,0 -acetal $7 \mathbf{a}$ and $7 \mathbf{b}$

4. Synthesis of C3-methyl amino sugar - D-saccharosamine cyclohexyl glycoside $\mathbf{1 0}$

5. Synthesis of $\mathbf{1 3 a}$ and $\mathbf{1 3 b}$ via Pd-catalyzed hydroalkoxylation followed by Ring-Closing Metathesis

6. Synthesis of 3-amino-3-methyl disaccharide unit $\mathbf{1 7}$ of Saccharomicin

7. Synthesis of Fucose-Rhamnose disaccharide $\mathbf{2 3}$ by protective group-less coupling

8. Structure determination of compound $7 \mathbf{b}$ by $1 H$ NMR and $2 D N M R$ of dihydroxylated product

9. Structure determination of compound $\mathbf{S} 8$ and 22 by $2 \mathrm{D}$ NMR

10. References

11. Ratios of acyclic and cyclic $O, O$-acetals; determined by crude ${ }^{1} \mathrm{H} N M R$

12. ${ }^{1} \mathrm{H}$ and ${ }^{13} \mathrm{C}$ NMR of new compounds 


\section{General Information}

Air and moisture sensitive reactions were carried out in oven-dried glassware sealed with rubber septa under a positive pressure of nitrogen. Similarly, all solvents were dried and distilled according to the standard methods before use, then were transferred via syringe. Reactions were stirred using Teflon-coated magnetic stir bars. $\mathrm{Pd}_{2}(\mathrm{dba})_{3}$, the Grubbs' catalysts were purchased form a Aldrich Chemical, Strem Chemical Inc. Chiral Trost ligands were purchased from Strem Chemical Inc. and stored in glove box. Reactions were monitored by thin-layer chromatography on silicagel carried out on $0.25 \mathrm{~mm}$ E. Merck silica gel plates (60F- 254) using UV light as a visualizing agent and acidic $p$-anisaldehyde, and heat as developing agent. Flash chromatography on silicagel was carried out on Merck 60 silica gel (230-400 mesh). ${ }^{1} \mathrm{H}$ and ${ }^{13} \mathrm{C}$ NMR spectra were recorded on Bruker $(300 \mathrm{MHz}, 500 \mathrm{MHz})$ spectrometer. ${ }^{1} \mathrm{H}$ NMR spectra were referenced to $\mathrm{CDCl}_{3}(7.26 \mathrm{ppm})$, and reported as follows; chemical shift, multiplicity $(s=$ singlet, $d=$ doublet, $t=$ triplet, $q=$ quartet, $m=$ multiplet, $b r$ $=$ broad). Chemical shifts of the ${ }^{13} \mathrm{C}$ NMR spectra were measured relative to $\mathrm{CDCl}_{3}(77.23 \mathrm{ppm})$. Infrared spectra were recorded on a Shimadzu IR-470 spectrometer. Specific rotation data were measured on JASCO P1020 Polarimeter. HPLC was performed with an Agilent Technologies 1220 infinity LC system. Melting points were measured on Electrothermal 9100. Mass spectral data were obtained from the Korea Basic Science In-

stitute (Daegu) on a Jeol JMS 700 high resolution mass spectrometer (FAB) and Organic Chemistry Research Center in Sogang university on a Bruker Ultra High Resolution ESI Q-TOF MS / MS Compact System (ESI). 


\section{Synthesis of substrates 1,5 and 11}

1) Synthesis of substrate $\mathbf{1}$ and $\mathbf{5}$

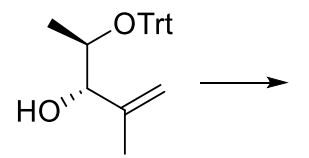

S1

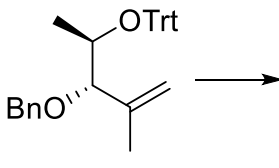

S2<smiles>C=C(C)[C@H](OBr)[C@@H](C)O</smiles>

1

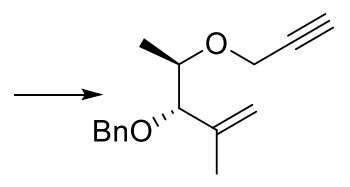

S3

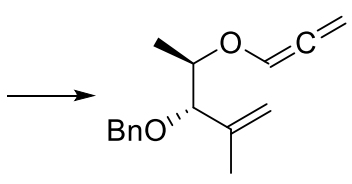

5<smiles>C=C(C)C(O)C(C)O</smiles>

(3S,4R)-2-methyl-4-(trityloxy)pent-1-en-3-ol (S1): To a solution of (R)-2-(trityloxy)propanal (7.6 g, $24.02 \mathrm{mmol})$ (synthesized according to reported procedure $\left.{ }^{[1][2]}\right)$, isopropenyl magnesium bromide solution $(0.5 \mathrm{M}$ in THF, 53 $\mathrm{mL}, 26.42 \mathrm{mmol}$ ) was added dropwise at $0^{\circ} \mathrm{C}$ under nitrogen atmosphere for over $30 \mathrm{~min}$. The reaction mixture was stirred for additional $30 \mathrm{~min}$ (monitored by TLC). The reaction mixture was quenched with saturated aqueous $\mathrm{NH}_{4} \mathrm{Cl}$ solution at $0^{\circ} \mathrm{C}$ and extracted with EtOAc $(100 \mathrm{~mL})$. The organic layer was washed with water $(2 \times 50$ $\mathrm{mL})$, dried over sodium sulfate, and concentrated under reduced pressure to give crude ( $\mathrm{dr} 2.4: 1)$ as colorless oil. The crude was purified by column chromatography on silica gel (eluted with EtOAC:Hex $=1: 25$ ) to give product $\mathbf{S 1}$ as colorless oil ( $3.75 \mathrm{~g}, 10.46 \mathrm{mmol}, 38 \%$ yield - anti isomer) and $\mathbf{S 4}$ (1.80 g, $5.04 \mathrm{mmol} 21 \%$ yield syn isomer).

Anti isomer (S1) - Rf 0.7 (EtOAc:Hex = 1:10); $[\alpha]^{23}{ }_{\mathrm{D}}-24.3\left(c\right.$ 0.97, $\left.\mathrm{CHCl}_{3}\right) ;{ }^{1} \mathrm{H} \mathrm{NMR}\left(500 \mathrm{MHz}, \mathrm{CDCl}_{3}\right) \delta 7.56(\mathrm{~d}, J=$ 7.8, 6H), 7.35-7.32 (m, 6H), 7.28-7.26 (m, 3H), $4.97(\mathrm{~s}, 1 \mathrm{H}), 4.76(\mathrm{~s}, 1 \mathrm{H}), 3.88(\mathrm{dq}, J=6.2,2.9 \mathrm{~Hz}, 1 \mathrm{H}), 3.08(\mathrm{~s}$, 1H), $2.41(\mathrm{~s}, 1 \mathrm{H}), 1.36(\mathrm{~s}, 3 \mathrm{H}), 1.05$ (d, $J=6.3 \mathrm{~Hz}, 3 \mathrm{H}) ;{ }^{13} \mathrm{C} \mathrm{NMR}\left(125 \mathrm{MHz}, \mathrm{CDCl}_{3}\right) \delta$ 144.8, 143.0, 128.9, 127.9, 127.3, 110.4, 87.5, 75.3, 71.0, 19.5, 14.1; IR (NaCl) v 3579, 2933, 1491, 1448, 1379, 1221, 1072, 1035, 984, 900 , 775, $706 \mathrm{~cm}^{-1}$; HRMS (ESI) m/z: [M + Na] $]^{+}$Calcd for $\mathrm{C}_{25} \mathrm{H}_{26} \mathrm{NaO}_{2}$ 381.1825; Found 381.1828.

Syn isomer (3R,4R)-2-methyl-4-(trityloxy)pent-1-en-3-ol (S4)- Rf 0.68 (EtOAc:Hex = 1:10); $[\alpha]^{26}$-36.5 (c 1.12, $\left.\mathrm{CH}_{2} \mathrm{Cl}_{2}\right) ;{ }^{1} \mathrm{H}$ NMR $\left(500 \mathrm{MHz}, \mathrm{CDCl}_{3}\right)$ 6 7.52-7.51 (m, 6H), 7.31-7.27 (m, 6H), 7.25-7.23 (m, 3H), $4.97(\mathrm{~s}, 1 \mathrm{H}), 4.86$ (s, 1H), 3.86 (dd, J = 6.7, $2.4 \mathrm{~Hz}, 1 \mathrm{H}), 3.40-3.35(\mathrm{~m}, 1 \mathrm{H}), 2.59$ (bs, $1 \mathrm{H}), 1.43(\mathrm{~s}, 3 \mathrm{H}), 0.87(\mathrm{~d}, J=6.2 \mathrm{~Hz}, 3 \mathrm{H}) ;{ }^{13} \mathrm{C}$ NMR $\left(125 \mathrm{MHz}, \mathrm{CDCl}_{3}\right) \delta$ 145.1, 144.5, 129.1, 127.9, 127.4, 113.9, 87.4, 79.7, 72.4, 18.2, 18.1 ; IR ( $\left.\mathrm{NaCl}\right)$ v 3559, 3086, 2978, 1491, 1447, 1222, 1055, 900, 705, $633 \mathrm{~cm}^{-1}$; HRMS (ESI) m/z: [M + Na] $]^{+}$Calcd for $\mathrm{C}_{25} \mathrm{H}_{26} \mathrm{NaO}_{2}$ 381.1825; Found 381.1827.<smiles>C=C(C)[C@@H](O[GaH2])[C@@H](C)[OH+]Cc1ccccc1</smiles> 
((((2R,3S)-3-(benzyloxy)-4-methylpent-4-en-2-yl)oxy)methanetriyl)tribenzene (S2): To a suspension of $\mathrm{NaH}$ (60\% dispersion in mineral oil, $56 \mathrm{mg}, 1.45 \mathrm{mmol})$ in DMF ( $5 \mathrm{~mL})$, solution of S1 (0.260 g, $0.725 \mathrm{mmol})$ in DMF $(2 \mathrm{~mL})$ was added dropwise at $0^{\circ} \mathrm{C}$ under inert atmosphere. The reaction mixture was stirred for $3 \mathrm{~h}$ at room temperature (monitored by TLC). After $3 \mathrm{~h}$, the mixture was quenched with water at $0^{\circ} \mathrm{C}$, and diluted with EtOAc $(20 \mathrm{~mL})$. The organic layer was washed with water $(2 \times 20 \mathrm{~mL})$, brine $(20 \mathrm{~mL})$, dried over sodium sulfate, and concentrated under reduced pressure to give crude as yellow oil. The crude was purified by column chromatography on silica gel (eluted with EtOAc:Hex $=1: 10)$ to give product $\mathbf{S 2}$ as colorless oil $(0.315 \mathrm{~g}, 0.702 \mathrm{mmol}$, $96 \%$ yield).

$\mathrm{R}_{\mathrm{f}} 0.9$ (EtOAc:Hex = 1:10); $[\alpha]^{27}{ }_{\mathrm{D}}+13.6\left(c\right.$ 1.69, $\left.\mathrm{CHCl}_{3}\right) ;{ }^{1} \mathrm{H}$ NMR $\left(300 \mathrm{MHz}, \mathrm{CDCl}_{3}\right) \delta$ 7.52-7.21 (m, 20H), 4.97 (s, 1H), $4.91(\mathrm{~s}, 1 \mathrm{H}), 4.63(\mathrm{~s}, 1 \mathrm{H}), 4.52(\mathrm{~d}, J=11.9 \mathrm{~Hz}, 1 \mathrm{H}), 4.34(\mathrm{~d}, J=11.9 \mathrm{~Hz}, 1 \mathrm{H}), 3.69(\mathrm{~m}, 1 \mathrm{H}), 3.41(\mathrm{~d}, J=3.8 \mathrm{~Hz}$, 1H), $1.33(\mathrm{~s}, 3 \mathrm{H}), 1.03$ (d, J = 6.2 Hz, 3H); $\left.{ }^{13} \mathrm{C} \mathrm{NMR} \mathrm{(125} \mathrm{MHz,} \mathrm{CDCl}_{3}\right) \delta 145.4,142.9,139.3,131.4,129.7,129.4$, $128.4,127.9,127.8,127.6,127.5,127.1,113.7,87.0,85.8,71.3,71.2,19.1,16.7 ;$ IR (NaCl) v 3061,3030, 2984, 2935, 2864, 1958, 1885, 1814, 1728, 1650, 1597, 1492, $1313 \mathrm{~cm}^{-1}$; HRMS (ESI) m/z: [M + Na] ${ }^{+}$Calcd for $\mathrm{C}_{32} \mathrm{H}_{32} \mathrm{NaO}_{2}$ 471.2295; Found 471.2296.

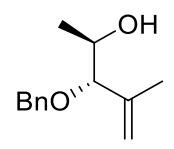

(2R,3S)-3-(benzyloxy)-4-methylpent-4-en-2-ol (1): p-TSA (10 mg, $0.071 \mathrm{mmol}$ ) was added to a solution of S2 $(0.320 \mathrm{~g}, 0.713 \mathrm{mmol})$ in $\operatorname{THF}(7 \mathrm{~mL})$ at $0^{\circ} \mathrm{C}$, and reaction mixture was stirred for $1 \mathrm{~h}$ at room temperature (monitored by TLC). After $1 \mathrm{~h}$, the mixture was quenched by adding triethylamine and concentrated under reduced pressure to obtain crude as oil. The crude was purified by column chromatography on silica gel (eluted with EtOAc:Hex $=1: 10)$ to give product 1 as colorless oil $(0.140 \mathrm{~g}, 0.719 \mathrm{mmol}, 95 \%$ yield).

$\mathrm{R}_{\mathrm{f}} 0.2$ (EtOAc:Hex = 1:10); $[\alpha]^{27}{ }_{\mathrm{D}}+68.4$ (c 1.10, $\left.\mathrm{CHCl}_{3}\right) ;{ }^{1} \mathrm{H} \mathrm{NMR}\left(300 \mathrm{MHz}, \mathrm{CDCl}_{3}\right) \delta$ 7.35-7.28 (m, 5H), $5.16(\mathrm{~s}$, 1H), $5.04(\mathrm{~s}, 1 \mathrm{H}), 4.58(\mathrm{~d}, J=11.7 \mathrm{~Hz}, 1 \mathrm{H}), 4.30(\mathrm{~d}, J=11.8 \mathrm{~Hz}, 1 \mathrm{H}), 3.86(\mathrm{dq}, J=6.2,4.4 \mathrm{~Hz}, 1 \mathrm{H}), 3.60(\mathrm{~d}, J=6.2$ $\mathrm{Hz}, 1 \mathrm{H}), 1.87(\mathrm{~d}, J=4.2 \mathrm{~Hz}, 1 \mathrm{H}), 1.78(\mathrm{~s}, 3 \mathrm{H}), 1.21(\mathrm{~d}, J=6.3 \mathrm{~Hz}, 3 \mathrm{H}) ;{ }^{13} \mathrm{C} \mathrm{NMR}\left(125 \mathrm{MHz}, \mathrm{CDCl}_{3}\right) \delta 142.5,138.5$, 128.6, 128.0, 127.9, 116.6, 87.2, 70.7, 67.9, 18.9, 18.1; IR (NaCl) v 3442, 3069, 3031, 2976, 2929, 2868, 1951, 1811, 1650, 1454, 1253, 1207, $1072 \mathrm{~cm}^{-1}$; HRMS (ESI) m/z: [M + Na] $]^{+}$Calcd for $\mathrm{C}_{13} \mathrm{H}_{18} \mathrm{NaO}_{2}$ 229.1199; Found 229.1199.

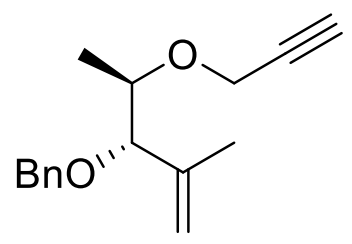


((((3S,4R)-2-methyl-4-(prop-2-yn-1-yloxy)pent-1-en-3-yl)oxy)methyl)benzene (S3): To a solution of NaH (60\% dispersion in mineral oil, $0.78 \mathrm{~g}, 19.38 \mathrm{mmol})$ in THF, solution of $\mathbf{1}(2.0 \mathrm{~g}, 9.69 \mathrm{mmol})$ in THF (total concentration, $0.3 \mathrm{M}$ ) was added at $0^{\circ} \mathrm{C}$ under nitrogen atmosphere. The reaction mixture was stirred for $30 \mathrm{~min}$ at room temperature. To this mixture propargyl bromide $\left(2.2 \mathrm{~mL}, 19.38 \mathrm{mmol}, 80 \mathrm{wt} \%\right.$ in Toluene) was added $0^{\circ} \mathrm{C}$. The resulting reaction mixture was stirred at room temperature for $24 \mathrm{~h}$. The reaction mixture was quenched with distilled water and extracted with $\mathrm{Et}_{2} \mathrm{O}(3 \times 50 \mathrm{~mL})$. The organic layer was separated, dried over $\mathrm{Na}_{2} \mathrm{SO}_{4}$, and concentrated under reduced pressure. The crude product was purifed by Flash column chromatography on silica gel (eluted with EtOAc:Hex = 1:10) to give product $\mathbf{S 3}$ as colorless oil ( $2.3 \mathrm{~g}, 9.41 \mathrm{mmol}$, 97\% yield).

$\mathrm{R}_{\mathrm{f}} 0.8$ (EtOAc:Hex = 1:10); ); $[\alpha]^{20}{ }_{\mathrm{D}}+32.9\left(\mathrm{c} 0.61, \mathrm{CH}_{2} \mathrm{Cl}_{2}\right) ;{ }^{1} \mathrm{H}$ NMR $\left(500 \mathrm{MHz}, \mathrm{CDCl}_{3}\right) \delta$ 7.34-7.25 (m, $\left.5 \mathrm{H}\right), 5.09$ (s, 1H), $5.01(\mathrm{~s}, 1 \mathrm{H}), 4.55(\mathrm{~d}, J=11.8 \mathrm{~Hz}, 1 \mathrm{H}), 4.31(\mathrm{~d}, J=11.8 \mathrm{~Hz}, 1 \mathrm{H}), 4.20-4.19(\mathrm{~m}, 2 \mathrm{H}), 3.83-3.78(\mathrm{~m}, 1 \mathrm{H}), 3.65$ (d, J=6.4 Hz, 1H), $2.38(\mathrm{t}, J=2.3 \mathrm{~Hz}, 1 \mathrm{H}), 1.79(\mathrm{~s}, 3 \mathrm{H}), 1.24(\mathrm{~d}, J=6.2 \mathrm{~Hz}, 3 \mathrm{H}) ;{ }^{13} \mathrm{C} \mathrm{NMR}\left(125 \mathrm{MHz}, \mathrm{CDCl}_{3}\right) \delta$ $142.6,138.8,128.5,127.9,127.6,115.9,86.0,80.3,74.8,74.1,70.4,56.3,18.1,16.3$; IR (NaCl) v 3293, 2923, 2857, 1453, 1374, 1070, 904, 736, 697, $625 \mathrm{~cm}^{-1}$; HRMS (ESI) m/z: [M + Na] ${ }^{+}$Calcd for $\mathrm{C}_{16} \mathrm{H}_{20} \mathrm{NaO}_{2}$ 267.1356; Found 267.1356.

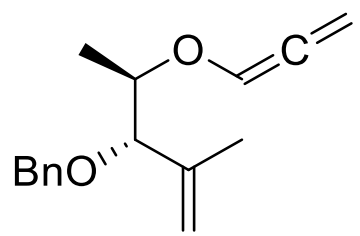

((((3S,4R)-2-methyl-4-(propa-1,2-dien-1-yloxy)pent-1-en-3-yl)oxy)methyl)benzene (5): To a solution of S3 $(1.0 \mathrm{~g}, 4.09 \mathrm{mmol})$ in THF $(12 \mathrm{~mL})$, t-BuOK $(91 \mathrm{mg}, 0.81 \mathrm{mmol})$ was added. The reaction mixture was stirred at room temperature until TLC indicated complete conversion of the starting material (approx. $1 \mathrm{~h}$ ). The resulting mixture was passed through a pad of celite and washed with $\mathrm{Et}_{2} \mathrm{O}(200 \mathrm{~mL})$. The organic layer was concentrated under reduced pressure and crude product was purified by Flash column chromatography on TEA deactivated silica gel to afford product 5 as colorless liquid $(0.73 \mathrm{~g}, 2.98 \mathrm{mmol}$, yield $73 \%)$.

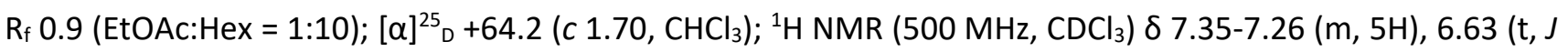
$=6.0 \mathrm{~Hz}, 1 \mathrm{H}), 5.43(\mathrm{dd}, J=8.1,6.0 \mathrm{~Hz}, 1 \mathrm{H}), 5.37(\mathrm{~d}, J=8.1,6.0 \mathrm{~Hz}, 1 \mathrm{H}), 5.08-5.07(\mathrm{~m}, 1 \mathrm{H}), 5.03(\mathrm{~s}, 1 \mathrm{H}), 4.57(\mathrm{~d}, J$ $=11.9 \mathrm{~Hz}, 1 \mathrm{H}), 4.34(\mathrm{~d}, J=11.9 \mathrm{~Hz}, 1 \mathrm{H}), 3.94-3.87(\mathrm{~m}, 1 \mathrm{H}), 3.78(\mathrm{~d}, J=6.2 \mathrm{~Hz}, 1 \mathrm{H}), 1.73(\mathrm{~s}, 3 \mathrm{H}), 1.26(\mathrm{~d}, J=6.1$ $\mathrm{Hz}, 3 \mathrm{H}) ;{ }^{13} \mathrm{C}$ NMR $\left(125 \mathrm{MHz}, \mathrm{CDCl}_{3}\right) \delta 201.5,142.3,138.7,128.4,127.9,127.6,120.7,115.5,90.6,84.9,74.8$, 70.7, 18.3, 16.1; IR (NaCl) v 3068, 3033, 2975, 2932, 2867, 1952, 1652, 1497, 1352, $1202 \mathrm{~cm}^{-1}$; HRMS (ESI) m/z: $[\mathrm{M}+\mathrm{Na}]^{+}$Calcd for $\mathrm{C}_{16} \mathrm{H}_{20} \mathrm{NaO}_{2}$ 267.1356; Found 267.1359. 
2) Synthesis of substrate 11

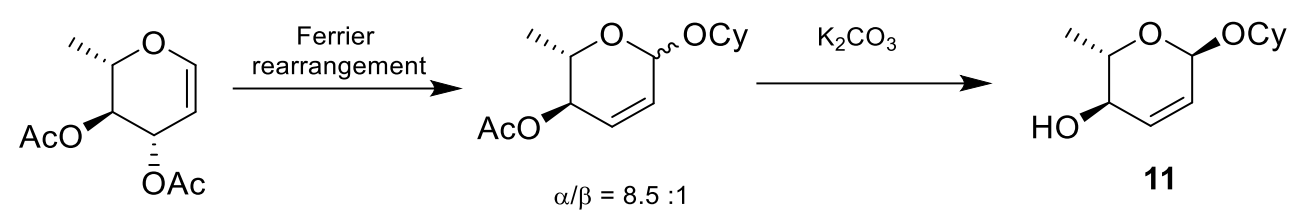

(2S,3R,6R)-6-(cyclohexyloxy)-2-methyl-3,6-dihydro-2H-pyran-3-ol (11): The alcohol 11 (600 mg, $2.83 \mathrm{mmol})$ was synthesized from 3,4-diacetyl glucal ( $1.0 \mathrm{~g}, 4.86 \mathrm{mmol})$ in $58 \%$ yield over two steps as per reported procedure ${ }^{3}$.

m.p. 43-48 ${ }^{\circ} \mathrm{C} ; \mathrm{R}_{\mathrm{f}} 0.2$ (EtOAc:Hex = 1:10); $[\alpha]^{23} \mathrm{D}-57.6\left(c 0.31, \mathrm{CH}_{2} \mathrm{Cl}_{2}\right) ;{ }^{1} \mathrm{H} \mathrm{NMR}\left(500 \mathrm{MHz}, \mathrm{CDCl}_{3}\right) \delta$ 7.38-7.27 (m, $5 \mathrm{H}), 5.86(\mathrm{ddd}, J=17.3,10.4,5.7 \mathrm{~Hz}, 1 \mathrm{H}), 5.36(\mathrm{~d}, J=17.3 \mathrm{~Hz}, 1 \mathrm{H}), 5.23(\mathrm{~d}, J=10.5 \mathrm{~Hz}, 1 \mathrm{H}), 5.10(\mathrm{~s}, 1 \mathrm{H}), 5.06(\mathrm{~s}$, $1 \mathrm{H}), 5.01(\mathrm{~d}, J=5.7 \mathrm{~Hz}, 1 \mathrm{H}), 4.59(\mathrm{~d}, J=11.9 \mathrm{~Hz}, 1 \mathrm{H}), 4.34(\mathrm{~d}, J=11.9 \mathrm{~Hz}, 1 \mathrm{H}), 3.89-3.84(\mathrm{~m}, 1 \mathrm{H}), 3.72(\mathrm{~d}, J=6.2$ $\mathrm{Hz}, 1 \mathrm{H}), 3.65-3.59(\mathrm{~m}, 1 \mathrm{H}), 1.94-1.89(\mathrm{~m}, 2 \mathrm{H}), 1.82(\mathrm{~s}, 3 \mathrm{H}), 1.78-1.72(\mathrm{~m}, 2 \mathrm{H}), 1.54-1.18(\mathrm{~m}, 9 \mathrm{H}) ;{ }^{13} \mathrm{C}$ NMR $(125$ $\mathrm{MHz}, \mathrm{CDCl}_{3}$ ) $\delta 133.3,133.2,127.7,127.7,92.8,76.6,70.1,70.0,68.1,34.2,32.5,25.8,24.6,24.5,18.2$; IR (NaCl) v 3450, 2932, 2857, 1450, 1376, 1099, 1049, 1003, 886, $734 \mathrm{~cm}^{-1}$; HRMS (ESI) m/z: [M + Na] Calcd for $\mathrm{C}_{12} \mathrm{H}_{20} \mathrm{NaO}_{3}$ 235.1304; Found 235.1311.

\section{Synthesis of cyclic 0,0 -acetal $7 \mathrm{a}$ and $7 \mathrm{~b}$}

1) General procedure of Pd-ctalyzed hydroalkoxylation

To a solution of $\mathrm{Pd}_{2} \mathrm{dba}_{3}(2.5 \mathrm{~mol} \% / 3.5 \mathrm{~mol} \% / 5 \mathrm{~mol} \%)$ and $(S, S)-\mathrm{L}$ or $(R, R)-\mathrm{L}(5 \mathrm{~mol} \%$ or $7 \mathrm{~mol} \% 10 \mathrm{~mol} \%)$ in toluene, a solution of starting material allene (1 equiv), alcohol ( 2 equiv), and triethylamine ( 0.1 equiv) in toluene (total concentration 0.5 M) was added. The reaction mixture was heated at $40{ }^{\circ} \mathrm{C}$ until TLC indicated complete conversion of starting material. The crude was directly purified by column chromatography on silica gel (deactivated by triethylamine) to give desired product. Silica gel was deactivated with few drops of $\mathrm{Et}_{3} \mathrm{~N}$ and $\mathrm{CDCl}_{3}$ was deactivated with basic alumina before use.

2) General procedure of RCM (ring-closing metathesis)

To a solution of acyclic $O, O$-acetal (1 equiv) in $\mathrm{CH}_{2} \mathrm{Cl}_{2}(0.1 \mathrm{M})$ Hoveyda Grubbs' $2^{\text {nd }}$ catalyst was added (5 mol\%). The reaction mixture was stirred until TLC indicated complete conversion of the starting material. The solvent was removed under reduced pressure and then the crude product was isolated by Flash column chromatography on deactivated silica gel. $\mathrm{CDCl}_{3}$ was deactivated with basic alumina before use. 
3) Synthesis of $7 a$ from 5 and 6

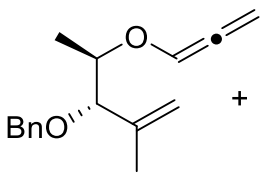

5

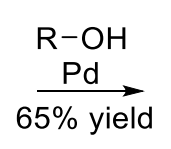

6

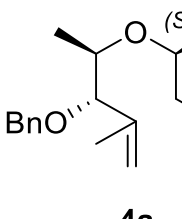

$4 a$

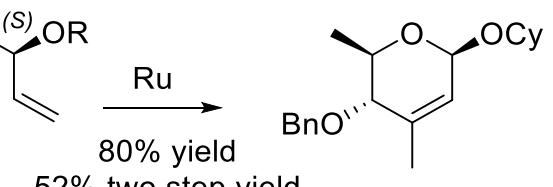
$52 \%$ two step yield

$7 a$<smiles>C=C1C(=C)[C@@H](O[Ga])[C@@H](C)O[C@H]1OC</smiles>

((((3S,4R)-4-(((R)-1-(cyclohexyloxy)allyl)oxy)-2-methylpent-1-en-3-yl)oxy)methyl)benzene (4a): Following the general procedure, to a solution of $\mathrm{Pd}_{2} \mathrm{dba}_{3}(46.0 \mathrm{mg}, 0.05 \mathrm{mmol})$ and $(S, S)-3(69.1 \mathrm{mg}, 0.10 \mathrm{mmol})$ in toluene, a solution of $5(244 \mathrm{mg}, 1.00 \mathrm{mmol}), 6(200 \mathrm{mg}, 0.20 \mathrm{mmol})$, and triethylamine $(14 \mu \mathrm{L}, 0.1 \mathrm{mmol})$ in toluene (total concentration $0.5 \mathrm{M}$ ) was added. The reaction mixture was heated at $40{ }^{\circ} \mathrm{C}$ using an oil bath for $24 \mathrm{~h}$ (monitored by TLC). The crude was directly purified by flash column chromatography (eluent: hexane/EtOAc 90:10) on silica gel to afford $223 \mathrm{mg}(0.65 \mathrm{mmol}, 65 \%)$ of acyclic 0,0 -acetal 4 a as colorless oil.

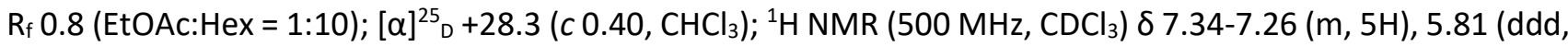
$J=17.3,10.5,5.6 \mathrm{~Hz}, 1 \mathrm{H}), 5.31(\mathrm{~d}, J=16.2 \mathrm{~Hz}, 1 \mathrm{H}), 5.18(\mathrm{~d}, J=10.5 \mathrm{~Hz}, 1 \mathrm{H}), 5.07(\mathrm{~s}, 1 \mathrm{H}), 5.03(\mathrm{~s}, 1 \mathrm{H}), 4.99(\mathrm{~d}, J$ $=5.4 \mathrm{~Hz}, 1 \mathrm{H}), 4.56(\mathrm{~d}, J=11.9 \mathrm{~Hz}, 1 \mathrm{H}), 4.32(\mathrm{~d}, J=11.9 \mathrm{~Hz}, 1 \mathrm{H}), 3.87-3.82(\mathrm{~m}, 1 \mathrm{H}), 3.68(\mathrm{~d}, J=5.7 \mathrm{~Hz}, 1 \mathrm{H}), 3.50-$ $3.46(\mathrm{~m}, 1 \mathrm{H}), 1.88-1.82(\mathrm{~m}, 2 \mathrm{H}), 1.76(\mathrm{~s}, 3 \mathrm{H}), 1.73-1.72(\mathrm{~m}, 2 \mathrm{H}), 1.52-1.18(\mathrm{~m}, 9 \mathrm{H}) ;{ }^{13} \mathrm{C} \mathrm{NMR}\left(125 \mathrm{MHz}, \mathrm{CDCl}_{3}\right) \delta$ 142.6, 138.9, 136.9, 128.5, 127.9, 127.6, 117.3, 115.5, 101.0, 86.1, 74.2, 73.3, 70.6, 33.4, 33.2, 25.9, 24.5, 24.4, 18.9, 18.0; IR (NaCl) v 3030, 2932, 2857, 1734, 1650, 1543, 1496, 1372, 1178, $1024 \mathrm{~cm}^{-1}$; HRMS (ESI) m/z: [M + $\mathrm{Na}]^{+}$Calcd for $\mathrm{C}_{22} \mathrm{H}_{32} \mathrm{NaO}_{3}$ 367.2244; Found 367.2245.<smiles>CC1=C[C@@H](O[AlH2])O[C@H](C)[C@@H]1Br</smiles>

(2R,3S,6R)-3-(benzyloxy)-6-(cyclohexyloxy)-2,4-dimethyl-3,6-dihydro-2H-pyran (7a): Following the general procedure, 4a (210 mg, $0.610 \mathrm{mmol})$ was reacted with the Hoveyda Grubbs' $2^{\text {nd }}$ catalyst $(19 \mathrm{mg}, 0.0305 \mathrm{mmol})$ in $\mathrm{CH}_{2} \mathrm{Cl}_{2}(6 \mathrm{~mL})$ at $40{ }^{\circ} \mathrm{C}$ using an oil bath for $24 \mathrm{~h}$ (monitored by TLC). Flash column chromatography on silica gel (eluted with Hexane:EtOAc = 95:5) afforded $7 \mathrm{a}$ as a colorless oil $(155 \mathrm{mg}, 0.487 \mathrm{mmol}, 80 \%$ yield; 52\% twostep yield of major diastereomer 7 a from allene 5$)$. The diastereomeric ratio (8:1) was determined by integration of the RCM crude mixture.

$R_{\mathrm{f}} 0.70$ (EtOAc:Hex 1:10); $[\alpha]^{25}{ }_{\mathrm{D}}+17.3\left(\mathrm{c} 0.80, \mathrm{CHCl}_{3}\right) ;{ }^{1} \mathrm{H} \mathrm{NMR}\left(500 \mathrm{MHz}, \mathrm{CDCl}_{3}\right) \delta$ 7.35-7.28 (m, $\left.5 \mathrm{H}\right), 5.55$ (d, J $=1.1 \mathrm{~Hz}, 1 \mathrm{H}), 5.50(\mathrm{~d}, J=1.5 \mathrm{~Hz}, 1 \mathrm{H}), 4.60(\mathrm{~s}, 2 \mathrm{H}), 3.95-3.90(\mathrm{~m}, 1 \mathrm{H}), 3.72-3.66(\mathrm{~m}, 1 \mathrm{H}), 3.62(\mathrm{~d}, J=5.5 \mathrm{~Hz}, 1 \mathrm{H})$, 1.98-1.93 (m, 2H), $1.80(\mathrm{~s}, 3 \mathrm{H}), 1.76-1.74(\mathrm{~m}, 2 \mathrm{H}), 1.59-1.17(\mathrm{~m}, 9 \mathrm{H}) ;{ }^{13} \mathrm{C} \mathrm{NMR}\left(125 \mathrm{MHz}, \mathrm{CDCl}_{3}\right) \delta 138.4,136.9$, 
128.6, 128.2, 128.0, 125.7, 94.2, 78.7, 75.8, 72.7, 71.5, 34.0, 32.2, 25.9, 24.6, 24.4, 19.8, 19.7; IR (NaCl) v 3032, 2932, 2857, 1740, 1497, 1354, 1260, 1158, 1083, $1014 \mathrm{~cm}^{-1}$; HRMS (ESI) m/z: [M + Na] Calcd for $\mathrm{C}_{20} \mathrm{H}_{28} \mathrm{NaO}_{3}$ 339.1931; Found 339.1932.

4) Alternative method for the Synthesis of $\mathbf{4 a}$ from alcohol $\mathbf{1}$ and allene $\mathbf{2}$

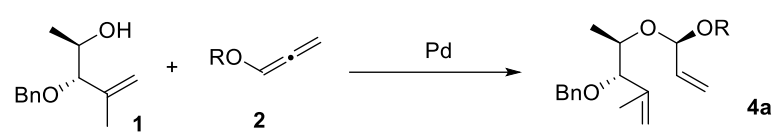

Following the general procedure, to a solution of $\mathrm{Pd}_{2} \mathrm{dba}_{3}(3.3 \mathrm{mg}, 3.64 \mu \mathrm{mol})$ and $(\boldsymbol{R}, \boldsymbol{R})-\mathbf{3}(5.0 \mathrm{mg}, 7.27 \mu \mathrm{mol})$ in toluene, a solution of $2(20 \mathrm{mg}, 0.145 \mathrm{mmol}), 1(30 \mathrm{mg}, 0.145 \mathrm{mmol})$, and triethylamine $(2 \mu \mathrm{L}, 14.5 \mu \mathrm{mol})$ in toluene (total concentration $0.5 \mathrm{M}$ ) was added. The reaction mixture was heated at $40{ }^{\circ} \mathrm{C}$ using an oil bath for $24 \mathrm{~h}$ (monitored by TLC). The crude product was directly purified by column chromatography (eluent: hexane/EtOAc 95:5) on silica gel to afford $10 \mathrm{mg}(0.029 \mathrm{mmol}, 20 \%)$ of acyclic 0,0 -acetal 4 a.

5) Synthesis of $7 b$
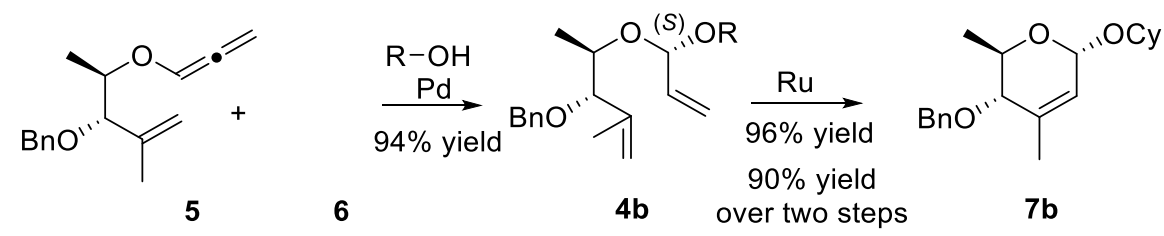<smiles>C=C1C(=C)[C@@H](Br)[C@@H](C)O[C@@H]1O[Ga]</smiles>

((((3S,4R)-4-(((S)-1-(cyclohexyloxy)allyl)oxy)-2-methylpent-1-en-3-yl)oxy)methyl)benzene (4b): Following the general procedure, to a solution of $\mathrm{Pd}_{2} \mathrm{dba}_{3}(10.6 \mathrm{mg}, 11.57 \mu \mathrm{mol})$ and $(\boldsymbol{R}, \boldsymbol{R})-3(16.0 \mathrm{mg}, 23.16 \mu \mathrm{mol})$ in toluene, a solution of $5(81 \mathrm{mg}, 0.33 \mathrm{mmol}), 6(66 \mathrm{mg}, 0.66 \mathrm{mmol})$, and triethylamine $(4.6 \mu \mathrm{L}, 33.0 \mu \mathrm{mol})$ in toluene (total concentration $0.5 \mathrm{M}$ ) was added. The reaction mixture was heated at $40{ }^{\circ} \mathrm{C}$ using an oil bath for $2 \mathrm{~h}$ (monitored by TLC). The crude was directly purified by flash column chromatography (eluent: hexane/EtOAc 90:10) on silica gel to afford acyclic $O, O$-acetal 4 b as colorless oil ( $107 \mathrm{mg}, 0.31 \mathrm{mmol}, 94 \%$ ).

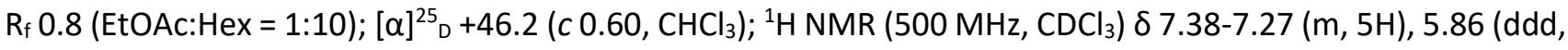
$J=17.3,10.4,5.7 \mathrm{~Hz}, 1 \mathrm{H}), 5.36(\mathrm{~d}, J=17.3 \mathrm{~Hz}, 1 \mathrm{H}), 5.23(\mathrm{~d}, J=10.5 \mathrm{~Hz}, 1 \mathrm{H}), 5.10(\mathrm{~s}, 1 \mathrm{H}), 5.06(\mathrm{~s}, 1 \mathrm{H}), 5.01(\mathrm{~d}, J$ $=5.7 \mathrm{~Hz}, 1 \mathrm{H}), 4.59(\mathrm{~d}, J=11.9 \mathrm{~Hz}, 1 \mathrm{H}), 4.34(\mathrm{~d}, J=11.9 \mathrm{~Hz}, 1 \mathrm{H}), 3.89-3.84(\mathrm{~m}, 1 \mathrm{H}), 3.72(\mathrm{~d}, J=6.2 \mathrm{~Hz}, 1 \mathrm{H}), 3.65-$ $3.59(\mathrm{~m}, 1 \mathrm{H}), 1.94-1.89(\mathrm{~m}, 2 \mathrm{H}), 1.82(\mathrm{~s}, 3 \mathrm{H}), 1.78-1.72(\mathrm{~m}, 2 \mathrm{H}), 1.54-1.18(\mathrm{~m}, 9 \mathrm{H}) ;{ }^{13} \mathrm{C} \mathrm{NMR}\left(125 \mathrm{MHz}, \mathrm{CDCl}_{3}\right) \delta$ 142.7, 138.9, 137.2, 128.4, 127.9, 127.5, 117.4, 115.5, 99.7, 86.3, 74.2, 71.3, 70.6, 33.4, 32.6, 25.9, 24.5, 24.3, 18.5, 17.1 IR (NaCl) v 3069, 3030, 2933, 2857, 1651, 1497, 1373, 1207, 1068, $1026 \mathrm{~cm}^{-1}$; HRMS (ESI) m/z: [M + $\mathrm{Na}]^{+}$Calcd for $\mathrm{C}_{22} \mathrm{H}_{32} \mathrm{NaO}_{3}$ 367.2244; Found 367.2245. 


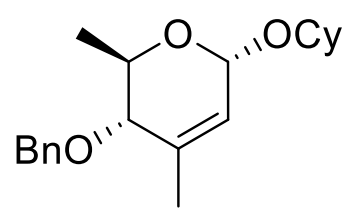

(2R,3S,6S)-3-(benzyloxy)-6-(cyclohexyloxy)-2,4-dimethyl-3,6-dihydro-2H-pyran (7b): Following the general procedure, 4b (94 mg, $0.273 \mathrm{mmol}$ ) was reacted with the Hoveyda Grubbs' $2^{\text {nd }}$ catalyst $(8.5 \mathrm{mg}, 13.6 \mu \mathrm{mol})$ in $\mathrm{CH}_{2} \mathrm{Cl}_{2}(2.7 \mathrm{~mL})$ at $40{ }^{\circ} \mathrm{C}$ using an oil bath for overnight (monitored by TLC). Flash column chromatography on silica gel (eluted with Hexane:EtOAc = 95:5) afforded 7b as a colorless oil ( $82.4 \mathrm{mg}, 0.260 \mathrm{mmol}, 96 \%$ yield, 90\% two-step yield of $\mathbf{7 b}$ from allene $\mathbf{5})$. The diastereomeric ratio $(<1: 25)$ was determined by crude NMR.

$R_{\mathrm{f}} 0.70$ (EtOAc:Hex 1:10); $[\alpha]^{25}{ }_{\mathrm{D}}+86.8$ (c 0.70, $\left.\mathrm{CHCl}_{3}\right) ;{ }^{1} \mathrm{H}$ NMR $\left(500 \mathrm{MHz}, \mathrm{CDCl}_{3}\right) \delta$ 7.35-7.28 (m, 5H), 5.50 (s, 1H), $5.04(\mathrm{~s}, 1 \mathrm{H}), 4.65(\mathrm{~d}, J=11.3 \mathrm{~Hz}, 1 \mathrm{H}), 4.57(\mathrm{~d}, J=11.2 \mathrm{~Hz}, 1 \mathrm{H}), 4.08(\mathrm{dq}, J=9.1,6.2 \mathrm{~Hz}, 1 \mathrm{H}), 3.67(\mathrm{~d}, J=9.1$ $\mathrm{Hz}, 1 \mathrm{H}), 3.62-3.56(\mathrm{~m}, 1 \mathrm{H}), 1.92-1.89(\mathrm{~m}, 2 \mathrm{H}), 1.79(\mathrm{~s}, 3 \mathrm{H}), 1.78-1.72(\mathrm{~m}, 2 \mathrm{H}), 1.56-1.19(\mathrm{~m}, 9 \mathrm{H}) ;{ }^{13} \mathrm{C}$ NMR $(125$ $\left.\mathrm{MHz}, \mathrm{CDCl}_{3}\right) \delta 139.8,138.6,128.6,127.9$ 127.8, 123.7, 93.4, 80.2, 76.1, 72.9, 65.6, 34.3, 32.6, 25.9, 24.8, 24.6, 18.8, 18.7; IR (NaCl) v 2932, 2856, 1451, 1390, 1137, 1056, 1021, 958, 735, $698 \mathrm{~cm}^{-1} ; \mathrm{HRMS}(\mathrm{ESI}) \mathrm{m} / \mathrm{z}:[\mathrm{M}+\mathrm{Na}]^{+}$ Calcd for $\mathrm{C}_{20} \mathrm{H}_{28} \mathrm{NaO}_{3}$ 339.1931; Found 339.1934.

\section{Synthesis of C3-methyl amino sugar - D-saccharosamine cyclohexyl glycoside 10}<smiles>CC1=C[C@@H](O[AlH2])O[C@@H](C)[C@@H]1O</smiles>

(2R,3S,6R)-6-(cyclohexyloxy)-2,4-dimethyl-3,6-dihydro-2H-pyran-3-ol (8): In a two-neck flame-dried round bottom flask, fitted with Dewar flask (cooled to $-78^{\circ} \mathrm{C}$ ), $\mathrm{NH}_{3}\left(24 \mathrm{~mL}\right.$ ) was trapped at $-78^{\circ} \mathrm{C}$. To this mixture, $\mathrm{Na}$ metal $(60 \mathrm{mg}, 2.61 \mathrm{mmol}$ ) was added, followed by the addition of solution of 7a (165 mg, $0.52 \mathrm{mmol})$ in THF $(9.0 \mathrm{~mL})$. The reaction mixture was stirred at $-78{ }^{\circ} \mathrm{C}$ for $1 \mathrm{~h}$ (monitored by TLC) and allowed to warm to room temperature. The reaction mixture was quenched with solid $\mathrm{NH}_{4} \mathrm{Cl}$ until blue color disappeared, and was extracted with EtOAc $(3 \times 20 \mathrm{~mL})$. The combined organic layers was dried over $\mathrm{Na}_{2} \mathrm{SO}_{4}$ and concentrated under reduced pressure to give crude. The Flash column chromatography of crude on silica gel (eluted with EtOAc:Hex = 5:5) afforded 8 as white solid (110 mg, $0.486 \mathrm{mmol}, 93 \%)$.

m.p. $62-64^{\circ} \mathrm{C} ; R_{\mathrm{f}} 0.60$ (EtOAc:Hex 5:5); $[\alpha]^{29}{ }_{\mathrm{D}}-16.4\left(\mathrm{c} 0.52, \mathrm{CHCl}_{3}\right) ;{ }^{1} \mathrm{H} \mathrm{NMR}\left(500 \mathrm{MHz}, \mathrm{CDCl}_{3}\right) \delta 5.49(\mathrm{~s}, 1 \mathrm{H}), 5.16$ $(\mathrm{d}, J=1.25 \mathrm{~Hz}, 1 \mathrm{H}), 3.72-3.65(\mathrm{~m}, 3 \mathrm{H}), 1.97-1.94(\mathrm{~m}, 2 \mathrm{H}), 1.81(\mathrm{~s}, 3 \mathrm{H}), 1.76-1.69(\mathrm{~m}, 3 \mathrm{H}), 1.55-1.52(\mathrm{~m}, 1 \mathrm{H})$, 1.40-1.13 (m, 8H); $\left.{ }^{13} \mathrm{C} \mathrm{NMR} \mathrm{(125} \mathrm{MHz,} \mathrm{CDCl}_{3}\right) \delta$ 138.1, 124.6, 94.1, 75.8, 73.7, 71.4, 33.8, 32.0, 25.7, 24.4, 24.1, 19.1, 18.8; IR (NaCl) v 3420, 2933, 2857, 1450, 1357, 1258, 1192, 1075, 961, 922, 892, $854 \mathrm{~cm}^{-1}$; HRMS (ESI) $\mathrm{m} / \mathrm{z}:[\mathrm{M}+\mathrm{Na}]^{+}$Calcd for $\mathrm{C}_{13} \mathrm{H}_{22} \mathrm{NaO}_{3}$ 249.1461; Found 249.1462 . 


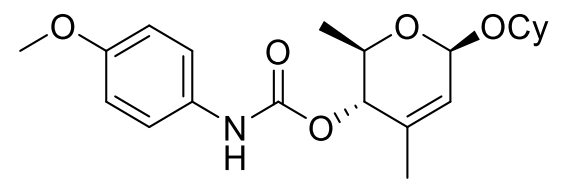

((2R,3S,6R)-6-(cyclohexyloxy)-2,4-dimethyl-3,6-dihydro-2H-pyran-3-yl (4-methoxyphenyl)carbamate (9): To a solution of $8(120 \mathrm{mg}, 0.530 \mathrm{mmol})$ in $\mathrm{CH}_{2} \mathrm{Cl}_{2}(1.5 \mathrm{~mL}), p$-methoxyphenyl isocyanate $(78 \mu \mathrm{L}, 0.583 \mathrm{mmol})$ and $\mathrm{DBU}(8.0 \mu \mathrm{L}, 61.9 \mu \mathrm{mol})$ were added at room temperature. After stirring reaction mixture for $4 \mathrm{~h}$ (monitored by $T(C)$, the mixture was concentrated under reduced pressure. The column chromatography of crude on silica gel (eluted with EtOAc:Hex = 1:10) afforded 9 as white solid (179 mg, $0.480 \mathrm{mmol}, 90 \%$ ).

m.p. $125-131^{\circ} \mathrm{C}{ }^{\circ} \mathrm{C} ; \operatorname{Rf} 0.3$ (EtOAc:Hex 1:10); $[\alpha]^{27}{ }_{D}+32.6\left(c 0.26, \mathrm{CH}_{2} \mathrm{Cl}_{2}\right) ; 1 \mathrm{H} \mathrm{NMR}(500 \mathrm{MHz}, \mathrm{CDCl}) \delta$ 7.30-7.28 $(\mathrm{m}, 2 \mathrm{H}), 6.88-6.83(\mathrm{~m}, 2 \mathrm{H}), 6.53(\mathrm{bs}, 1 \mathrm{H}), 5.65(\mathrm{~s}, 1 \mathrm{H}), 5.22(\mathrm{~s}, 1 \mathrm{H}), 5.04(\mathrm{~d}, J=4.2 \mathrm{~Hz}, 1 \mathrm{H}), 3.96-3.91(\mathrm{~m}, 1 \mathrm{H})$, $3.79(\mathrm{~s}, 3 \mathrm{H}), 3.74-3.69(\mathrm{~m}, 1 \mathrm{H}), 1.98-1.94(\mathrm{~m}, 2 \mathrm{H}), 1.78(\mathrm{~s}, 3 \mathrm{H}), 1.76-1.73(\mathrm{~m}, 2 \mathrm{H}), 1.55-1.52(\mathrm{~m}, 1 \mathrm{H}), 1.41-1.15$ (m, 8H); ${ }^{13} \mathrm{C}$ NMR $(125 \mathrm{MHz}, \mathrm{CDCl} 3) \delta 156.3,153.8,134.5,130.9,126.5,120.7,114.5,93.7,75.8,72.7,71.5$, 55.7, 33.9, 32.1, 25.9, 24.6, 24.4, 19.5, 19.2; IR (NaCl) 3319, 2933, 2856, 1703, 1535, 1517, 1225, 1084, 1056, 828 v cm-1; HRMS (ESI) m/z: [M + Na] $]^{+}$Calcd for $\mathrm{C}_{21} \mathrm{H}_{29} \mathrm{NNaO}_{5}$ 398.1938; Found 398.1937.

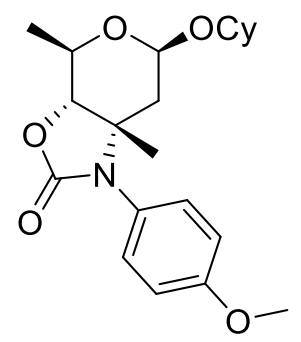

((3aS,4R,6R,7aS)-6-(cyclohexyloxy)-1-(4-methoxyphenyl)-4,7a-dimethylhexahydro-2H-pyrano[4,3-d]oxazol2-one (10): To a solution of 9 (38 mg, $0.101 \mathrm{mmol})$ in THF:DMSO (10:1) (5 mL) in a seal-tube, $\mathrm{NaHCO}_{3}(102 \mathrm{mg}$, $1.21 \mathrm{mmol}$ ) and 2-iodoxybenzoic acid, IBX (170 mg, $0.607 \mathrm{mmol})$ were added, and reaction mixture was heated at $90{ }^{\circ} \mathrm{C}$ for $12 \mathrm{~h}$ (monitored by TLC). The mixture was allowed to cool to room temperature and was diluted with EtOAc $(10 \mathrm{~mL})$. The organic layer was washed with $5 \% \mathrm{NaHCO}_{3}(3 \times 10 \mathrm{~mL})$, dried over $\mathrm{Na}_{2} \mathrm{SO}_{4}$, and concentrated under reduced pressure. The column chromatography of crude on silica gel (eluted with EtOAc:Hex $=1: 10$ ) afforded 10 as yellow oil (23.3 mg, $0.062 \mathrm{mmol}, 61 \%)$.

$R_{\mathrm{f}} 0.4$ (EtOAc:Hex 3:10); $[\alpha]^{26}{ }_{\mathrm{D}}+24.2\left(\mathrm{c} 0.55, \mathrm{CH}_{2} \mathrm{Cl}_{2}\right) ;{ }^{1} \mathrm{H}$ NMR $\left(500 \mathrm{MHz}, \mathrm{CDCl}_{3}\right)$ ठ 7.11-7.08 (m, 2H), 6.97-6.94 $(\mathrm{m}, 2 \mathrm{H}), 4.91(\mathrm{dd}, J=8.7,2.3 \mathrm{~Hz}, 1 \mathrm{H}), 3.88(\mathrm{~d}, J=8.9 \mathrm{~Hz}, 1 \mathrm{H}), 3.82(\mathrm{~s}, 3 \mathrm{H}), 3.73-3.67(\mathrm{~m}, 2 \mathrm{H}), 2.00(\mathrm{dd}, J=$ 15.0, 2.3 Hz, 1H), 1.92-1.86 (m, 2H), 1.75- $1.72(\mathrm{~m}, 2 \mathrm{H}), 1.62(\mathrm{dd}, J=14.9,8.8 \mathrm{~Hz}, 1 \mathrm{H}), 1.54(\mathrm{~m}, 1 \mathrm{H}), 1.43-$ 1.16, $\mathrm{m}, 11 \mathrm{H}) ;{ }^{13} \mathrm{C}$ NMR $\left(125 \mathrm{MHz}, \mathrm{CDCl}_{3}\right) \delta 159.7,157.1,130.4,127.1,115.1,96.1,81.1,76.3,70.8,62.6$, 55.7, 38.0, 33.8, 31.9, 26.8, 25.8, 24.3, 24.1, 19.1; IR (NaCl) v 2933, 2856, 1760, 1514, 1369, 1250, 1092, 1072, 1034, $833 \mathrm{~cm}^{-1}$; HRMS (ESI) m/z: [M + Na] ${ }^{+}$Calcd for $\mathrm{C}_{21} \mathrm{H}_{29} \mathrm{NNaO}_{5} 398.1938$; Found 398.1940. 


\section{Synthesis of 13a and 13b via Pd-catalyzed hydroalkoxylation followed by Ring-Closing Metathesis}

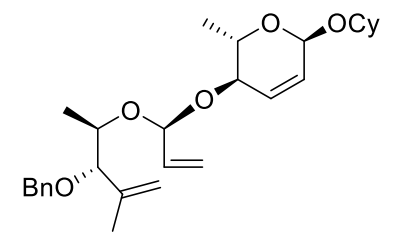

(2S,3R,6R)-3-(((S)-1-(((2R,3S)-3-(benzyloxy)-4-methylpent-4-en-2-yl)oxy)allyl)oxy)-6-(cyclohexyloxy)-2-methyl-3,6-dihydro-2H-pyran (12a): Following the general procedure, to a solution of $\mathrm{Pd}_{2} \mathrm{dba}_{3}$ (32.8 $\mathrm{mg}, 35.8$ $\mu \mathrm{mol})$ and $(S, S)-\mathbf{L}(49.5 \mathrm{mg}, 71.6 \mu \mathrm{mol})$ in toluene, a solution of $5(250 \mathrm{mg}, 1.02 \mathrm{mmol})$, alcohol $11(435 \mathrm{mg}$, $2.05 \mathrm{mmol})$, and triethylamine $(14 \mu \mathrm{L}, 0.10 \mathrm{mmol})$ in toluene (total concentration $0.5 \mathrm{M}$ ) was added. The reaction mixture was heated at $40^{\circ} \mathrm{C}$ using an oil bath for $1 \mathrm{~h}$ (monitored by TLC). The crude was directly purified by flash column chromatography (eluent: hexane/EtOAc 95:5) on silica gel (deactivated by triethylamine) to give acyclic 0,0 -acetal $12 \mathrm{a}$ as colorless oil $(460 \mathrm{mg}, 1.01 \mathrm{mmol}, 99 \%)$. The diastereomeric ratio of the crude was calculated as $>25: 1$ by ${ }^{1} \mathrm{H}$ NMR spectroscopy of the crude mixture.

$R_{\mathrm{f}} 0.70$ (EtOAc:Hex 1:10); $[\alpha]^{23}{ }_{\mathrm{D}}-27.6\left(\mathrm{c} 0.81, \mathrm{CHCl}_{3}\right) ;{ }^{1} \mathrm{H}$ NMR $\left(500 \mathrm{MHz}, \mathrm{CDCl}_{3}\right) \delta$ 7.34-7.24 (m, 5H), $5.96(\mathrm{~d}, J$ $=10.3 \mathrm{~Hz}, 1 \mathrm{H}$ ), 5.77 (ddd, $J=16.1,10.6,5.6 \mathrm{~Hz}, 1 \mathrm{H}), 5.66$ (ddd, $J=10.2,2.5,1.9,1 \mathrm{H}), 5.33(\mathrm{~d}, J=17.3 \mathrm{~Hz}, 1 \mathrm{H}$ ), $5.23(\mathrm{~d}, J=10.4 \mathrm{~Hz}, 1 \mathrm{H}), 5.06-5.04(\mathrm{~m}, 3 \mathrm{H}), 5.0(\mathrm{~s}, 1 \mathrm{H}), 4.54(\mathrm{~d}, J=11.9 \mathrm{~Hz}, 1 \mathrm{H}), 4.30(\mathrm{~d}, J=11.9 \mathrm{~Hz}, 1 \mathrm{H}), 3.94-$ $3.86(\mathrm{~m}, 3 \mathrm{H}), 3.65(\mathrm{~d}, J=5.7 \mathrm{~Hz}, 1 \mathrm{H}), 3.62-3.57(\mathrm{~m}, 1 \mathrm{H}), 1.88-1.87(\mathrm{~m}, 2 \mathrm{H}), 1.75-1.73(\mathrm{~m}, 5 \mathrm{H}), 1.53-1.52(\mathrm{~m}, 1 \mathrm{H})$, 1.42-1.13 (m, 11H); ${ }^{13} \mathrm{C}$ NMR $\left(125 \mathrm{MHz}, \mathrm{CDCl}_{3}\right) \delta 142.1,138.6,136.0,131.7,128.3,127.7,127.4,126.9,118.2$, 115.6, 101.7, 92.3, 85.8, 76.0, 73.9, 72.9, 70.4, 65.7, 33.9, 32.2, 25.6, 24.4, 24.2, 18.6, 18.3, 17.5; IR (NaCl) v 2972, 2932, 2857, 1497, 1374, 1299, 110, 1049, 903, 825, 734, $698 \mathrm{~cm}^{-1}$; HRMS (ESI) m/z: [M + Na] Calcd for $\mathrm{C}_{28} \mathrm{H}_{40} \mathrm{NaO}_{5} 479.2768$; Found 479.2771.

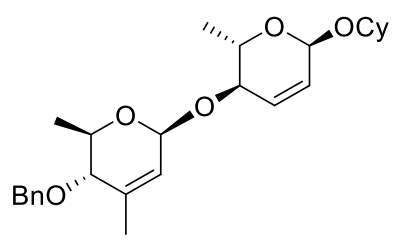

(2R,3S,6S)-3-(benzyloxy)-6-(((2S,3R,6R)-6-(cyclohexyloxy)-2-methyl-3,6-dihydro-2H-pyran-3-yl)oxy)-2,4-dimethyl-3,6-dihydro-2H-pyran (13a): Following the general procedure, 12a (420 mg, $0.919 \mathrm{mmol}$ ) was reacted with the Hoveyda Grubbs' $2^{\text {nd }}$ catalyst $(28.8 \mathrm{mg}, 46.0 \mu \mathrm{mol})$ in $\mathrm{CH}_{2} \mathrm{Cl}_{2}(10 \mathrm{~mL})$ at $40{ }^{\circ} \mathrm{C}$ using an oil bath for overnight (monitored by TLC). Flash column chromatography on silica gel (eluted with Hexane:EtOAc $=95: 5$ ) afforded 13a as a colorless oil (310 $\mathrm{mg}, 0.723 \mathrm{mmol}, 79 \%$ yield).

$R_{\mathrm{f}} 0.50$ (EtOAc:Hex 1:10); $[\alpha]^{23}{ }_{\mathrm{D}}-57.7\left(\mathrm{c} 0.83, \mathrm{CHCl}_{3}\right) ;{ }^{1} \mathrm{H}$ NMR $\left(500 \mathrm{MHz}, \mathrm{CDCl}_{3}\right) \delta$ 7.37-7.27 (m, 5H), $6.02(\mathrm{~d}, J$ $=10.3 \mathrm{~Hz}, 1 \mathrm{H}$ ), 5.73 (ddd, J=10.2, 2.5, $2.0 \mathrm{~Hz}, 1 \mathrm{H}), 5.49$ (d, $J=1.3 \mathrm{~Hz}, 1 \mathrm{H}), 5.27(\mathrm{~d}, J=1.53 \mathrm{~Hz}, 1 \mathrm{H}), 5.06(\mathrm{~s}, 1 \mathrm{H})$, $4.60(\mathrm{~s}, 2 \mathrm{H}), 4.05(\mathrm{~d}, J=9.0,1.3 \mathrm{~Hz}, 1 \mathrm{H}), 3.96-3.93(\mathrm{~m}, 2 \mathrm{H}), 3.64-3.59(\mathrm{~m}, 2 \mathrm{H}), 1.88-1.87(\mathrm{~m}, 2 \mathrm{H}), 1.81(\mathrm{~s}, 3 \mathrm{H})$, 
1.74-1.71 (m, 2H), 1.54-1.51 (m, 1H), 1.43-1.15 (m, 11H); $\left.{ }^{13} \mathrm{C} \mathrm{NMR} \mathrm{(125} \mathrm{MHz,} \mathrm{CDCl}_{3}\right) \delta$ 138.1, 138.0, 130.6, 128.5, 127.9, 127.8, 127.4, 124.7, 93.9, 92.6, 78.6, 76.0, 73.3 , 72.8, 71.5, 65.6, 33.9, 32.2 , 25.7, 24.4, 24.2, 19.5, 19.4, 18.3; IR (NaCl) v 2932, 2857, 1451, 1378, 1295, 1160, 1086, 1049, 826, 733, $698 \mathrm{~cm}^{-1}$; HRMS (ESI) m/z: [M $+\mathrm{Na}]^{+}$Calcd for $\mathrm{C}_{26} \mathrm{H}_{36} \mathrm{NaO}_{5}$ 451.2455; Found 451.2457.

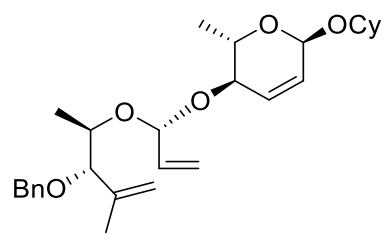

(2S,3R,6R)-3-(((R)-1-(((2R,3S)-3-(benzyloxy)-4-methylpent-4-en-2-yl)oxy)allyl)oxy)-6-(cyclohexyloxy)-2-methyl-3,6-dihydro-2H-pyran (12b): Following the general procedure, to a solution of $\mathrm{Pd}_{2} \mathrm{dba}_{3}(6 \mathrm{mg}, 6.60 \mu \mathrm{mol}$ ) and $(\boldsymbol{R}, \boldsymbol{R})-\mathbf{L}(9.1 \mathrm{mg}, 13.2 \mu \mathrm{mol})$ in toluene, a solution of $\mathbf{5}(92 \mathrm{mg}, 0.377 \mathrm{mmol})$, alcohol 11 (41.0 mg, 0.188 $\mathrm{mmol}$ ), and triethylamine $(2.6 \mu \mathrm{L}, 18.8 \mu \mathrm{mol})$ in toluene (total concentration $0.5 \mathrm{M}$ ) was added. The reaction mixture was heated at $40^{\circ} \mathrm{C}$ using an oil bath for $1 \mathrm{~h}$ (monitored by TLC). The crude was directly purified by flash column chromatography (eluent: hexane/EtOAc 95:5) on silica gel (deactivated by triethylamine) to give acyclic $O, O$-acetal $\mathbf{1 2 b}$ as colorless oil $(85 \mathrm{mg}, 0.186 \mathrm{mmol}, 99 \%)$. The diastereomeric ratio of the crude was calculated as $<1: 25$ by ${ }^{1} \mathrm{H}$ NMR spectroscopy of the crude mixture (The reaction with 1 equiv of allene and 2 equiv of alcohol also showed diastereomeric ratio $<1: 25$ and $96 \%$ yield).

$R_{\mathrm{f}} 0.70$ (EtOAc:Hex 1:10); $[\alpha]^{28}{ }_{\mathrm{D}}-26.3\left(\mathrm{c} 0.55 \mathrm{CHCl}_{3}\right) ;{ }^{1} \mathrm{H}$ NMR $\left(500 \mathrm{MHz}, \mathrm{CDCl}_{3}\right) \delta$ 7.33-7.26 (m, 5H), $6.03(\mathrm{~d}, J=$ $10.2 \mathrm{~Hz}, 1 \mathrm{H}$ ), 5.78 (ddd, $J=17.0,10.3,6.2 \mathrm{~Hz}, 1 \mathrm{H}), 5.64$ (ddd, $J=10.3,4.7,2.3,1 \mathrm{H}), 5.32(\mathrm{~d}, J=17.2 \mathrm{~Hz}, 1 \mathrm{H}$ ), $5.25(\mathrm{~d}, J=10.4 \mathrm{~Hz}, 1 \mathrm{H}), 5.07-5.05(\mathrm{~m}, 3 \mathrm{H}), 4.91(\mathrm{~d}, J=6.3 \mathrm{~Hz}, 1 \mathrm{H}), 4.54(\mathrm{~d}, J=11.8 \mathrm{~Hz}, 1 \mathrm{H}), 4.32(\mathrm{~d}, J=11.8 \mathrm{~Hz}$, 1H), 3.8-3.78 (m, 3H), $3.67(\mathrm{~d}, J=6.1 \mathrm{~Hz}, 1 \mathrm{H}), 3.62-3.57(\mathrm{~m}, 1 \mathrm{H}), 1.90-1.85(\mathrm{~m}, 2 \mathrm{H}), 1.76-1.72(\mathrm{~m}, 5 \mathrm{H}), 1.54-1.51$ (m, 1H), 1.42-1.14 (m, 11H); ${ }^{13} \mathrm{CNMR}\left(125 \mathrm{MHz}, \mathrm{CDCl}_{3}\right) \delta 142.2,138.6,136.2,132.3,128.2,127.7,127.4,126.7$, $118.2,115.8,103.3,92.7,86.0,76.0,75.2$, 71.8, 70.3, 65.8, 33.9, 32.2, 25.7, 24.4, 24.2, 18.3, 18.1, 17.2; IR ( $\mathrm{NaCl})$ v 3031, 2931, 2857, 1452, 1374, 1300, 1152, 1103, 1029, 903, 827, $735 \mathrm{~cm}^{-1}$; HRMS (ESI) m/z: [M+Na] Calcd for $\mathrm{C}_{28} \mathrm{H}_{40} \mathrm{NaO}_{5}$ 479.2768; Found 479.2769.

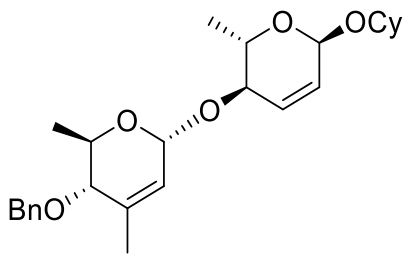

(2R,3S,6R)-3-(benzyloxy)-6-(((2S,3R,6R)-6-(cyclohexyloxy)-2-methyl-3,6-dihydro-2H-pyran-3-yl)oxy)-2,4-dimethyl-3,6-dihydro-2H-pyran (13b): Following the general procedure, $\mathbf{1 2 b}$ (85 $\mathrm{mg}, 0.186 \mathrm{mmol}$ ) was reacted 
with the Hoveyda Grubbs' $2^{\text {nd }}$ catalyst $(5.8 \mathrm{mg}, 9.30 \mu \mathrm{mol})$ in $\mathrm{CH}_{2} \mathrm{Cl}_{2}(2 \mathrm{~mL})$ at $40^{\circ} \mathrm{C}$ using an oil bath for overnight (monitored by TLC). Flash column chromatography on silica gel (eluted with Hexane:EtOAc $=95: 5$ ) afforded $13 \mathrm{~b}$ as a colorless oil (70 $\mathrm{mg}, 0.163 \mathrm{mmol}, 87 \%$ yield).

$R_{\mathrm{f}} 0.5$ (EtOAc:Hex 1:10); $[\alpha]^{23}{ }_{\mathrm{D}}-74.6\left(\mathrm{c} 0.76, \mathrm{CHCl}_{3}\right) ;{ }^{1} \mathrm{H} \mathrm{NMR}\left(500 \mathrm{MHz}, \mathrm{CDCl}_{3}\right) \delta$ 7.36-7.27 (m, 5H), $5.98(\mathrm{~d}, J=$ $10.3 \mathrm{~Hz}, 1 \mathrm{H}), 5.69(\mathrm{ddd}, J=10.2,4.8,2.3,1 \mathrm{H}), 5.52-5.51(\mathrm{~m}, 1 \mathrm{H}), 5.06(\mathrm{~m}, 1 \mathrm{H}), 5.00(\mathrm{~m}, 1 \mathrm{H}), 4.63(\mathrm{~d}, J=11.2$ $\mathrm{Hz}, 1 \mathrm{H}), 4.55(\mathrm{~d}, J=11.2 \mathrm{~Hz}, 1 \mathrm{H}), 4.11-4.05(\mathrm{~m}, 1 \mathrm{H}), 3.98-3.92(\mathrm{~m}, 1 \mathrm{H}), 3.84(\mathrm{dd}, J=9.1,1.4 \mathrm{~Hz}, 1 \mathrm{H}), 3.69(\mathrm{~d}, J$ $=9.0 \mathrm{~Hz}, 1 \mathrm{H}), 3.63-3.58(\mathrm{~m}, 1 \mathrm{H}), 1.89-1.86(\mathrm{~m}, 2 \mathrm{H}), 1.80(\mathrm{~s}, 3 \mathrm{H}), 1.74-1.71(\mathrm{~m}, 2 \mathrm{H}), 1.54-1.51(\mathrm{~m}, 1 \mathrm{H}), 1.43-1.13$ (m, 11H); ${ }^{13} \mathrm{C} \mathrm{NMR}\left(125 \mathrm{MHz}, \mathrm{CDCl}_{3}\right) \delta 140.2,138.2,132.6,128.4,127.7,127.6,127.0,122.5,96.8,92.6,79.6$, 77.6, 75.9, 72.6, 65.6, 65.6, 33.9, 32.2, 25.6, 24.4, 24.2, 18.5, 18.3, 18.0; IR (NaCl) v 2972, 2932, 2857, 1497, 1451,1297, 1192, 1141, 1123, 1078, 734, $698 \mathrm{~cm}^{-1}$; HRMS (ESI) m/z: [M+Na] Calcd for $\mathrm{C}_{26} \mathrm{H}_{36} \mathrm{NaO}_{5}$ 451.2455; Found 451.2458 .

\section{Synthesis of 3-amino-3-methyl disaccharide unit 17 of Saccharomicin}

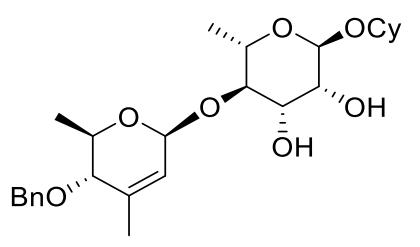

(2R,3R,4S,5R,6S)-5-(((2S,5S,6R)-5-(benzyloxy)-4,6-dimethyl-5,6-dihydro-2H-pyran-2-yl)oxy)-2-(cyclohexyloxy)-6-methyltetrahydro-2H-pyran-3,4-diol (14): To a solution of, 13 a (310 mg, $0.723 \mathrm{mmol}$ ) in acetone/THF (1:1 ratio by volume, $0.72 \mathrm{~mL}$ ), 4-methylmorpholine $\mathrm{N}$-oxide $(110 \mathrm{mg}, 0.940 \mu \mathrm{mol}), \mathrm{OsO}_{4}$ solution $(4 \mathrm{wt} \%$ in $\left.\mathrm{H}_{2} \mathrm{O}, 0.14 \mathrm{~mL}, 21.7 \mu \mathrm{mol}\right)$ and distilled $\mathrm{H}_{2} \mathrm{O}(0.45 \mathrm{~mL}, 1.6 \mathrm{M})$ were added. The reaction mixture was stirred until for $2.5 \mathrm{~h}$ at room temperature. The reaction mixture was diluted with $\mathrm{CH}_{2} \mathrm{Cl}_{2}$ and washed with $10 \% \mathrm{Na}_{2} \mathrm{SO}_{3}$ aqueous solution and sat. $\mathrm{NH}_{4} \mathrm{Cl}$ aqueous solution. The combined organic layers was dried over anhydrous $\mathrm{Na}_{2} \mathrm{SO}_{4}$, and concentrated under reduced pressure. The crude product was isolated by Flash column chromatography on silica gel (deactivated with triethylamine before use, eluted with Hexane:EtOAc $=50: 50$ ) afforded 14 as a colorless oil ( $270 \mathrm{mg}, 0.584 \mathrm{mmol}, 81 \%$ yield).

$R_{\mathrm{f}} 0.40$ (EtOAc:Hex 5:5); $[\alpha]^{23} \mathrm{D}-26.7$ (c 1.04, $\mathrm{CHCl}_{3}$ ); ${ }^{1} \mathrm{H} \mathrm{NMR}\left(500 \mathrm{MHz}, \mathrm{CDCl}_{3}\right) \delta$ 7.37-7.28 (m, 5H), 5.45 (d, $J=$ $1.5 \mathrm{~Hz}, 1 \mathrm{H}), 5.42(\mathrm{~d}, J=1.4 \mathrm{~Hz} 1 \mathrm{H}), 4.93(\mathrm{~s}, 1 \mathrm{H}), 4.67(\mathrm{~d}, J=10.9 \mathrm{~Hz}, 1 \mathrm{H}), 4.62(\mathrm{~d}, J=0.7 \mathrm{~Hz}, 1 \mathrm{H}), 4.60(\mathrm{~d}, J=10.9$ $\mathrm{Hz}, 1 \mathrm{H}), 3.92-3.91(\mathrm{~m}, 1 \mathrm{H}), 3.82-3.76(\mathrm{~m}, 3 \mathrm{H}), 3.68(\mathrm{~d}, J=8.2 \mathrm{~Hz}, 1 \mathrm{H}), 3.61-3.56(\mathrm{~m}, 2 \mathrm{H}), 2.72(\mathrm{~d}, J=1.7 \mathrm{~Hz}, 1 \mathrm{H})$, 1.84-1.79 (m, 5H), 1.72-1.69 (m, 2H), 1.52-1.49 (m, 1H), 1.43-1.16 (m, 11H); ${ }^{13} \mathrm{C} \mathrm{NMR}\left(125 \mathrm{MHz}, \mathrm{CDCl}_{3}\right) \delta 142.3$, 137.7, 128.5, 128.0, 127.9,123.2, 96.7, 95.8, 79.0, 76.7, 74.7, 74.5, 73.1, 71.2, 70.1, 66.0, 33.3, 31.3, 25.6, 23.8, $18.9,18.6,18.0$; IR (NaCl) v 3426, 3032, 292, 2857, 1674, 1451, 1383, 1062, 984, 894, 839, $735 \mathrm{~cm}^{-1}$; HRMS (ESI) $\mathrm{m} / \mathrm{z}:[\mathrm{M}+\mathrm{Na}]^{+}$Calcd for $\mathrm{C}_{26} \mathrm{H}_{38} \mathrm{NaO}_{7}[\mathrm{M}+\mathrm{Na}]^{+}$485.2510; Found 485.2512. 


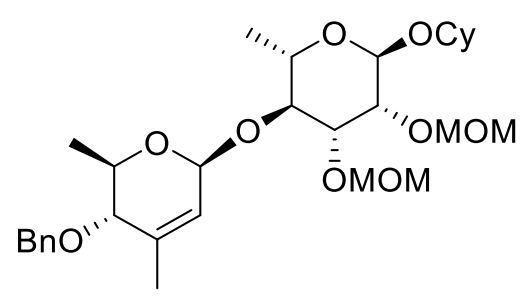

(2R,3S,6S)-3-(benzyloxy)-6-(((2S,3S,4R,5R,6R)-6-(cyclohexyloxy)-4,5-bis(methoxymethoxy)-2-methyltetrahydro-2H-pyran-3-yl)oxy)-2,4-dimethyl-3,6-dihydro-2H-pyran (15): To a solution of 14 (30 mg, 0.0648 mmol) in $\mathrm{CH}_{2} \mathrm{Cl}_{2}(0.3 \mathrm{~mL}), \mathrm{N}, \mathrm{N}$-diisopropylethylamine $(0.06 \mathrm{~mL}, 0.324 \mathrm{mmol})$ and methoxymethylchloride $(15.0 \mu \mathrm{L}$, $0.194 \mathrm{mmol}$ ) were added. The reaction mixture was heated at $40^{\circ} \mathrm{C}$ using an oil bath for overnight (monitored by TLC) and quenched with sat. $\mathrm{NH}_{4} \mathrm{Cl}$ solution. The crude product was extracted with $\mathrm{CH}_{2} \mathrm{Cl}_{2}(3 \times 5 \mathrm{~mL})$, dried over $\mathrm{Na}_{2} \mathrm{SO}_{4}$ and concentrated under reduced pressure. The column chromatography of crude on silica gel (eluted with EtOAc:Hex = 3:10) afforded 15 as colorless oil (32 mg, $0.0581 \mathrm{mmol}, 89 \%$ ).

$R_{\mathrm{f}} 0.8$ (EtOAc:Hex 3:10); $[\alpha]^{23} \mathrm{D}-74.7\left(c \mathrm{c} 1.00, \mathrm{CHCl}_{3}\right) ;{ }^{1} \mathrm{H} \mathrm{NMR}\left(500 \mathrm{MHz}, \mathrm{CDCl}_{3}\right) \delta$ 7.36-7.26 (m, 5H), $5.52(\mathrm{~s}, 1 \mathrm{H})$, $5.32(\mathrm{~s}, 1 \mathrm{H}), 4.94(\mathrm{~s}, 1 \mathrm{H}), 4.79-4.74(\mathrm{~m}, 4 \mathrm{H}), 4.63(\mathrm{~d}, J=11.0 \mathrm{~Hz}, 1 \mathrm{H}), 4.58(\mathrm{~d}, J=11.0 \mathrm{~Hz}, 1 \mathrm{H}), 3.96-3.95(\mathrm{~m}, 1$ H), $3.85(\mathrm{~m}, 1 \mathrm{H}), 3.76-3.66(\mathrm{~m}, 4 \mathrm{H}), 3.54(\mathrm{~m}, 1 \mathrm{H}), 3.40(\mathrm{~s}, 6 \mathrm{H}), 1.83-1.69(\mathrm{~m}, 7 \mathrm{H}), 1.51-1.49(\mathrm{~m}, 1 \mathrm{H}), 1.35-1.17$ (m, 11H); ${ }^{13} \mathrm{C} \mathrm{NMR}\left(125 \mathrm{MHz}, \mathrm{CDCl}_{3}\right) \delta$ 139.0, 1381, 128.4, 127.9, 127.8, 125.1, 98.4, 97.0, 96.6, 96.5, 79.6, 78.6, $77.4,76.6,75.1$, 73.4, 71.5, 67.5, 55.8, 55.5, 33.3, 31.5, 25.6, 24.1, 23.9, 19.0, 18.9, 18.0 ; IR (NaCl) v 2934, 2858, $1451,1384,1152,1114,1052,1028,919,842,736,699 \mathrm{~cm}^{-1}$; HRMS (ESI) m/z: [M+Na] ${ }^{+}$Calcd for $\mathrm{C}_{30} \mathrm{H}_{46} \mathrm{NaO}_{9}$ 573.3034; Found 573.3036.

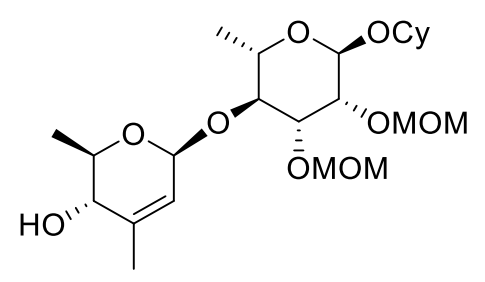

(2R,3S,6S)-6-(((2S,3S,4R,5R,6R)-6-(cyclohexyloxy)-4,5-bis(methoxymethoxy)-2-methyltetrahydro-2H-pyran3-yl)oxy)-2,4-dimethyl-3,6-dihydro-2H-pyran-3-ol (S5): In a two-neck flame-dried round bottom flask, fitted with Dewar flask (cooled to $-78{ }^{\circ} \mathrm{C}$ ), $\mathrm{NH}_{3}$ gas $\left(5 \mathrm{~mL}\right.$ ) was trapped at $-78{ }^{\circ} \mathrm{C}$. To this mixture, Na metal $(13 \mathrm{mg}$, $0.290 \mathrm{mmol}$ ) was added, followed by the addition of solution of 15 (32 mg, $0.0581 \mathrm{mmol})$ in THF (2.0 mL). The reaction mixture was stirred at $-78^{\circ} \mathrm{C}$ for $2 \mathrm{~h}$ (monitored by TLC) and allowed to warm to room temperature. The reaction mixture was quenched with solid $\mathrm{NH}_{4} \mathrm{Cl}$ solution until blue color disappeared, and was extracted with EtOAc $(3 \times 10 \mathrm{~mL})$. The combined organic layers was dried over $\mathrm{Na}_{2} \mathrm{SO}_{4}$ and concentrated under reduced pressure to give crude. The Flash column chromatography of crude on silica gel (eluted with EtOAc:Hex = 5:5) afforded S5 (25 mg, $0.0543 \mathrm{mmol}, 93 \%)$. 
$R_{\mathrm{f}} 0.4$ (EtOAc:Hex 5:5); $[\alpha]^{31} \mathrm{D}-100.0$ (c 1.05, $\mathrm{CHCl}_{3}$ ); ${ }^{1} \mathrm{H}$ NMR $\left(500 \mathrm{MHz}, \mathrm{CDCl}_{3}\right) \delta 5.49$ (d, J=1.2 Hz, $1 \mathrm{H}$ ), 5.30 (d, $J=1.3 \mathrm{~Hz}, 1 \mathrm{H}), 4.93(\mathrm{~d}, J=1.6 \mathrm{~Hz}, 1 \mathrm{H}), 4.78-4.73(\mathrm{~m}, 4 \mathrm{H}), 3.97-3.92(\mathrm{~m}, 1 \mathrm{H}), 3.85-3.84(\mathrm{~m}, 1 \mathrm{H}), 3.75-3.69(\mathrm{~m}$, $3 \mathrm{H}), 3.54-3.46(\mathrm{~m}, 2 \mathrm{H}), 3.41(\mathrm{~s}, 3 \mathrm{H}), 3.40(\mathrm{~s}, 3 \mathrm{H}), 1.84-1.78(\mathrm{~m}, 5 \mathrm{H}), 1.69-1.68(\mathrm{~m}, 2 \mathrm{H}), 1.63(\mathrm{~m}, 1 \mathrm{H}), 1.51-1.48$ (m, 1H), 1.36-1.15 (m, 11H); $\left.{ }^{13} \mathrm{C} \mathrm{NMR} \mathrm{(125} \mathrm{MHz,} \mathrm{CDCl}\right)$ $\delta$ 139.5, 124.5, 98.3, 97.0, 96.5, 96.4, 78.6, 77.4, 76.6, $75.1,73.5,72.0,67.5,55.8,55.6,33.3,31.5,25.6,24.2,23.9,18.4,18.0 ; \mathrm{IR}(\mathrm{NaCl}) \vee 3471,2934,2858,1450$, 1384, 1215, 1190, 1153, 1051, 1028, 952, $919 \mathrm{~cm}^{-1}$; HRMS (ESI) m/z: [M+Na] ${ }^{+}$Calcd for $\mathrm{C}_{23} \mathrm{H}_{40} \mathrm{NaO}_{9}$ 483.2565; Found 483.2567.

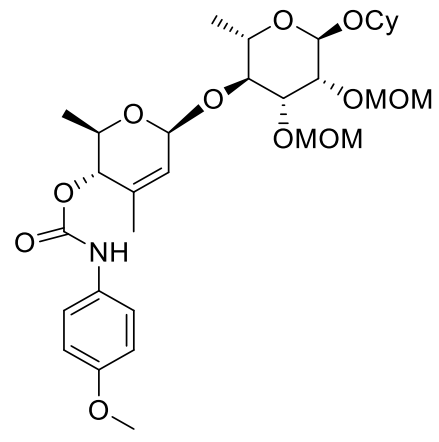

(2R,3S,6S)-6-(((2S,3S,4R,5R,6R)-6-(cyclohexyloxy)-4,5-bis(methoxymethoxy)-2-methyltetrahydro-2H-pyran3-yl)oxy)-2,4-dimethyl-3,6-dihydro-2H-pyran-3-yl (4-methoxyphenyl)carbamate (16): To a solution of S5 (83 $\mathrm{mg}, 0.1802 \mathrm{mmol})$ in $\mathrm{CH}_{2} \mathrm{Cl}_{2}(1.8 \mathrm{~mL})$, p-methoxyphenyl isocyanate $(25 \mu \mathrm{L}, 0.198 \mathrm{mmol})$ and 1,8-diazabicyclo[5.4.0]undec-7-ene, DBU $(2.70 \mu \mathrm{L}, 18.02 \mu \mathrm{mol})$ were added at room temperature. After stirring reaction mixture for $2.5 \mathrm{~h}$ (monitored by TLC), the mixture was concentrated under reduced pressure. The column chromatography of crude on silica gel (eluted with EtOAc:Hex = 3:10) afforded 16 as white solid (91 mg, 0.149 $\mathrm{mmol}, 83 \%)$.

m.p. $52-54{ }^{\circ} \mathrm{C} ; R_{\mathrm{f}} 0.5$ (EtOAc:Hex 3:10); $[\alpha]^{30}{ }_{\mathrm{D}}-50.7$ (c 0.51, $\left.\mathrm{CHCl}_{3}\right) ;{ }^{1} \mathrm{H}$ NMR $\left(500 \mathrm{MHz}, \mathrm{CDCl}_{3}\right) \delta 7.30-7.29(\mathrm{~m}$, 2H), 6.86-6.84 (m, 2H), $6.54(\mathrm{bs}, 1 \mathrm{H}), 5.60(\mathrm{~s}, 1 \mathrm{H}), 5.35(\mathrm{~d}, J=0.9 \mathrm{~Hz}, 1 \mathrm{H}), 5.13(\mathrm{~d}, J=6.9 \mathrm{~Hz}, 1 \mathrm{H}), 4.95(\mathrm{~d}, J=1.6$ $\mathrm{Hz}, 1 \mathrm{H}), 4.79-4.74(\mathrm{~m}, 4 \mathrm{H}), 3.97-3.93(\mathrm{~m}, 1 \mathrm{H}), 3.86-3.85(\mathrm{~m}, 1 \mathrm{H}), 3.78(\mathrm{~s}, 3 \mathrm{H}), 3.76-3.68(\mathrm{~m}, 3 \mathrm{H}), 3.56-3.51(\mathrm{~m}$, $1 \mathrm{H}), 3.42(\mathrm{~s}, 3 \mathrm{H}), 3.41(\mathrm{~s}, 3 \mathrm{H}), 1.85-1.81(\mathrm{~m}, 2 \mathrm{H}), 1.74-1.69(\mathrm{~m}, 5 \mathrm{H}), 1.52-1.50(\mathrm{~m}, 1 \mathrm{H}), 1.38-1.17, \mathrm{~m}, 11 \mathrm{H}) ;{ }^{13} \mathrm{C}$ NMR $\left(125 \mathrm{MHz}, \mathrm{CDCl}_{3}\right) \delta 156.2,153.6,136.6,130.7,125.9,120.6,114.3,98.0,97.0,96.6,96.5,78.7,77.5,76.6$, 75.1, 73.2, 71.1, 67.5, 55.8, 55.5, 33.0, 31.5, 25.6, 24.1, 23.9, 18.5, 18.4, 18.0 ; IR (NaCl) v 3327, 2939, 2857, 1733, 1601, 1517, 1449, 1385, 1297, 1217, 1051, $1030 \mathrm{~cm}^{-1}$; HRMS (ESI) m/z: [M+Na] ${ }^{+}$Calcd for $\mathrm{C}_{31} \mathrm{H}_{47} \mathrm{NNaO}_{11}$ 632.3041; Found 632.3044. 


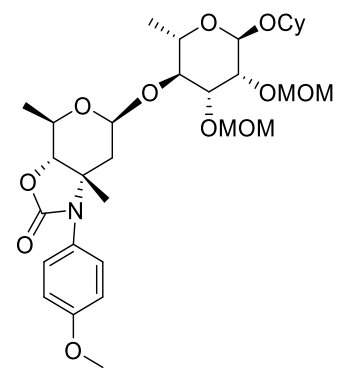

(3aS,4R,6S,7aS)-6-(((2S,3S,4R,5R,6R)-6-(cyclohexyloxy)-4,5-bis(methoxymethoxy)-2-methyltetrahydro-2Hpyran-3-yl)oxy)-1-(4-methoxyphenyl)-4,7a-dimethylhexahydro-2H-pyrano[4,3-d]oxazol-2-one (17): To a solution of 16 (25 mg, $0.041 \mathrm{mmol})$ in THF:DMSO (10:1) (2 mL) in a seal-tube, sodium bicarbonate (41 mg, 0.492 $\mathrm{mmol}$ ) and 2-iodoxybenzoic acid (69 $\mathrm{mg}, 0.246 \mathrm{mmol}$ ) were added, and reaction mixture was heated at $90{ }^{\circ} \mathrm{C}$ using an oil bath for $12 \mathrm{~h}$ (monitored by TLC). The mixture was allowed to cool to room temperature and was diluted with EtOAc $(5 \mathrm{~mL})$. The organic layer was washed with $5 \% \mathrm{NaHCO}_{3}(3 \times 5 \mathrm{~mL})$, dried over $\mathrm{Na}_{2} \mathrm{SO}_{4}$, and concentrated under reduced pressure. The column chromatography of crude on silica gel (eluted with EtOAc:Hex = 3:10) afforded 17 as white solid $(18.7 \mathrm{mg}, 0.0307 \mathrm{mmol}, 75 \%)$.

m.p. 51-53 ${ }^{\circ} \mathrm{C} ; R_{\mathrm{f}} 0.3$ (EtOAc:Hex 3:10); $[\alpha]^{31} \mathrm{D}-52.4$ (c 0.57, $\left.\mathrm{CHCl}_{3}\right) ;{ }^{1} \mathrm{H}$ NMR $\left(500 \mathrm{MHz}, \mathrm{CDCl}_{3}\right) \delta$ 7.15-7.13 (m, 2H), 6.94-6.92 (m, 2H), $4.99(\mathrm{dd}, J=9.2,2.2 \mathrm{~Hz}, 1 \mathrm{H}), 4.94(\mathrm{~d}, J=1.5 \mathrm{~Hz}, 1 \mathrm{H}), 4.79(\mathrm{~d}, J=6.9 \mathrm{~Hz}, 1 \mathrm{H}), 4.76(\mathrm{~d}, J$ = $6.9 \mathrm{~Hz}, 1 \mathrm{H}), 4.64(\mathrm{~s}, 2 \mathrm{H}), 3.90-3.82(\mathrm{~m}, 6 \mathrm{H}), 3.73-3.63(\mathrm{~m}, 3 \mathrm{H}), 3.56-3.51(\mathrm{~m}, 1 \mathrm{H}), 3.42(\mathrm{~s}, 3 \mathrm{H}), 3.33(\mathrm{~s}, 3 \mathrm{H})$, $2.05(\mathrm{dd}, J=14.85,2.16 \mathrm{~Hz}, 1 \mathrm{H}), 1.89-1.81(\mathrm{~m}, 2 \mathrm{H}), 1.72-1.66(\mathrm{~m}, 2 \mathrm{H}), 1.55-1.50(\mathrm{~m}, 2 \mathrm{H}), 1.42-1.15, \mathrm{~m}, 14 \mathrm{H})$; ${ }^{13} \mathrm{C}$ NMR $\left(125 \mathrm{MHz}, \mathrm{CDCl}_{3}\right) \delta$ 159.4, 156.8, 130.3, 126.9, 114.7, 99.3, 97.1, 96.6, 96.1, 80.9, 78.6, 77.1, 76.5, 75.3, 70.5, 67.4, 62.6, 55.7, 55.6, 55.5, 37.4, 33.4, 31.6, 26.7, 25.6, 24.2, 23.9, 18.6, 17.9; IR (NaCl) v 2934, 2857, 1763, 1514, 1451, 1373, 1296, 1251, 1160, 1031, 919, $834 \mathrm{~cm}^{-1}$; HRMS (ESI) m/z: [M+Na] Calcd for $\mathrm{C}_{31} \mathrm{H}_{47} \mathrm{NNaO}_{11}$ 632.3041; Found 632.3043.

\section{Synthesis of Fucose-Rhamnose disaccharide 23 by protective group-less coupling}

1) synthesis of $\mathbf{2 1 a}$

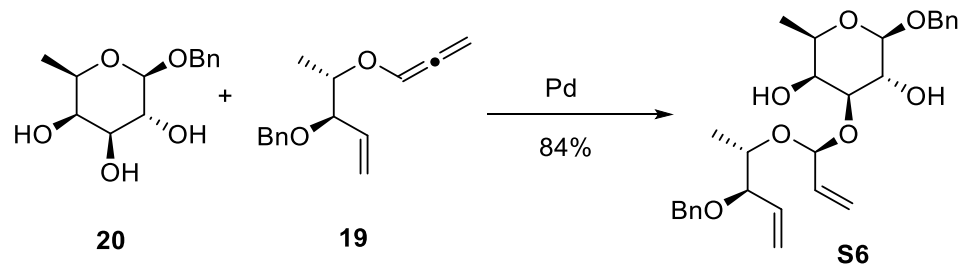

S6

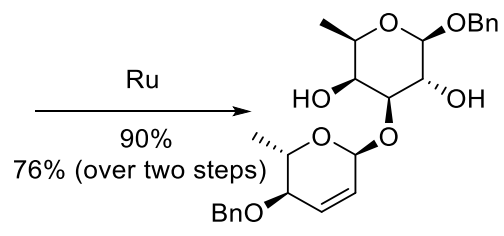

$21 \mathrm{a}$<smiles>C=C[C@H](O[C@H](C)OCc1ccccc1)[C@@H](C)O[C@H]1[C@@H](O)[C@H](Cc2ccccc2)O[C@H](C)[C@H]1O</smiles> 
(2R,3R,4S,5S,6R)-2-(benzyloxy)-4-(((S)-1-(((2S,3R)-3-(benzyloxy)pent-4-en-2-yl)oxy)allyl)oxy)-6-methyltetrahydro-2H-pyran-3,5-diol (S6): To a solution of $\mathrm{Pd}_{2} \mathrm{dba}_{3}(2.7 \mathrm{mg}, 2.95 \mu \mathrm{mol}),(\mathbf{S}, \mathbf{S})-\mathbf{3}$ (4.1 mg, $\left.5.90 \mu \mathrm{mol}\right)$ and $20^{4}$ (30.1 mg, $0.118 \mathrm{mmol})$ in dichloromethane, a solution of $19^{3}(30.2 \mathrm{mg}, 0.129 \mathrm{mmol})$ and triethylamine $(1.7 \mu \mathrm{L}$, $11.80 \mu \mathrm{mol}$ ) in dichloromethane (total concentration $0.5 \mathrm{M}$ ) was added. The reaction mixture was stirred at $0{ }^{\circ} \mathrm{C}$ for $20 \mathrm{~h}$ (monitored by TLC). The crude was directly purified by flash column chromatography (eluent: hexane/EtOAc 90:10) on silica gel to afford acyclic 0,0 -acetal $\mathbf{S 6}$ as colorless oil (48.1 $\mathrm{mg}, 0.099 \mathrm{mmol}, 84 \%$ ) along with minor regioisomers $(8.5 \mathrm{mg}, 0.0175 \mathrm{mmol}, 15 \%)$.

$R_{\mathrm{f}} 0.3$ (EtOAc:Hex 3:10); $[\alpha]^{24}{ }_{\mathrm{D}}-23.4\left(\mathrm{c} 0.65, \mathrm{CH}_{2} \mathrm{Cl}_{2}\right) ;{ }^{1} \mathrm{H}$ NMR 7.38-7.24 (m, 10H), 5.91-5.83 (m, 2H), 5.43-5.38 $(\mathrm{m}, 2 \mathrm{H}), 5.29-5.22(\mathrm{~m}, 3 \mathrm{H}), 4.90(\mathrm{~d}, J=12.0 \mathrm{~Hz}, 1 \mathrm{H}), 4.58(\mathrm{~d}, J=11.9 \mathrm{~Hz}, 1 \mathrm{H}), 4.58(\mathrm{~d}, J=12.0 \mathrm{~Hz}, 1 \mathrm{H}) 4.38(\mathrm{~d}, J$ $=11.9 \mathrm{~Hz}, 1 \mathrm{H}), 4.13-4.08(\mathrm{~m}, 1 \mathrm{H}), 3.96-3.92(\mathrm{~m}, 1 \mathrm{H}), 3.77-3.69(\mathrm{~m}, 4 \mathrm{H}), 3.33-3.29(\mathrm{~m}, 1 \mathrm{H}), 2.78(\mathrm{bs}, 1 \mathrm{H}), 2.50$ (bs, $1 \mathrm{H}), 1.29$ (d, $J=6.5 \mathrm{~Hz}, 3 \mathrm{H}), 1.08$ (d, $J=6.5 \mathrm{~Hz}, 3 \mathrm{H}) ;{ }^{13} \mathrm{C}$ NMR $(125 \mathrm{~Hz})$ 138.3, 137.8, 136.0, 134.6, 128.5, 128.5, 128.1, 128.1, 127.8, 127.8, 120.4, 118.7, 102.9, 102.0, 83.9, 78.3, 75.0, 71.2, 70.8, 70.5, 70.3, 17.1, 16.5; IR (NaCl) v 3476, 2980, 2872, 1373, 1171, 1069, 1028, 737, 698, $511 \mathrm{~cm}^{-1} ;$ HRMS (ESI) m/z: [M+Na] ${ }^{+}$Calcd for $\mathrm{C}_{28} \mathrm{H}_{36} \mathrm{NaO}_{7}$ 507.2353; Found 507.2355.

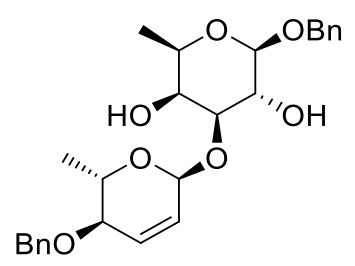

(2R,3R,4S,5S,6R)-2-(benzyloxy)-4-(((2S,5R,6S)-5-(benzyloxy)-6-methyl-5,6-dihydro-2H-pyran-2-yl)oxy)-6-

methyltetrahydro-2H-pyran-3,5-diol (21a): Following the general procedure, $\mathbf{S 6}$ (35 mg, $0.072 \mathrm{mmol}$ ) was reacted with the Hoveyda Grubbs' $2^{\text {nd }}$ catalyst $(4.5 \mathrm{mg}, 7.22 \mu \mathrm{mol})$ in $\mathrm{CH}_{2} \mathrm{Cl}_{2}(0.7 \mathrm{~mL})$ at $40^{\circ} \mathrm{C}$ using an oil bath for overnight (monitored by TLC). Flash column chromatography on silica gel (eluted with Hexane:EtOAc $=10: 3$ ) afforded 21a as a colorless oil ( $29.7 \mathrm{mg}, 0.065 \mathrm{mmol}, 90 \%$ yield, $76 \%$ two-step yield of pure $\mathbf{2 1 a}$ from triol 20). $R_{\mathrm{f}} 0.2$ (EtOAc:Hex 3:10); $[\alpha]^{24} \mathrm{D}-87.6\left(c 0.75, \mathrm{CH}_{2} \mathrm{Cl}_{2}\right) ;{ }^{1} \mathrm{H}$ NMR 7.38-7.28 (m, $\left.10 \mathrm{H}\right), 6.08(\mathrm{~d}, J=10.3 \mathrm{~Hz}, 1 \mathrm{H}), 5.82$ (d, $J=10.3,4.6,2.4 \mathrm{~Hz} 1 \mathrm{H}), 5.27(\mathrm{~m}, 1 \mathrm{H}), 4.94(\mathrm{~d}, J=11.8 \mathrm{~Hz}, 1 \mathrm{H}), 4.67(\mathrm{~d}, J=11.7 \mathrm{~Hz}, 1 \mathrm{H}), 4.60(\mathrm{~d}, J=11.8 \mathrm{~Hz}$, $1 \mathrm{H}), 4.54(\mathrm{~d}, J=11.7 \mathrm{~Hz}, 1 \mathrm{H}), 4.30(\mathrm{~d}, J=7.8 \mathrm{~Hz}, 1 \mathrm{H}), 3.96-3.90(\mathrm{~m}, 1 \mathrm{H}), 3.80-3.75(\mathrm{~m}, 2 \mathrm{H}), 3.70-3.60(\mathrm{~m}, 3 \mathrm{H})$, $2.42(\mathrm{~d}, J=2.2 \mathrm{~Hz}, 1 \mathrm{H}), 2.41(\mathrm{~d}, J=1.9 \mathrm{~Hz}, 1 \mathrm{H}), 1.39$ (d, J = 6.5 Hz, 3H), 1.29 (d, J=6.2 Hz, 3H); ${ }^{13} \mathrm{C} \mathrm{NMR}(125 \mathrm{~Hz})$ 138.1, 137.4, 131.0, 128.6, 128.3, 128.0, 128.0, 126.5, 101.9, 96.2, 80.5, 76.2, 72.1, 71.2, 71.1, 70.8, 70.6, 66.4, 18.5, 16.5; IR (NaCl) v 3500, 2979, 2933, 1454, 1379, 1308, 1070, 1039, 988, 738, $696 \mathrm{~cm}^{-1}$; HRMS (ESI) m/z: $[\mathrm{M}+\mathrm{Na}]^{+}$Calcd for $\mathrm{C}_{26} \mathrm{H}_{32} \mathrm{NaO}_{7}$ 479.2040; Found 479.2041. 


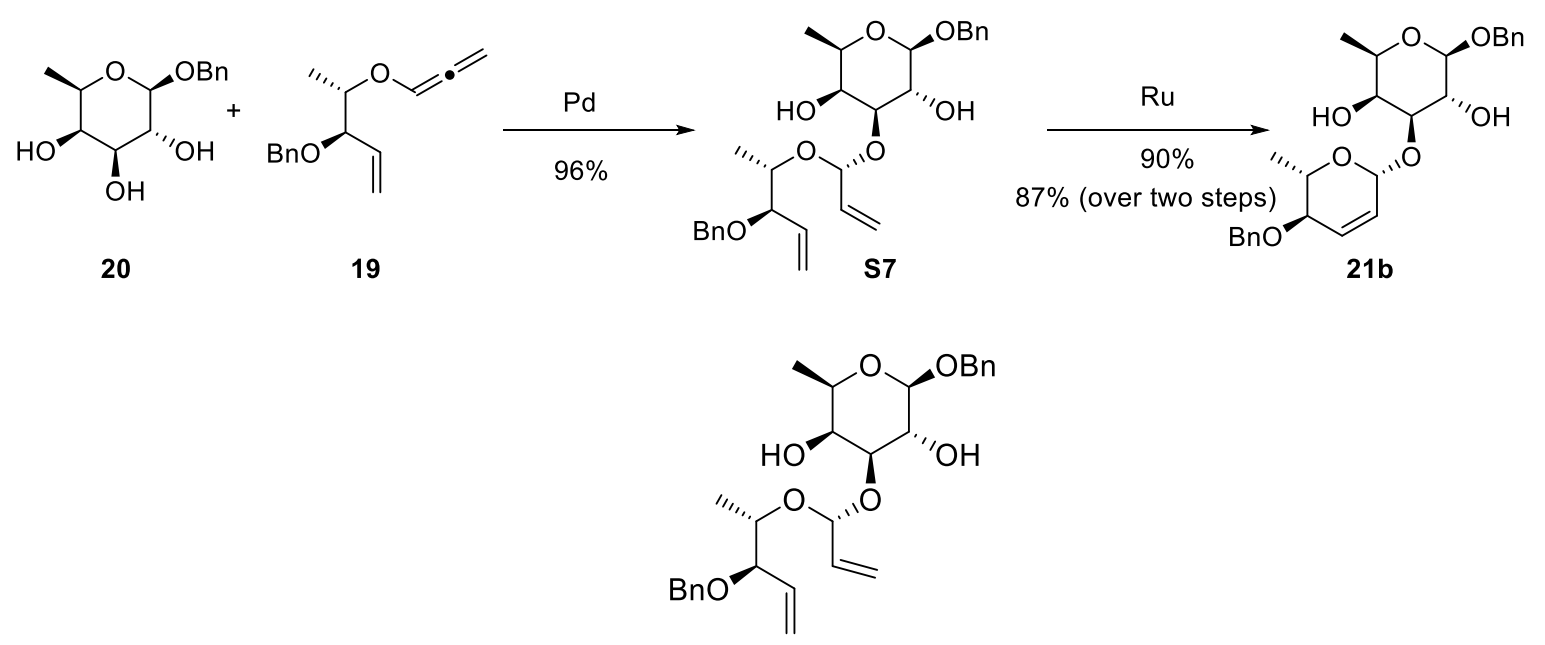

(2R,3R,4S,5S,6R)-2-(benzyloxy)-4-(((R)-1-(((2S,3R)-3-(benzyloxy)pent-4-en-2-yl)oxy)allyl)oxy)-6-methyltetrahydro-2H-pyran-3,5-diol (S7): To a solution of $\mathrm{Pd}_{2} \mathrm{dba}_{3}(2.7 \mathrm{mg}, 2.95 \mu \mathrm{mol}),(\boldsymbol{R}, \boldsymbol{R})-\mathrm{L}(4.1 \mathrm{mg}, 5.90 \mu \mathrm{mol})$ and $2 \mathbf{2 0}^{4}$ (30.1 mg, $0.118 \mathrm{mmol})$ in dichloromethane, a solution of $19^{3}(30.2 \mathrm{mg}, 0.129 \mathrm{mmol})$ and triethylamine $(1.8 \mu \mathrm{L}$, $11.80 \mu \mathrm{mol}$ ) in dichloromethane (total concentration $0.5 \mathrm{M}$ ) was added. The reaction mixture was stirred at $0{ }^{\circ} \mathrm{C}$ for $20 \mathrm{~h}$ (monitored by TLC). The crude was directly purified by flash column chromatography (eluent: hexane/EtOAc 90:10) on silica gel to afford acyclic O,O-acetal $\mathbf{S 7}$ as colorless oil ( $55 \mathrm{mg}, 0.113 \mathrm{mmol}, 96 \%$ ).

$R_{\mathrm{f}} 0.3$ (EtOAc:Hex 3:10); $[\alpha]^{24}{ }_{\mathrm{D}}-40.3\left(\mathrm{c} 0.75, \mathrm{CH}_{2} \mathrm{Cl}_{2}\right) ;{ }^{1} \mathrm{H}$ NMR 7.40-7.25 (m, 10H), 5.88 (ddd, $J=17.1,10.4,5.7$ $\mathrm{Hz}, 1 \mathrm{H}$ ), 5.80 (ddd, $J=18.0,10.3,7.9 \mathrm{~Hz}, 1 \mathrm{H}$ ), 5.40 (d, $J=17.3 \mathrm{~Hz}, 1 \mathrm{H}$ ), 5.36 (d, $J=10.4,1.1 \mathrm{~Hz}, 1 \mathrm{H}$ ), $5.32-5.26$ $(\mathrm{m}, 3 \mathrm{H}), 4.94(\mathrm{~d}, J=11.9 \mathrm{~Hz}, 1 \mathrm{H}), 4.63(\mathrm{~d}, J=11.4 \mathrm{~Hz}, 1 \mathrm{H}), 4.61(\mathrm{~d}, J=11.4 \mathrm{~Hz}, 1 \mathrm{H}) 4.37(\mathrm{~d}, J=11.9 \mathrm{~Hz}, 1 \mathrm{H}), 4.25$ (d, $J=7.8 \mathrm{~Hz}, 1 \mathrm{H}), 4.11-4.07(\mathrm{~m}, 1 \mathrm{H}), 3.77-3.73(\mathrm{~m}, 2 \mathrm{H}), 3.67-3.66(\mathrm{~m}, 1 \mathrm{H}), 3.56(\mathrm{dd}, J=9.5,3.4 \mathrm{~Hz}, 1 \mathrm{H}), 3.50-$ $3.46(\mathrm{~m}, 1 \mathrm{H}), 2.89$ (bs, 1H), 2.38 (bs, 1H), 1.35 (d, $J=6.5 \mathrm{~Hz}, 3 \mathrm{H}), 1.21$ (d, J = 6.5 Hz, 3H); ${ }^{13} \mathrm{C} \mathrm{NMR}(125 \mathrm{~Hz}) 138.5$, 137.6, 135.7, 134.9, 128.5, 128.5, 128.3, 128.0, 127.9, 127.7, 119.9, 118.9, 102.1, 102.0, 83.8, 78.8, 74.5, 70.6, 70.4, 17.4, 16.5; IR (NaCl) v 3470, 2934, 2872, 1497, 1424, 1172, 1069, 994, 737, $698 \mathrm{~cm}^{-1}$; HRMS (ESI) m/z: $[\mathrm{M}+\mathrm{Na}]^{+}$Calcd for $\mathrm{C}_{28} \mathrm{H}_{36} \mathrm{NaO}_{7}$ 507.2353; Found 507.2356.

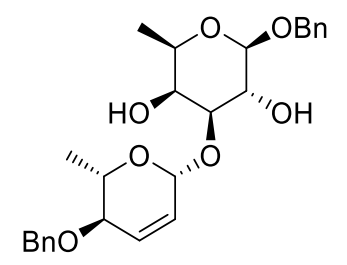

(2R,3R,4S,5S,6R)-2-(benzyloxy)-4-(((2R,5R,6S)-5-(benzyloxy)-6-methyl-5,6-dihydro-2H-pyran-2-yl)oxy)-6methyltetrahydro-2H-pyran-3,5-diol (21b): Following the general procedure, $\mathbf{S 7}$ (40 mg, $0.082 \mathrm{mmol}$ ) was reacted with the Hoveyda Grubbs' $2^{\text {nd }}$ catalyst $(5.2 \mathrm{mg}, 8.25 \mu \mathrm{mol})$ in $\mathrm{CH}_{2} \mathrm{Cl}_{2}(0.8 \mathrm{~mL})$ at $40^{\circ} \mathrm{C}$ using an oil bath for overnight (monitored by TLC). Flash column chromatography on silica gel (eluted with Hexane:EtOAc $=10: 3$ )

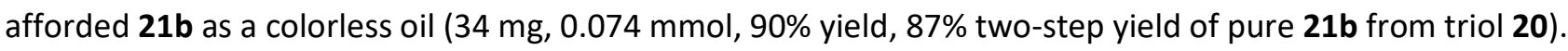


$R_{\mathrm{f}} 0.2$ (EtOAc:Hex 3:10); $[\alpha]^{24} \mathrm{D}-89.5\left(c \mathrm{c} .91, \mathrm{CH}_{2} \mathrm{Cl}_{2}\right.$ ); ${ }^{1} \mathrm{H}$ NMR 7.41-7.27 (m, 10H), $6.10(\mathrm{~d}, J=10.4 \mathrm{~Hz}, 1 \mathrm{H}), 5.82$ (d, J=10.4 Hz, 1H), $5.27(\mathrm{~m}, 1 \mathrm{H}), 4.95(\mathrm{~d}, J=11.9 \mathrm{~Hz}, 1 \mathrm{H}), 4.69(\mathrm{~d}, J=11.9 \mathrm{~Hz}, 1 \mathrm{H}), 4.65(\mathrm{~d}, J=11.6 \mathrm{~Hz}, 1 \mathrm{H})$, $4.55(\mathrm{~d}, J=11.5 \mathrm{~Hz}, 1 \mathrm{H}), 4.36(\mathrm{~d}, J=7.7 \mathrm{~Hz}, 1 \mathrm{H}), 3.80-3.74(\mathrm{~m}, 5 \mathrm{H}), 3.60(\mathrm{dd}, J=9.2,3.3 \mathrm{~Hz}, 1 \mathrm{H}), 3.59-3.50(\mathrm{~m}$, 1H), 2.36 (bs, $1 \mathrm{H}), 1.38$ (d, $J=6.5 \mathrm{~Hz}, 3 \mathrm{H}), 1.35$ (d, $J=5.8 \mathrm{~Hz}, 3 \mathrm{H}) ;{ }^{13} \mathrm{C}$ NMR (125 Hz) 137.9, 137.6, 131.0, 128.7, 128.5, 128.3, 128.2, 128.1, 127.8, 101.8, 97.1, 83.1, 75.4, 73.4, 71.8, 71.1, 70.6, 70.4, 69.8, 18.7, 16.4; IR (NaCl) v 3452, 2979, 2872, 1497, 1454, 1303, 1164, 1071, 738, $698 \mathrm{~cm}^{-1}$; HRMS (ESI) m/z: [M+Na] ${ }^{+}$Calcd for $\mathrm{C}_{26} \mathrm{H}_{32} \mathrm{NaO}_{7}$ 479.2040; Found 479.2042.

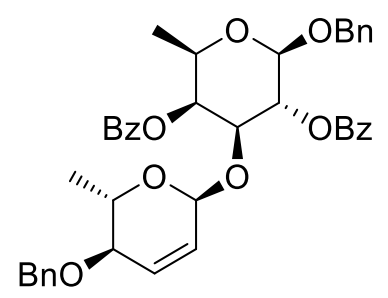

\section{(2R,3R,4S,5S,6R)-2-(benzyloxy)-4-(((2S,5R,6S)-5-(benzyloxy)-6-methyl-5,6-dihydro-2H-pyran-2-yl)oxy)-6-}

methyltetrahydro-2H-pyran-3,5-diyl dibenzoate (22): To a solution of 21a (30 mg, $0.066 \mathrm{mmol}$ ), in pyridine $(0.35 \mathrm{~mL})$, was added benzoyl chloride $(17 \mu \mathrm{L}, 0.144 \mathrm{mmol})$ at $0{ }^{\circ} \mathrm{C}$. The reaction mixture was stirred at room temperature for $12 \mathrm{~h}$ (monitored by TLC). The reaction was quenched with water and extracted with ethyl acetate $(5 \mathrm{~mL})$. The organic layer was washed with water $(3 \times 5 \mathrm{~mL})$, dried over sodium sulfate, concentrated under reduced pressure to obtain crude. The crude was purified by column chromatography (eluent: hexane/EtOAc 70:30) on silica gel to afford 22 as white solid (36.7 $\mathrm{mg}, 0.057 \mathrm{mmol}, 86 \%)$.

m.p. 180-182 ${ }^{\circ} \mathrm{C} ; R_{\mathrm{f}} 0.5$ (EtOAc:Hex 3:10); $[\alpha]^{24}{ }_{\mathrm{D}}+69.7$ (c 1.82, $\left.\mathrm{CH}_{2} \mathrm{Cl}_{2}\right) ;{ }^{1} \mathrm{H}$ NMR 8.17-8.15 (m, 2H), 8.02-8.00 (m, 2H), 7.62-7.55 (m, 2H), 7.48-7.44 (m, 4H), 7.33-7.16 (m, 10H), 5.85 (d, J = 10.3 Hz, 1H), 5.65 (dd, J = 10.2, 8.1 $\mathrm{Hz} 1 \mathrm{H}$ ), 5.57 (d, J=2.9 Hz, 1H), 5.35 (ddd, $J=10.3,4.7,2.3 \mathrm{~Hz}, 1 \mathrm{H}), 4.97(\mathrm{bs}, 1 \mathrm{H}), 4.95(\mathrm{~d}, J=12.7 \mathrm{~Hz}, 1 \mathrm{H}), 4.69$ $(\mathrm{d}, J=12.7 \mathrm{~Hz}, 1 \mathrm{H}), 4.64(\mathrm{~d}, J=8.0 \mathrm{~Hz}, 1 \mathrm{H}), 4.50(\mathrm{~d}, J=11.7 \mathrm{~Hz}, 1 \mathrm{H}), 4.40(\mathrm{~d}, J=11.7 \mathrm{~Hz}, 1 \mathrm{H}), 4.08(\mathrm{dd}, J=10.2$, $3.5 \mathrm{~Hz}, 1 \mathrm{H}), 3.94-3.90(\mathrm{~m}, 2 \mathrm{H}), 3.60(\mathrm{dd}, J=9.0,1.6 \mathrm{~Hz}, 1 \mathrm{H}), 1.34(\mathrm{~d}, J=6.0 \mathrm{~Hz}, 3 \mathrm{H}), 1.32(\mathrm{~d}, J=6.2 \mathrm{~Hz}, 3 \mathrm{H}) ;{ }^{13} \mathrm{C}$ NMR $(125 \mathrm{~Hz})$ 166.4, 165.3, 138.3, 137.3,133.3, 133.3, 131.5, 130.3, 130.0, 129.8, 128.6, 128.6, 128.5, 128.5, 128.0, 127.9, 127.8, 126.3, 99.8, 97.1, 77.7, 76.1, 73.6, 72.2, 70.4, 70.3, 70.2, 65.9, 18.2, 16.6; IR ( $\mathrm{NaCl}) \vee 2982$, 2871, 1726, 1452, 1267, 1112, 1072, 992, 735, $711 \mathrm{~cm}^{-1}$; HRMS (ESI) m/z: $[\mathrm{M}+\mathrm{Na}]^{+}$Calcd for $\mathrm{C}_{40} \mathrm{H}_{40} \mathrm{NaO}_{9}$ 687.2565; Found 687.2564.

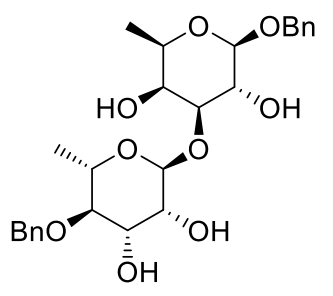


(2S,3R,4S,5R,6S)-5-(benzyloxy)-2-((2R,3R,4S,5S,6R)-2-(benzyloxy)-3,5-dihydroxy-6-methyltetrahydro-2Hpyran-4-yl)oxy)-6-methyltetrahydro-2H-pyran-3,4-diol (23): Following the general procedure, 21a (65 mg, $0.148 \mathrm{mmol})$ in acetone/THF (1:1 ratio by volume, $0.72 \mathrm{~mL}$ ) was reacted with 4-methylmorpholine $\mathrm{N}$-oxide (28.3 mg, $0.242 \mathrm{mmol}$ ), $\mathrm{OsO}_{4}$ solution ( $4 \mathrm{wt} \%$ in $\mathrm{H}_{2} \mathrm{O}, 0.027 \mathrm{~mL}, 4.27 \mu \mathrm{mol}$ ) and distilled $\mathrm{H}_{2} \mathrm{O}(0.09 \mathrm{~mL}, 1.6 \mathrm{M}$ ). Flash column chromatography on silica gel afforded 23 as white solid ( $63.2 \mathrm{mg}, 0.129 \mathrm{mmol}, 90 \%$ yield).

m.p. $192.5-194^{\circ} \mathrm{C} ; R_{\mathrm{f}} 0.2$ (EtOAc:Hex 7:10); $[\alpha]^{24}{ }_{\mathrm{D}}-52.3\left(c \mathrm{c} 0.57, \mathrm{CH}_{2} \mathrm{Cl}_{2}\right.$ ); ${ }^{1} \mathrm{H}$ NMR 7.42-7.39 (m, 4H), 7.35-7.32 $(\mathrm{m}, 4 \mathrm{H}), 7.30-7.26(\mathrm{~m}, 2 \mathrm{H}), 5.0(\mathrm{~s}, 1 \mathrm{H}), 4.95(\mathrm{~d}, J=11.1 \mathrm{~Hz}, 1 \mathrm{H}), 4.89(\mathrm{~d}, J=11.6 \mathrm{~Hz}, 1 \mathrm{H}), 4.89(\mathrm{~s}, 4 \mathrm{H}), 4.65(\mathrm{~d}, J$ $=11.1 \mathrm{~Hz}, 1 \mathrm{H}), 4.63(\mathrm{~d}, J=11.6 \mathrm{~Hz}, 1 \mathrm{H}), 4.34(\mathrm{~d}, J=7.7 \mathrm{~Hz}, 1 \mathrm{H}), 4.01-3.99(\mathrm{~m}, 2 \mathrm{H}), 3.90-3.84(\mathrm{~m}, 1 \mathrm{H}), 3.71-3.62$ (m, 3H), $3.55(\mathrm{dd}, J=9.9,3.2 \mathrm{~Hz}, 1 \mathrm{H}), 3.44-3.40(\mathrm{~m}, 1 \mathrm{H}), 1.29(\mathrm{~d}, J=6.4 \mathrm{~Hz}, 3 \mathrm{H}), 1.26(\mathrm{~d}, J=6.3 \mathrm{~Hz}, 3 \mathrm{H}) ;{ }^{13} \mathrm{C}$ NMR (125 Hz) 140.1, 139.1, 129.2, 129.2, 129.1, 129.1, 128.6, 103.8, 103.8, 82.6, 81.8, 76.0, 72.8, 72.6, 72.5, 71.9, 71.8, 71.7, 69.1, 18.3, 16.7; IR (NaCl) v 3490, 2935, 2909, 1454, 1377, 1169, 1070, 1048, 732, $695 \mathrm{~cm}^{-1}$; HRMS (ESI) $\mathrm{m} / \mathrm{z}:[\mathrm{M}+\mathrm{Na}]^{+}$Calcd for $\mathrm{C}_{26} \mathrm{H}_{34} \mathrm{NaO}_{9}$ 513.2095; Found 513.2096.

3) synthesis of $\mathbf{S 8}$

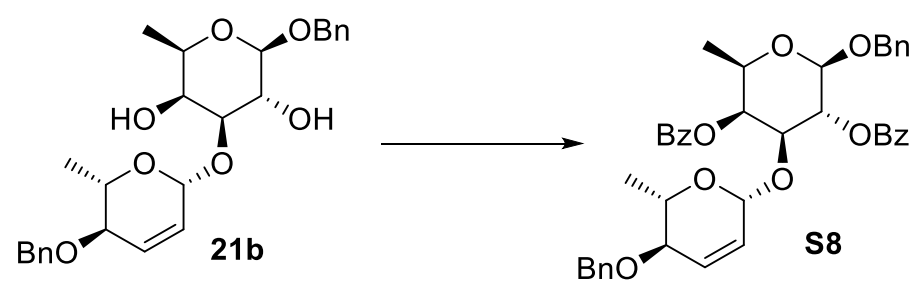

(2R,3R,4S,5S,6R)-2-(benzyloxy)-4-(((2R,5R,6S)-5-(benzyloxy)-6-methyl-5,6-dihydro-2H-pyran-2-yl)oxy)-6methyltetrahydro-2H-pyran-3,5-diyl dibenzoate (S8): To a solution of 21b (34 mg, $0.074 \mathrm{mmol}$ ), in pyridine $(0.4 \mathrm{~mL})$, was added benzoyl chloride $(19 \mu \mathrm{L}, 0.164 \mathrm{mmol})$ at $0{ }^{\circ} \mathrm{C}$. The reaction mixture was stirred at room temperature for $12 \mathrm{~h}$ (monitored by TLC). The reaction was quenched with water and extracted with ethyl acetate $(5 \mathrm{~mL})$. The organic layer was washed with water $(3 \times 5 \mathrm{~mL})$, dried over sodium sulfate, concentrated under reduced pressure to obtain crude. The crude was purified by column chromatography (eluent: hexane/EtOAc 70:30) on silica gel to afford $\mathbf{S 8}$ as white solid (39.9 mg, $0.062 \mathrm{mmol}, 84 \%$ ).

m.p. $140-145^{\circ} \mathrm{C} ; R_{\mathrm{f}} 0.5$ (EtOAc:Hex 3:10); $[\alpha]^{24}{ }_{\mathrm{D}}+15.4\left(\mathrm{c} 0.61, \mathrm{CH}_{2} \mathrm{Cl}_{2}\right) ;{ }^{1} \mathrm{H}$ NMR 8.19-8.17 (m, 2H), 8.02-8.01 (m, 2H), 7.61-7.54 (m, 2H), 7.49-7.47(m, 2H), 7.44-7.40 (m, 2H), 7.33-7.15 (m, 10H), $5.87(\mathrm{~d}, J=10.4 \mathrm{~Hz}, 1 \mathrm{H}), 5.59$ $(\mathrm{d}, J=3.1 \mathrm{~Hz}, 1 \mathrm{H}), 5.54(\mathrm{~d}, J=10.4 \mathrm{~Hz}, 1 \mathrm{H}), 5.50(\mathrm{dd}, J=10.0,8.1 \mathrm{~Hz} 1 \mathrm{H}), 5.29(\mathrm{~d}, J=1.6 \mathrm{~Hz} 1 \mathrm{H}), 4.94(\mathrm{~d}, J=$ 12.7 Hz, 1H), $4.70(\mathrm{~d}, J=12.8 \mathrm{~Hz}, 1 \mathrm{H}), 4.68(\mathrm{~d}, J=8.1 \mathrm{~Hz}, 1 \mathrm{H}), 4.52(\mathrm{~d}, J=11.7 \mathrm{~Hz}, 1 \mathrm{H}), 4.42(\mathrm{~d}, J=11.7 \mathrm{~Hz}, 1 \mathrm{H})$, $4.27(\mathrm{dd}, J=10.1,3.5 \mathrm{~Hz}, 1 \mathrm{H}), 3.89-3.85(\mathrm{~m}, 1 \mathrm{H}), 3.74-3.69(\mathrm{~m}, 1 \mathrm{H}), 3.42-3.40(\mathrm{~m}, 1 \mathrm{H}), 1.36(\mathrm{~d}, J=6.4 \mathrm{~Hz}, 3 \mathrm{H})$, 0.99 (d, $J=6.4 \mathrm{~Hz}, 3 \mathrm{H}) ;{ }^{13} \mathrm{C}$ NMR $(125 \mathrm{~Hz})$ 166.6, 165.6, 138.3, 137.4,133.4, 132.9, 130.7, 130.3, 130.1, 129.8, 129.0, 128.8, 128.7, 128.6, 128.4, 128.3, 127.9, 127.9, 127.8, 99.9, 93.7, 74.5, 74.5, 72.2, 71.0, 70.9, 70.6, 70.3, 
69.8, 18.9, 16.7; IR (NaCl) v 2982, 2869, 1724, 1452, 1268, 1110, 1071, 1027, 736, $711 \mathrm{~cm}^{-1}$; HRMS (ESI) m/z: $[\mathrm{M}+\mathrm{Na}]^{+}$Calcd for $\mathrm{C}_{40} \mathrm{H}_{40} \mathrm{NaO} 9687.2565$; Found 687.2565.

8. Structure determination of compound $7 \mathrm{~b}$ by $1 \mathrm{H}$ NMR and 2D NMR of dihydroxylated product S9

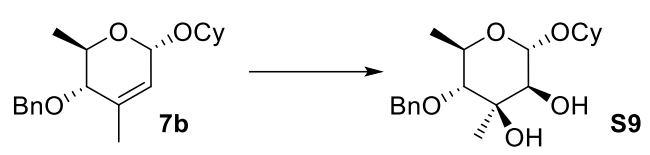

(2S,3S,4R,5R,6R)-5-(benzyloxy)-2-(cyclohexyloxy)-4,6-dimethyltetrahydro-2H-pyran-3,4-diol (S9): Following the general procedure, $7 \mathrm{~b}(60.0 \mathrm{mg}, 0.190 \mathrm{mmol})$ in acetone/THF (1:1 ratio by volume, $0.20 \mathrm{~mL})$ was reacted with 4-methylmorpholine $\mathrm{N}$-oxide $\left(44.0 \mathrm{mg}, 0.380 \mathrm{mmol}\right.$ ), $\mathrm{OsO}_{4}$ solution (4 wt \% in $\mathrm{H}_{2} \mathrm{O}, 60.0 \mu \mathrm{L}, 9.5 \mu \mathrm{mol}$ ) and distilled $\mathrm{H}_{2} \mathrm{O}(0.12 \mathrm{~mL}, 1.6 \mathrm{M})$. Flash column chromatography on silica gel afforded $\mathbf{S} 9$ as a colorless oil (48.0 $\mathrm{mg}, 0.136 \mathrm{mmol}, 71 \%$ yield).

$R_{\mathrm{f}} 0.50$ (EtOAc:Hex 5:5); $[\alpha]^{25}{ }_{\mathrm{D}}+80.1\left(c 0.91, \mathrm{CHCl}_{3}\right) ;{ }^{1} \mathrm{H} \mathrm{NMR}\left(300 \mathrm{MHz}, \mathrm{CDCl}_{3}\right) \delta$ 7.37-7.30 (m, 5H), $4.92(\mathrm{~s}, 1 \mathrm{H})$, $4.83(\mathrm{~d}, J=11.5 \mathrm{~Hz}, 1 \mathrm{H}), 4.71(\mathrm{~d}, J=11.5 \mathrm{~Hz}, 1 \mathrm{H}), 3.76(\mathrm{dq}, J=9.6,6.2 \mathrm{~Hz}, 1 \mathrm{H}), 3.63-3.58(\mathrm{~m}, 1 \mathrm{H}), 3.52(\mathrm{~d}, J=$ $3.3 \mathrm{~Hz}, 1 \mathrm{H}), 3.31(\mathrm{~d}, J=9.6 \mathrm{~Hz}, 1 \mathrm{H}), 2.57(\mathrm{~d}, J=4.3 \mathrm{~Hz}, 1 \mathrm{H}), 2.34(\mathrm{~s}, 1 \mathrm{H}), 1.78-1.75(\mathrm{~m}, 2 \mathrm{H}), 1.69-1.68(\mathrm{~m}, 2 \mathrm{H})$, 1.49-1.26 (m, 12H); ${ }^{13} \mathrm{C}$ NMR $\left(75 \mathrm{MHz}, \mathrm{CDCl}_{3}\right) \delta$ 138.7, 128.7, 128.2, 128.0, 97.8, 83.9, 76.2, 75.6, 75.0, 74.2, $66.9,33.4,31.4,25.9,24.0,23.7,20.1$, 18.4; IR (NaCl) v 3397, 2932, 2858, 1451, 1380, 1307, 1134, 1115, 1081 , $1064 \mathrm{~cm}^{-1}$; HRMS (ESI) m/z: [M+Na] ${ }^{+}$Calcd for $\mathrm{C}_{20} \mathrm{H}_{30} \mathrm{NaO}_{5}$ 373.1985; Found 373.1988. 


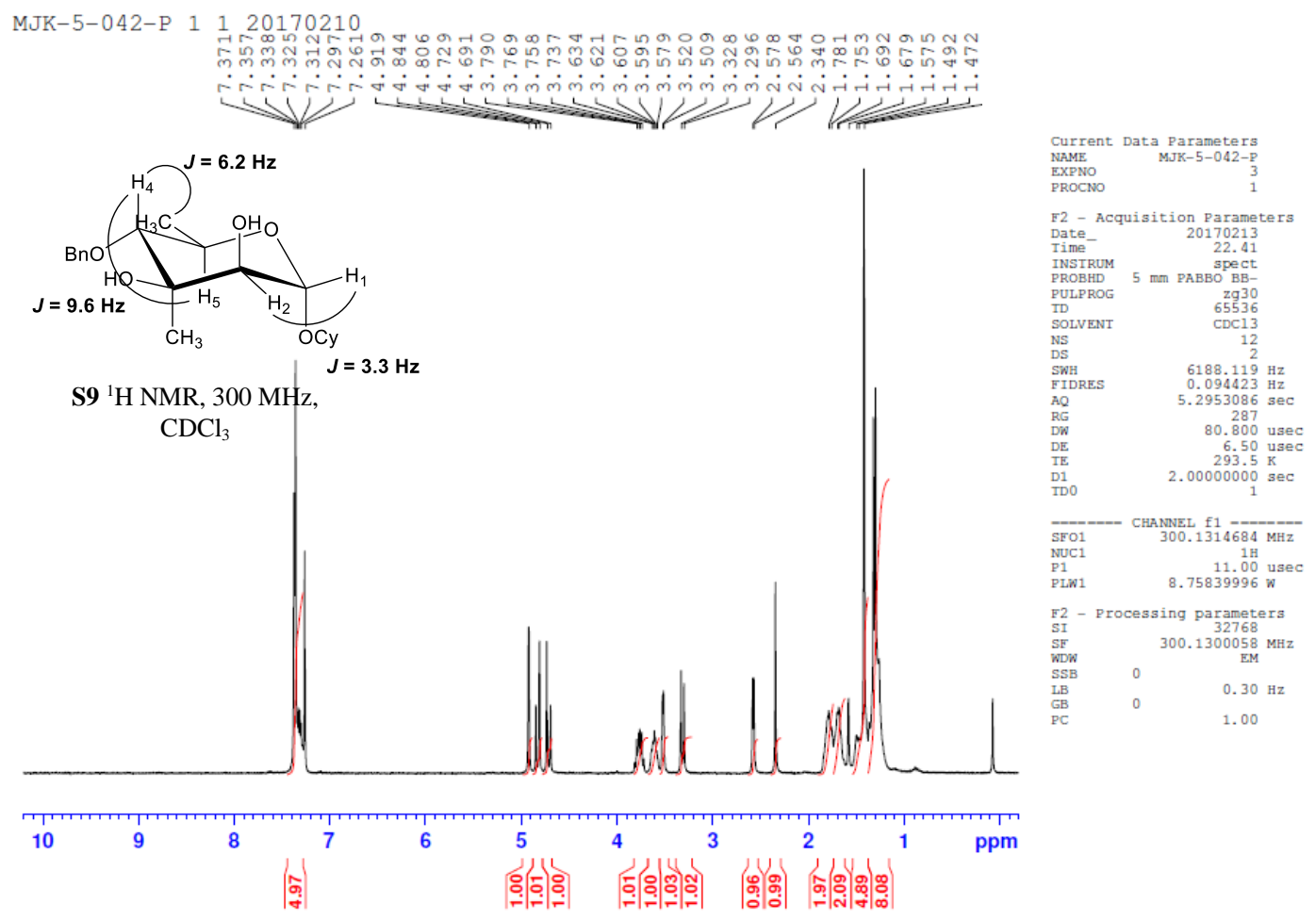

\section{NOESY}
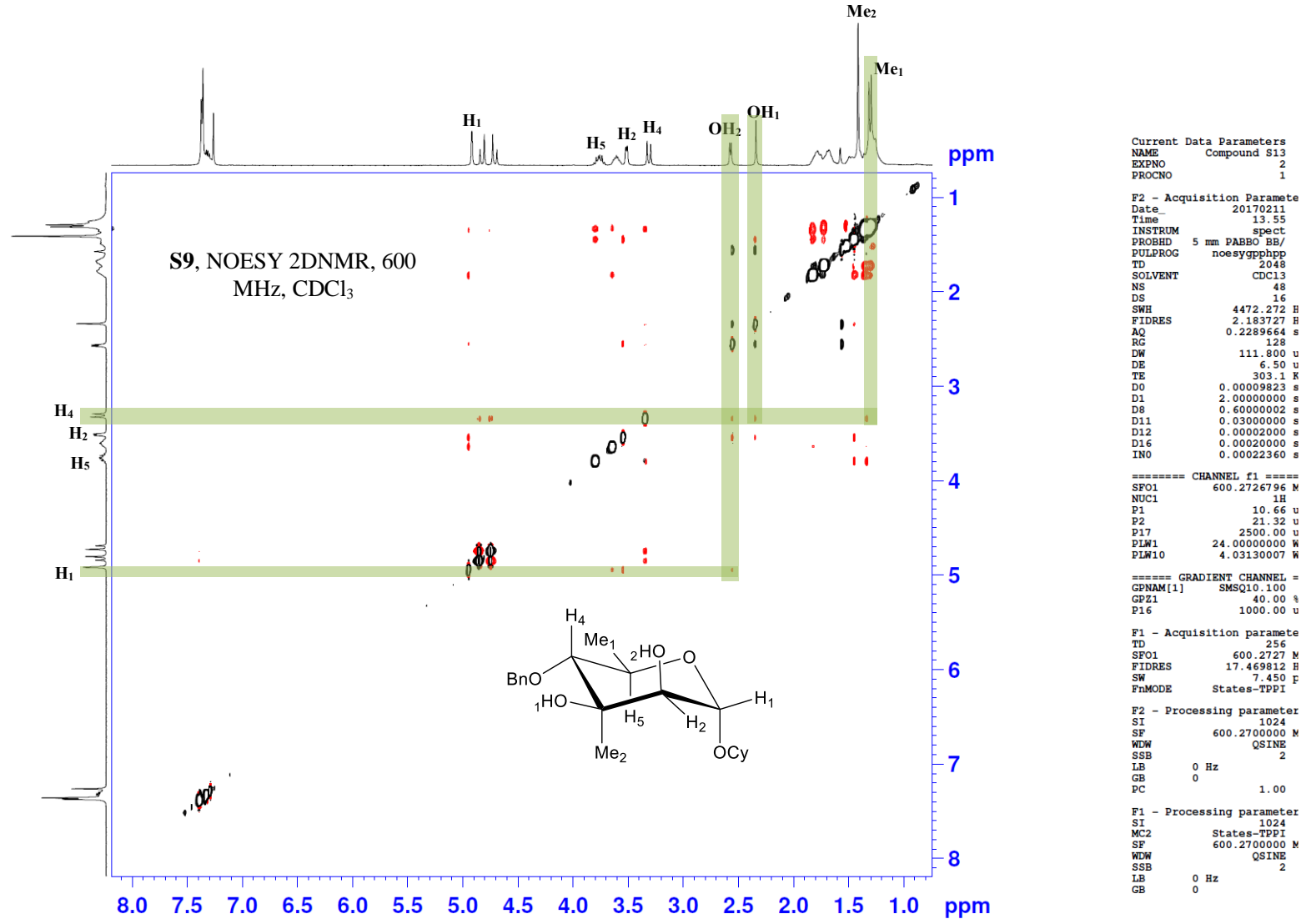


\section{Structure determination of compound S8 and 22 by 2D NMR}

HMBC

BBP-3-082-P-011419
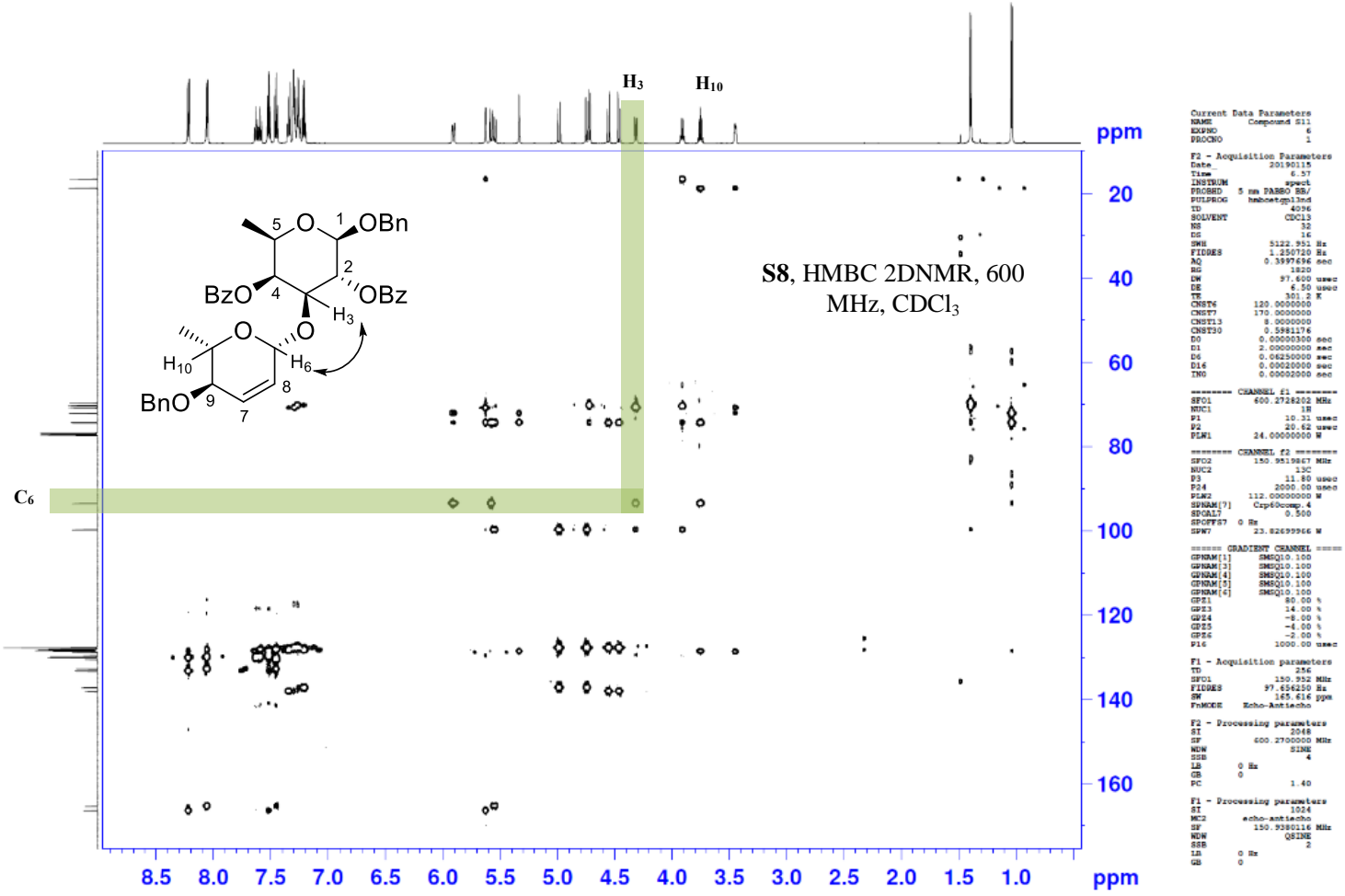

NOESY

BBP-3-082-P-011419
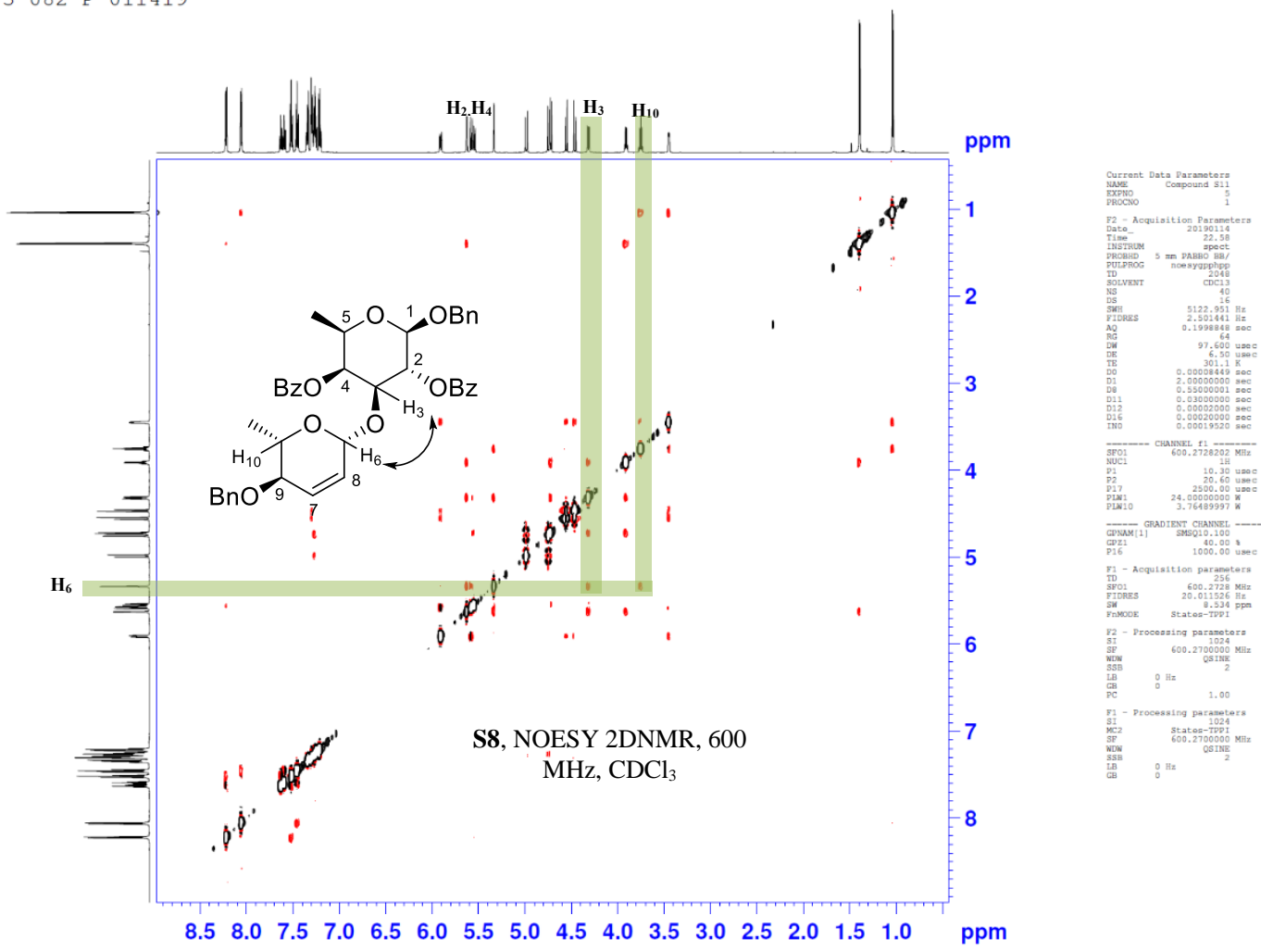
HMBC

BBP-3-083-P-011519
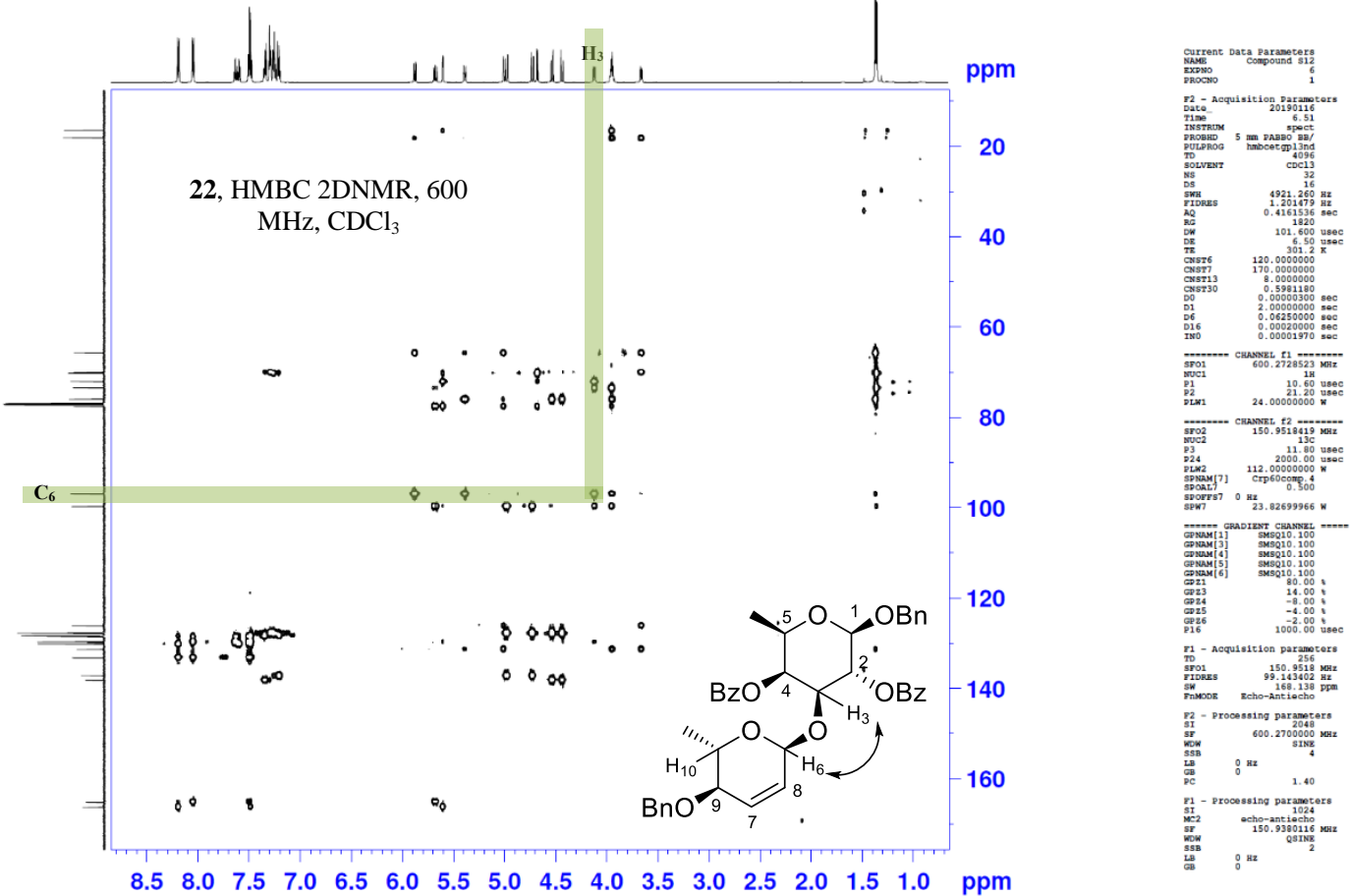

NOESY
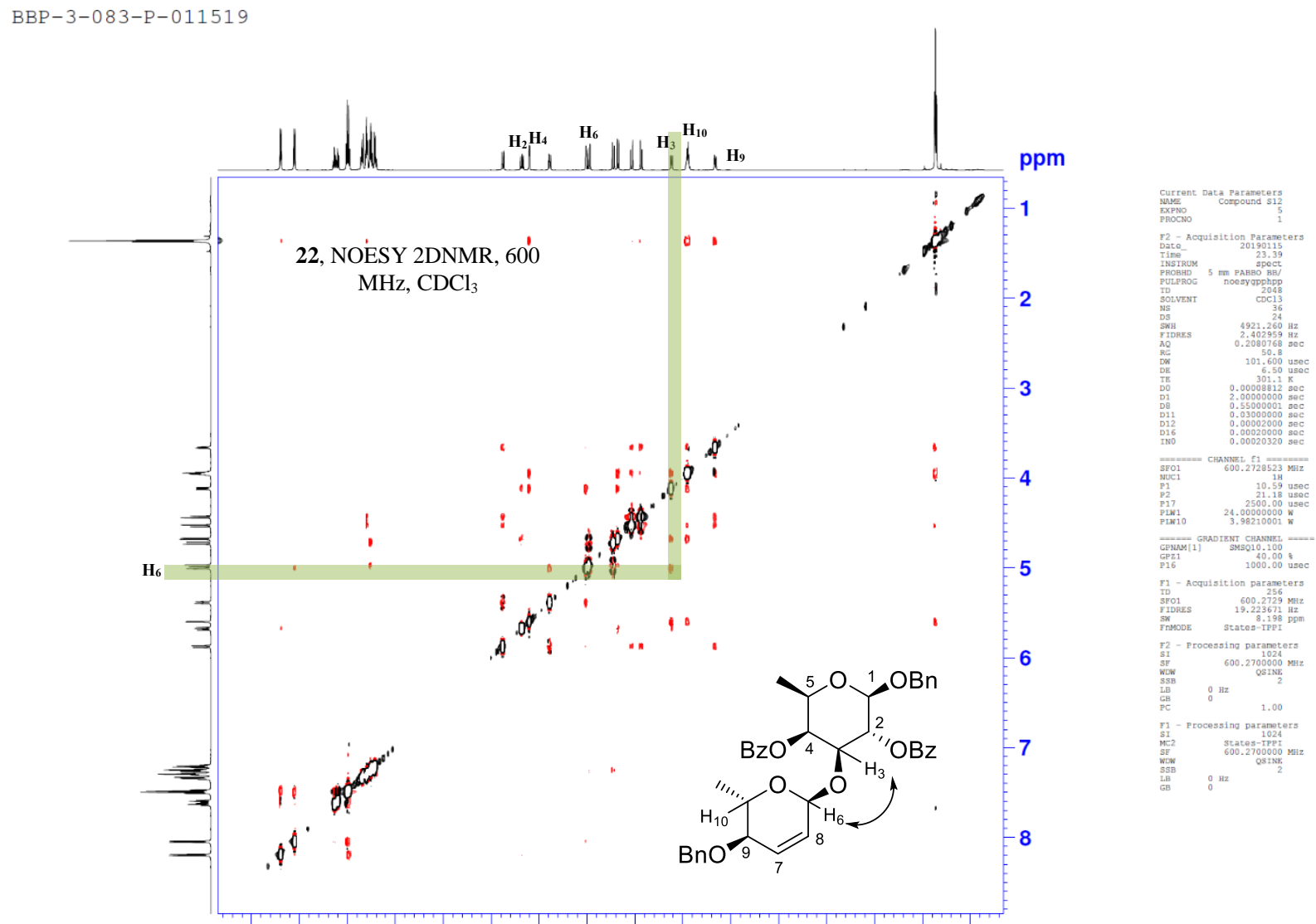

$\begin{array}{llllllllllllllllll}8.5 & 8.0 & 7.5 & 7.0 & 6.5 & 6.0 & 5.5 & 5.0 & 4.5 & 4.0 & 3.5 & 3.0 & 2.5 & 2.0 & 1.5 & 1.0 & \text { ppm }\end{array}$ 


\section{References}

[1] K. R. Prasad, P. Gutala, Tetrahedron 2011, 67, 4514-4520.

[2] T. Amaya, D. Takahashi, H. Tanaka, T. Takahashi, Angew. Chem. Int. Ed. 2003, 42, 1833-1836.

[3] J. Lee, S. Kang, J. Kim, D. Moon, Y. H. Rhee, Angew. Chem. Int. Ed. 2019, 58, 628-631.

[4] C. Li, B. Yu, M. Liu, Y. Hui, Carbohydr. Res. 1967, 4, 189-195 


\section{Ratios of acyclic and cyclic 0,0 -acetals; determined by crude ${ }^{1} \mathrm{H}$ NMR}

BBP-4-236-Cr 1120210610 (65mg mes)

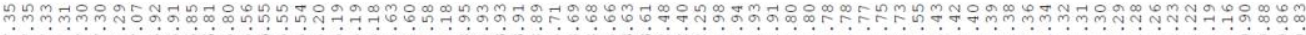

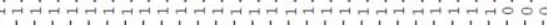
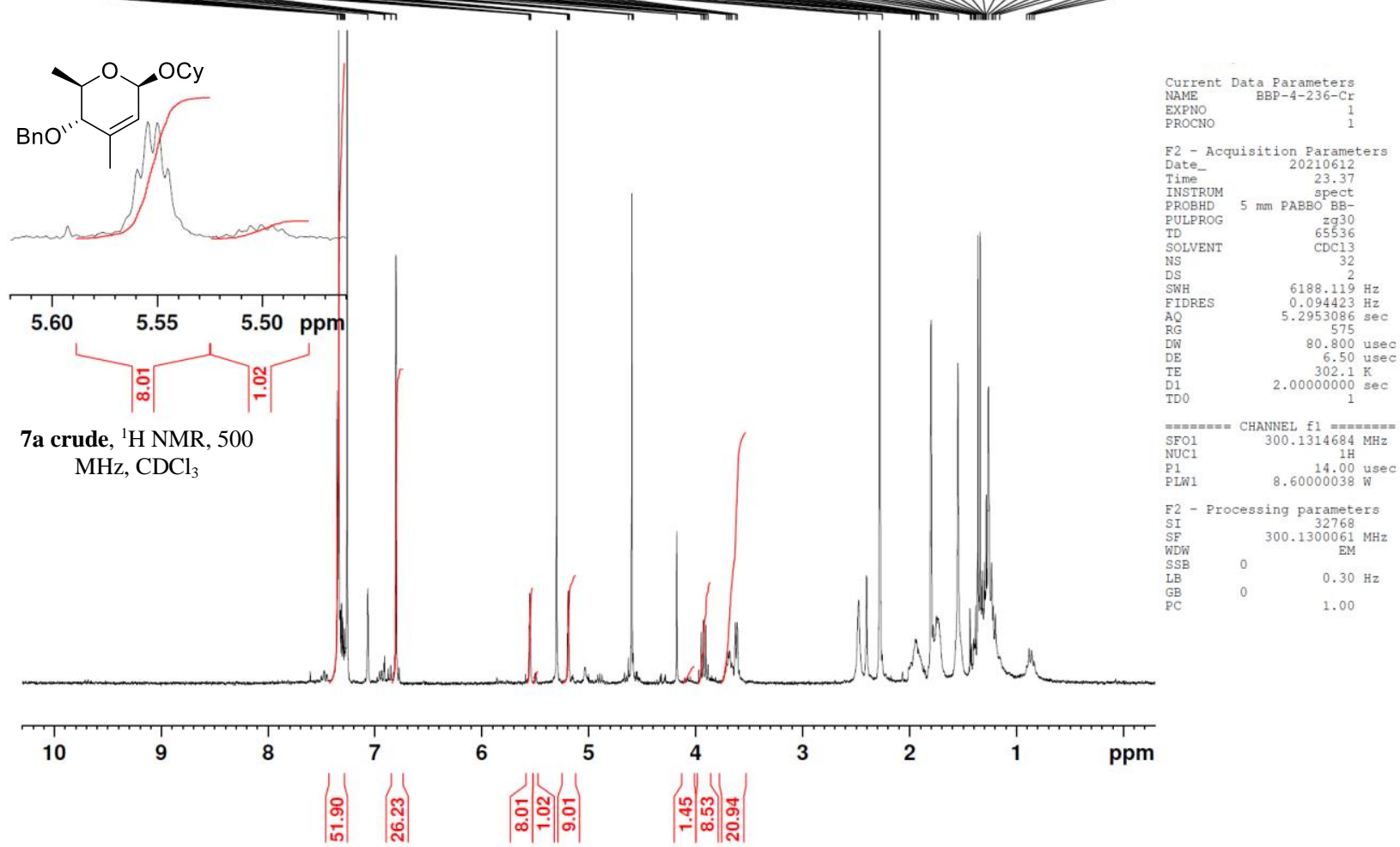

MJK-5-040-CP 11 yhr 20170208

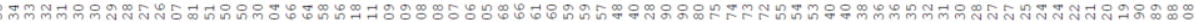

rincring

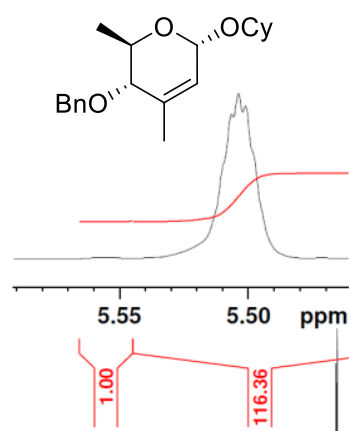

7b crude, ${ }^{1} \mathrm{H} \mathrm{NMR}, 500$ $\mathrm{MHz}, \mathrm{CDCl}_{3}$
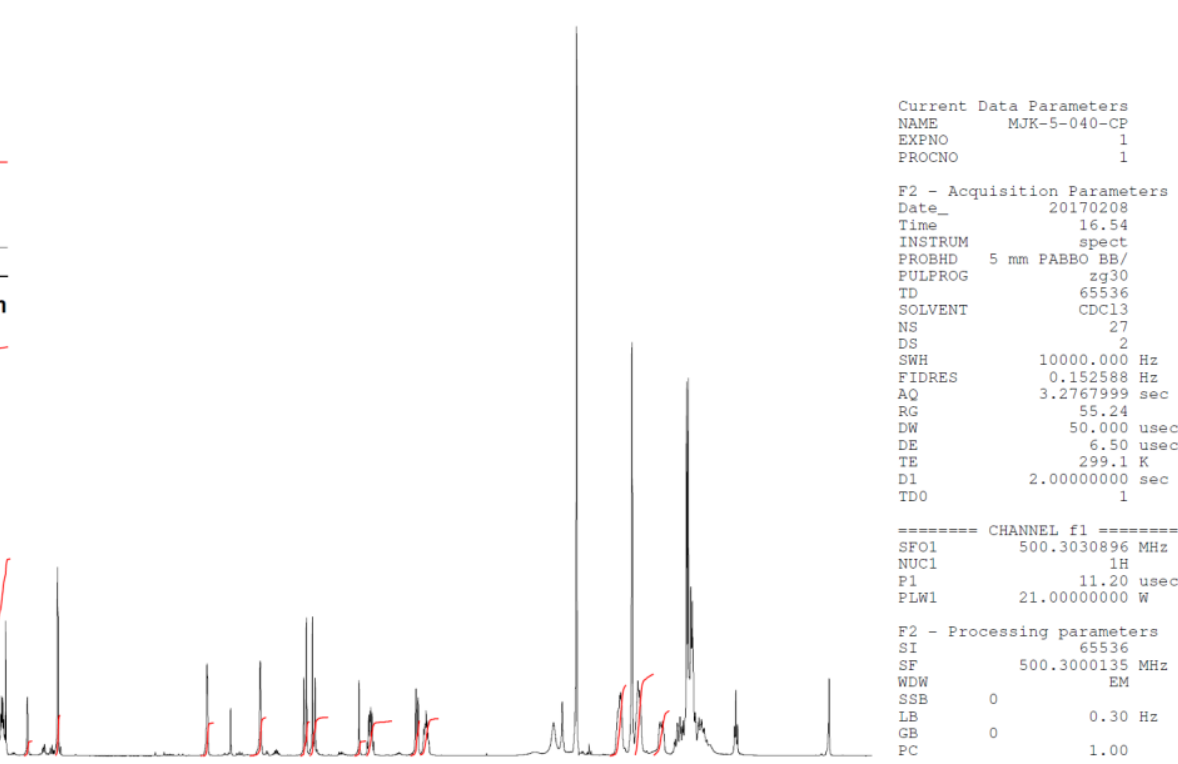

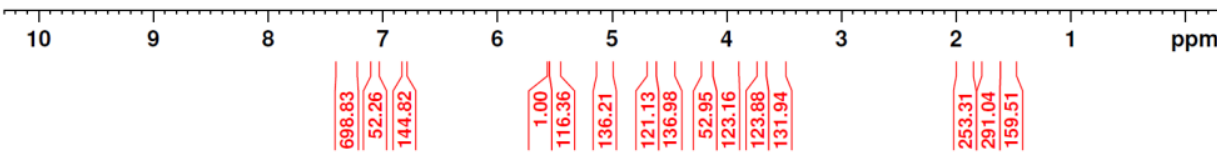




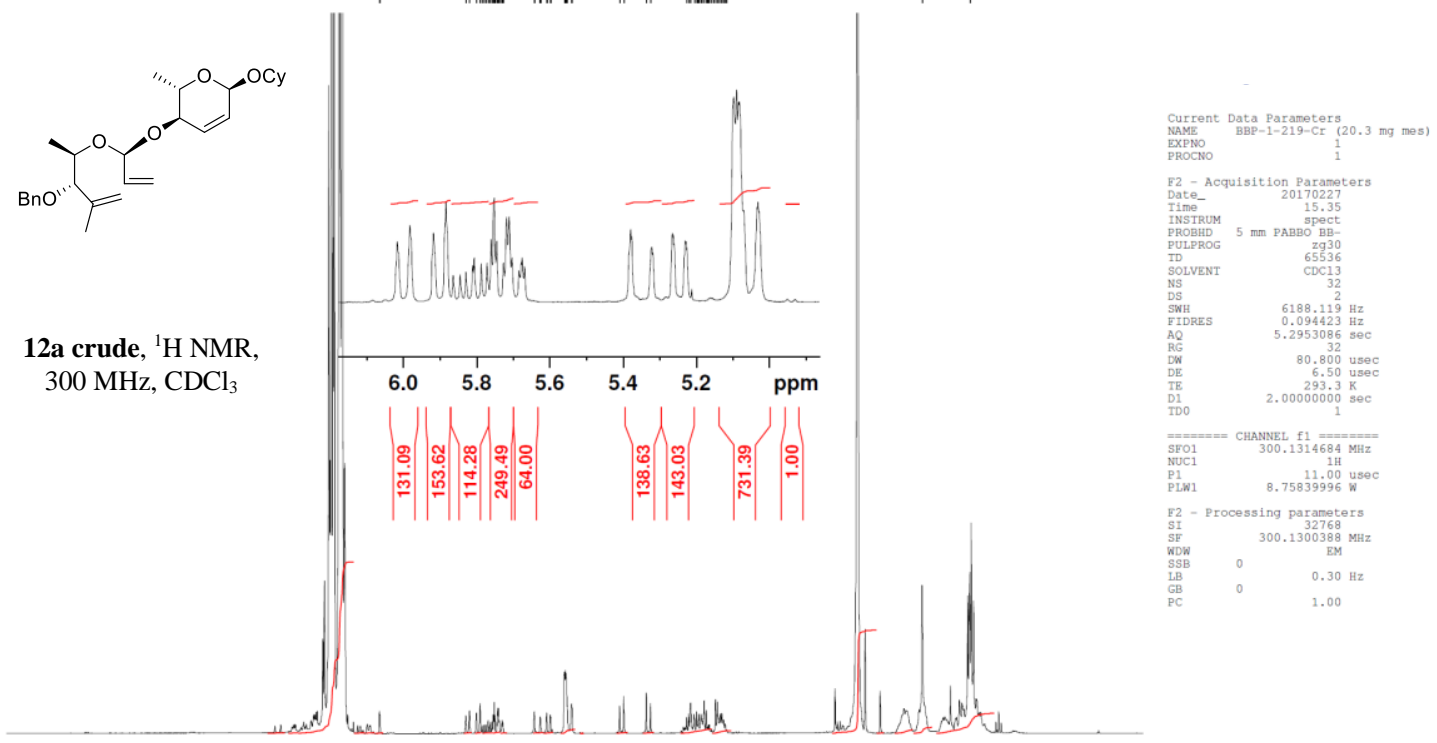

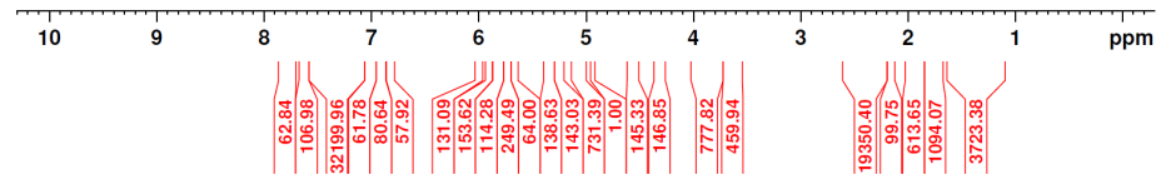

BBP-1-207-Cr (10.3 mg mes) 1120170213

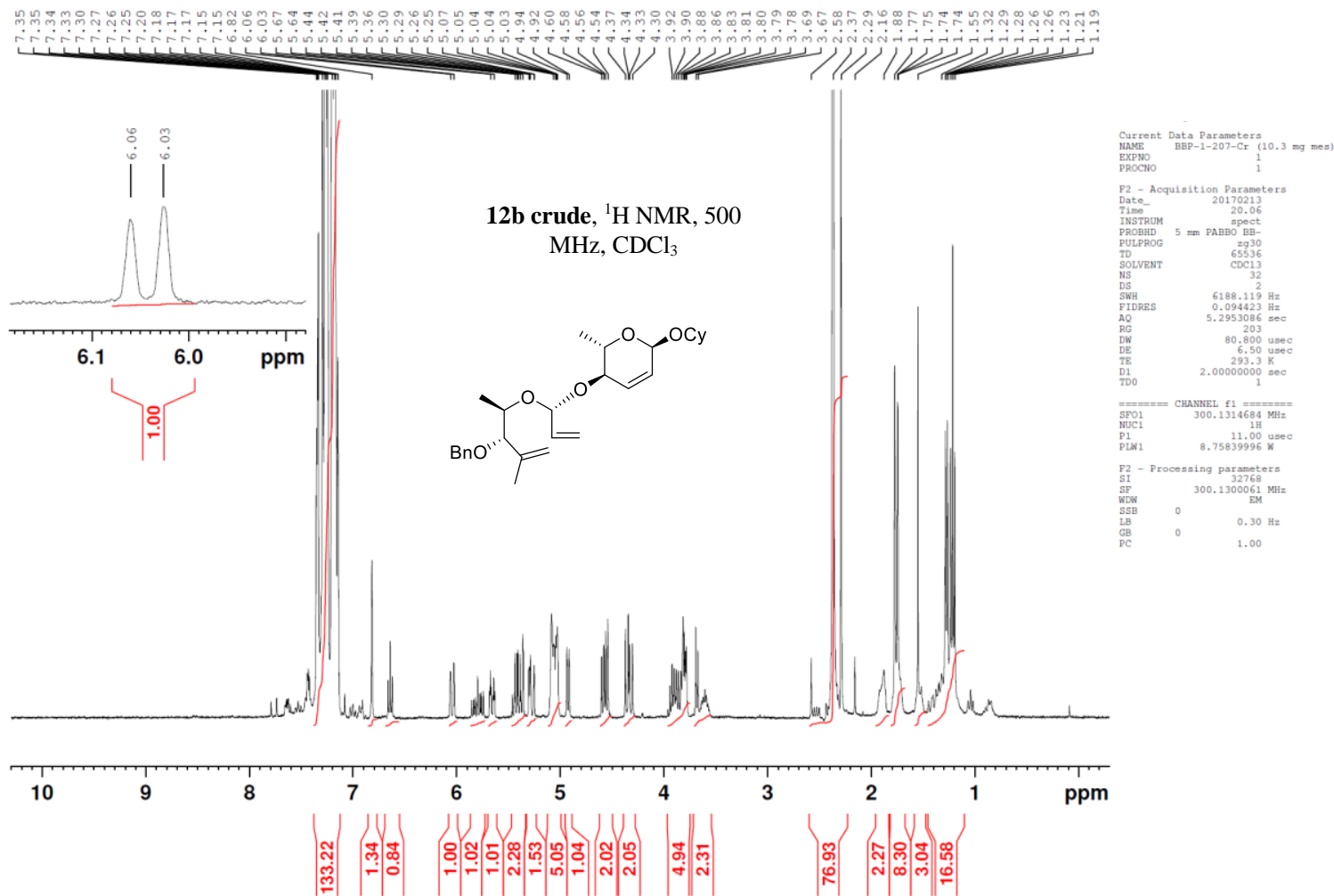


BBP-3-072-Cr 1120181226 (11.1 mg mes)
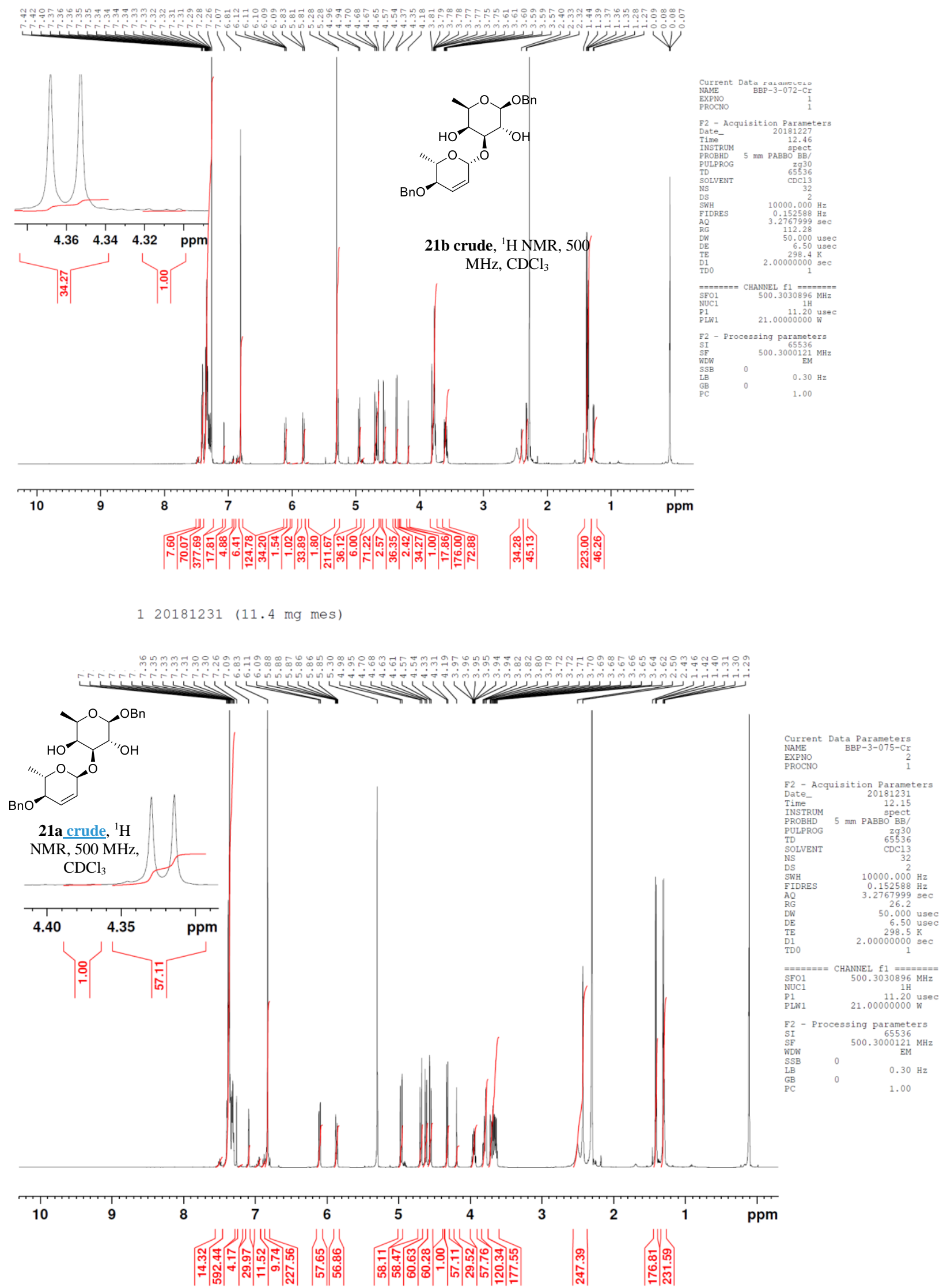


\section{2. ${ }^{1} \mathrm{H}$ and ${ }^{13} \mathrm{C}$ NMR of new compounds}

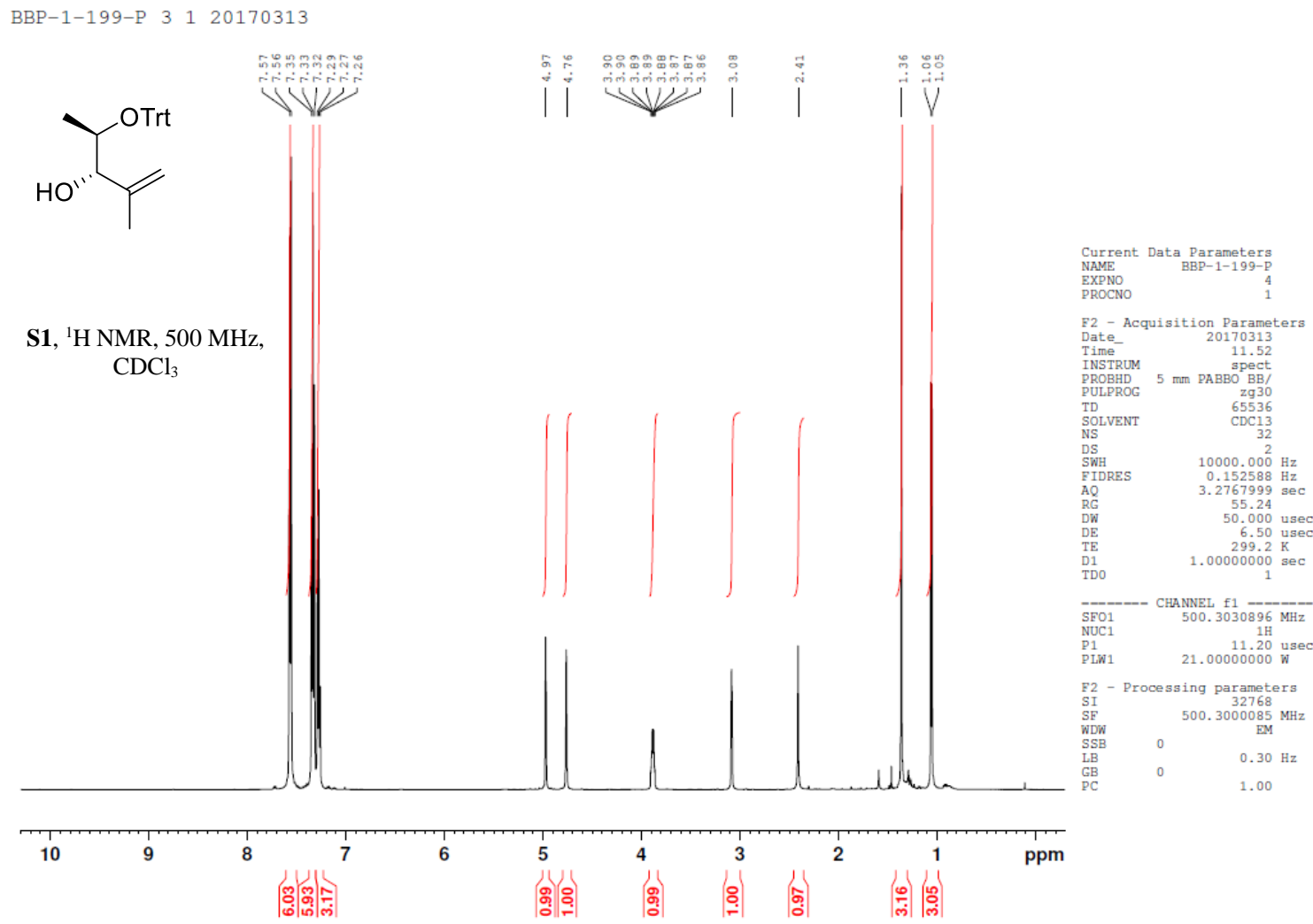

BBP-1-199-P 13120170313
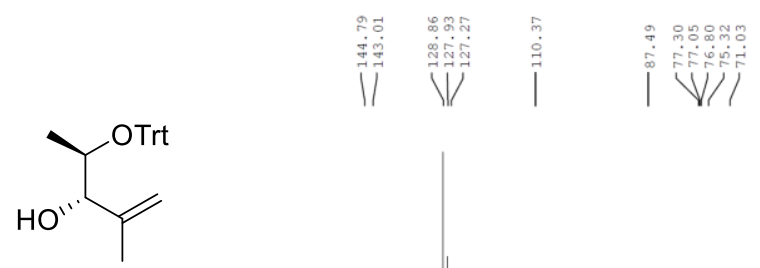

|

S1, ${ }^{13} \mathrm{C}$ NMR, $125 \mathrm{MHz}$, $\mathrm{CDCl}_{3}$
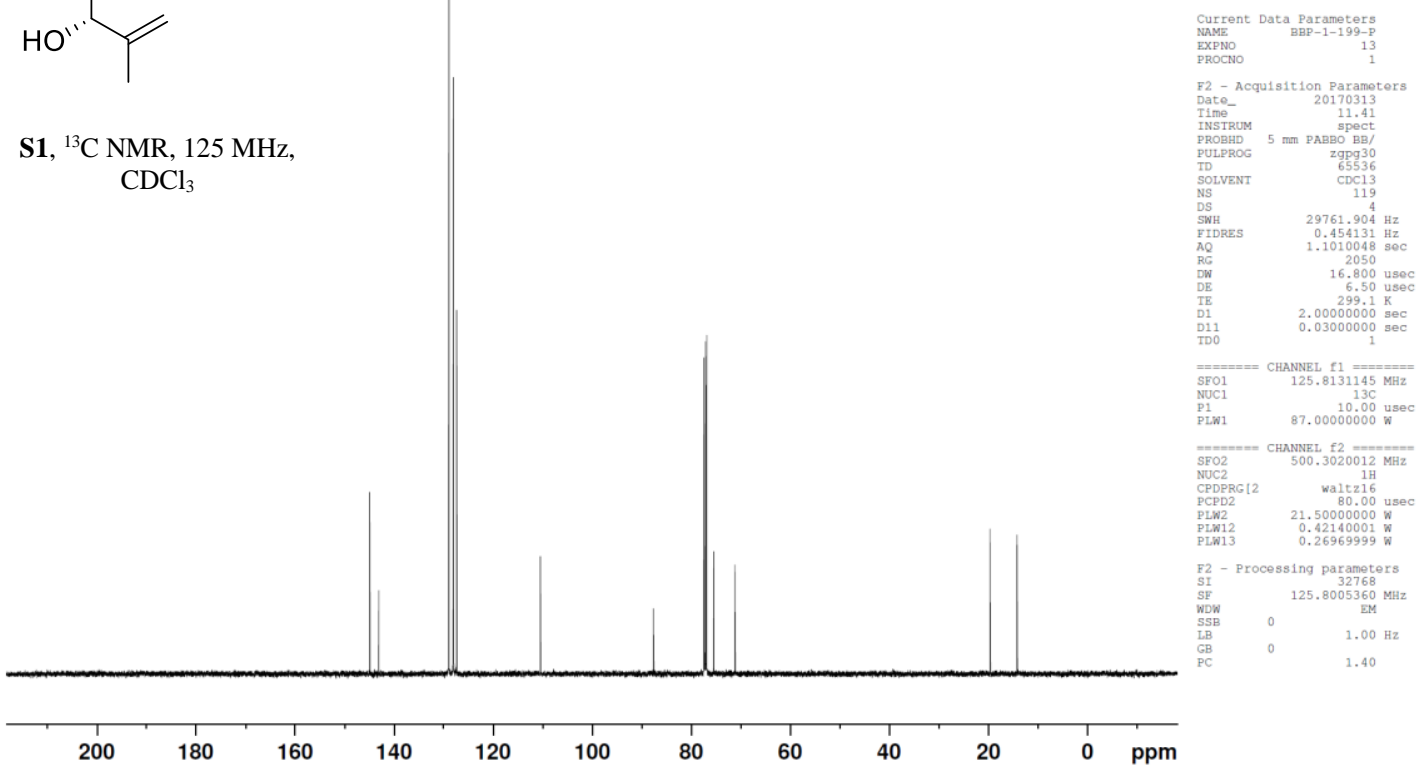


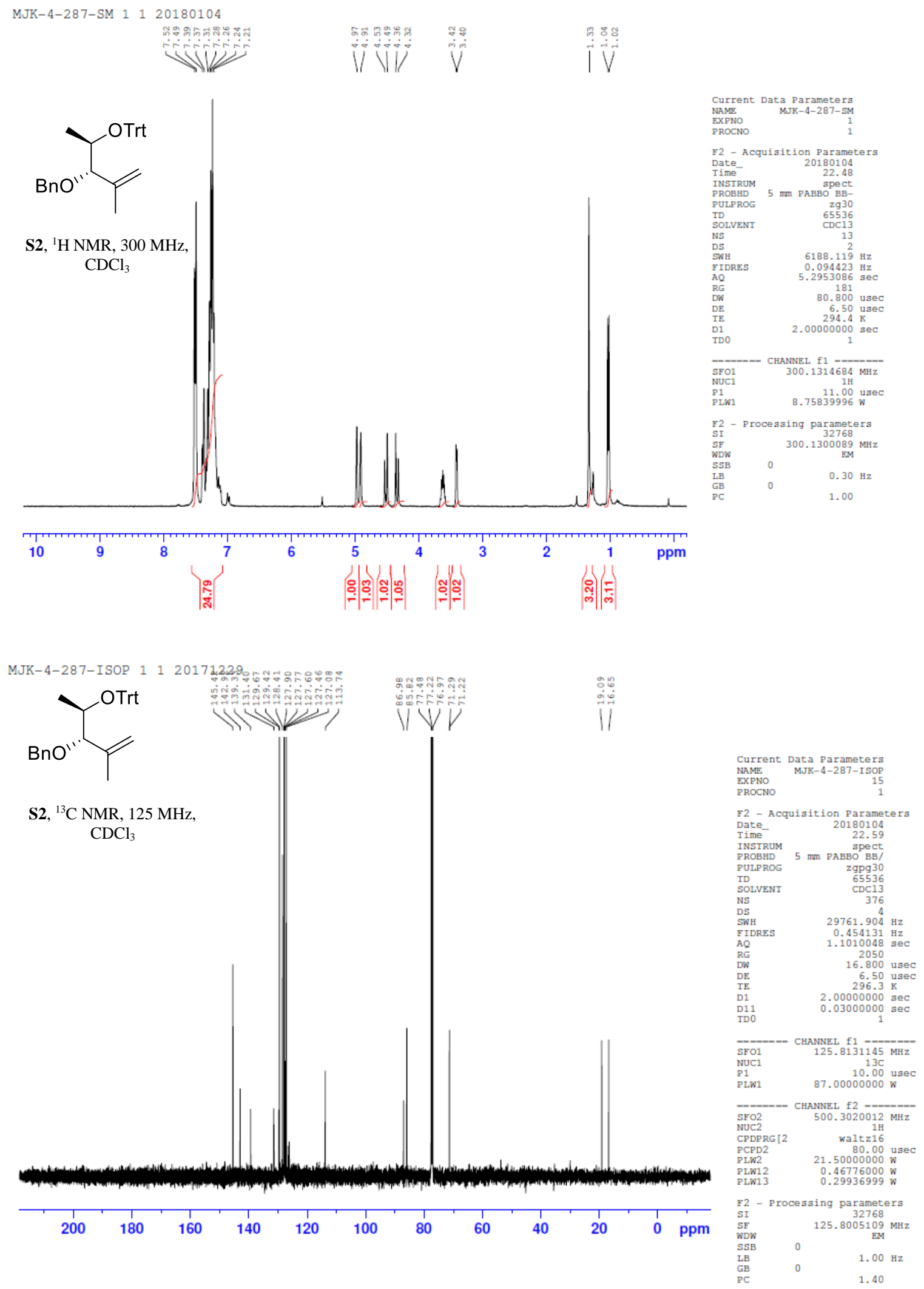




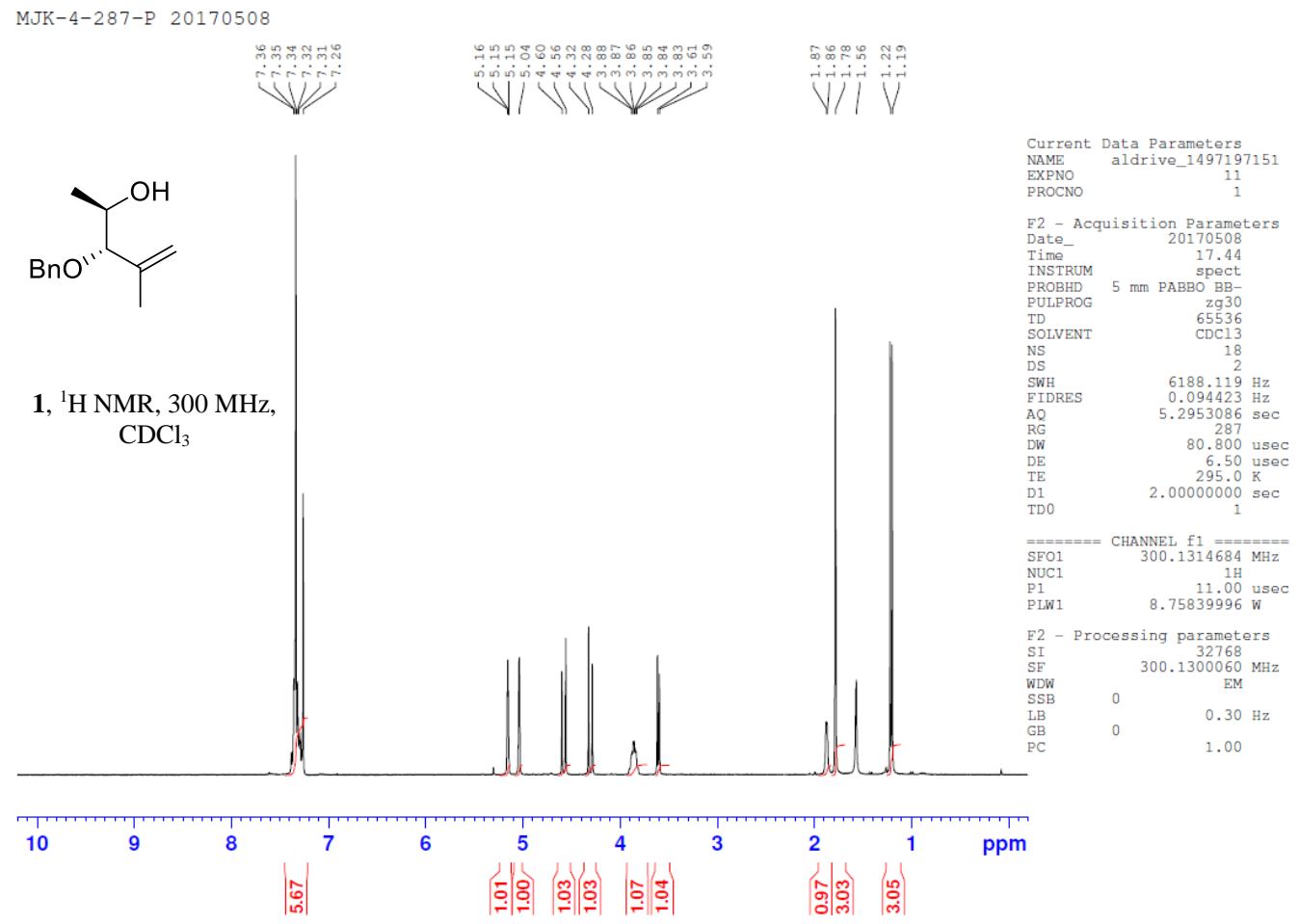

MJK-4-287-P 131 yhr 20161223
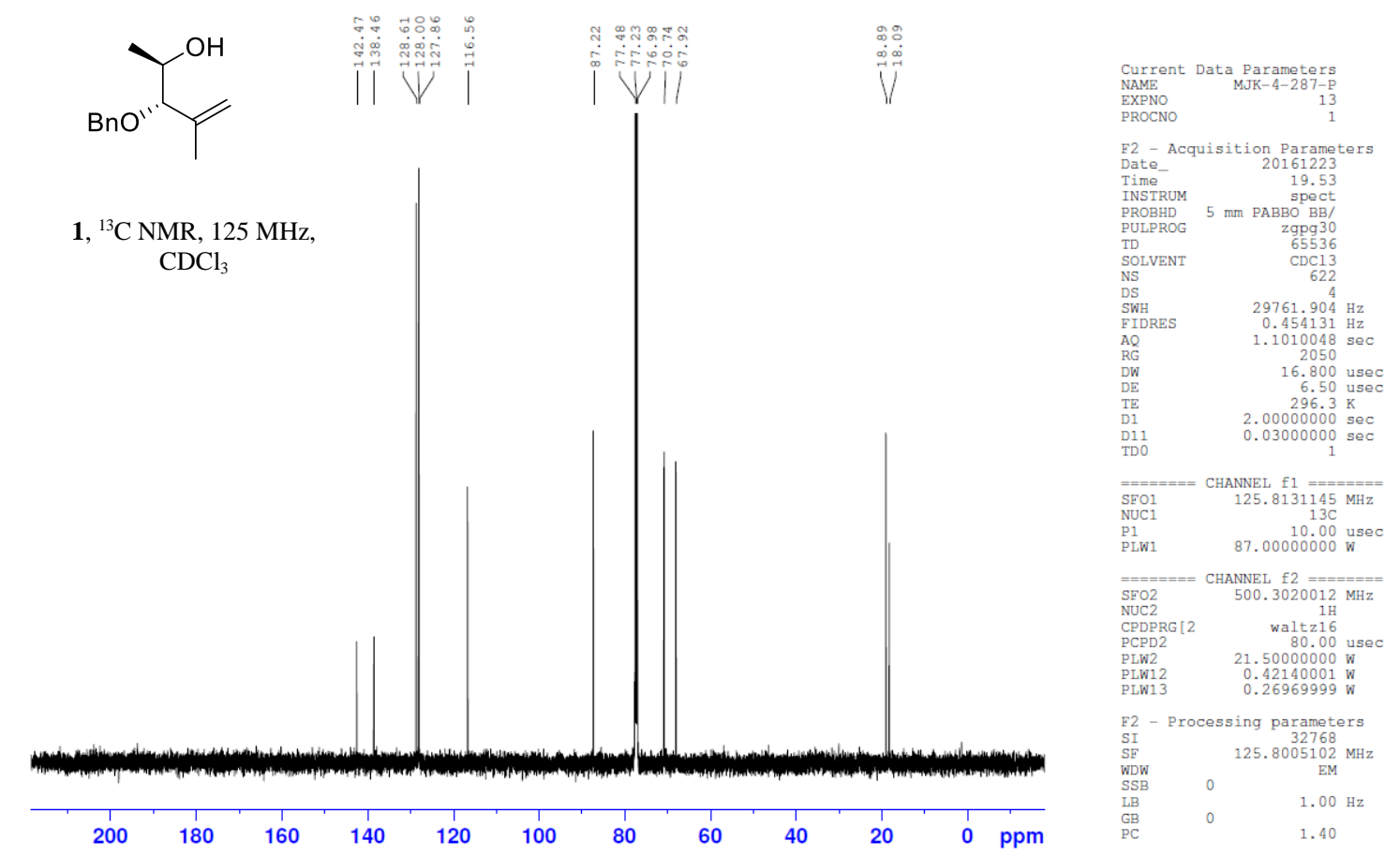


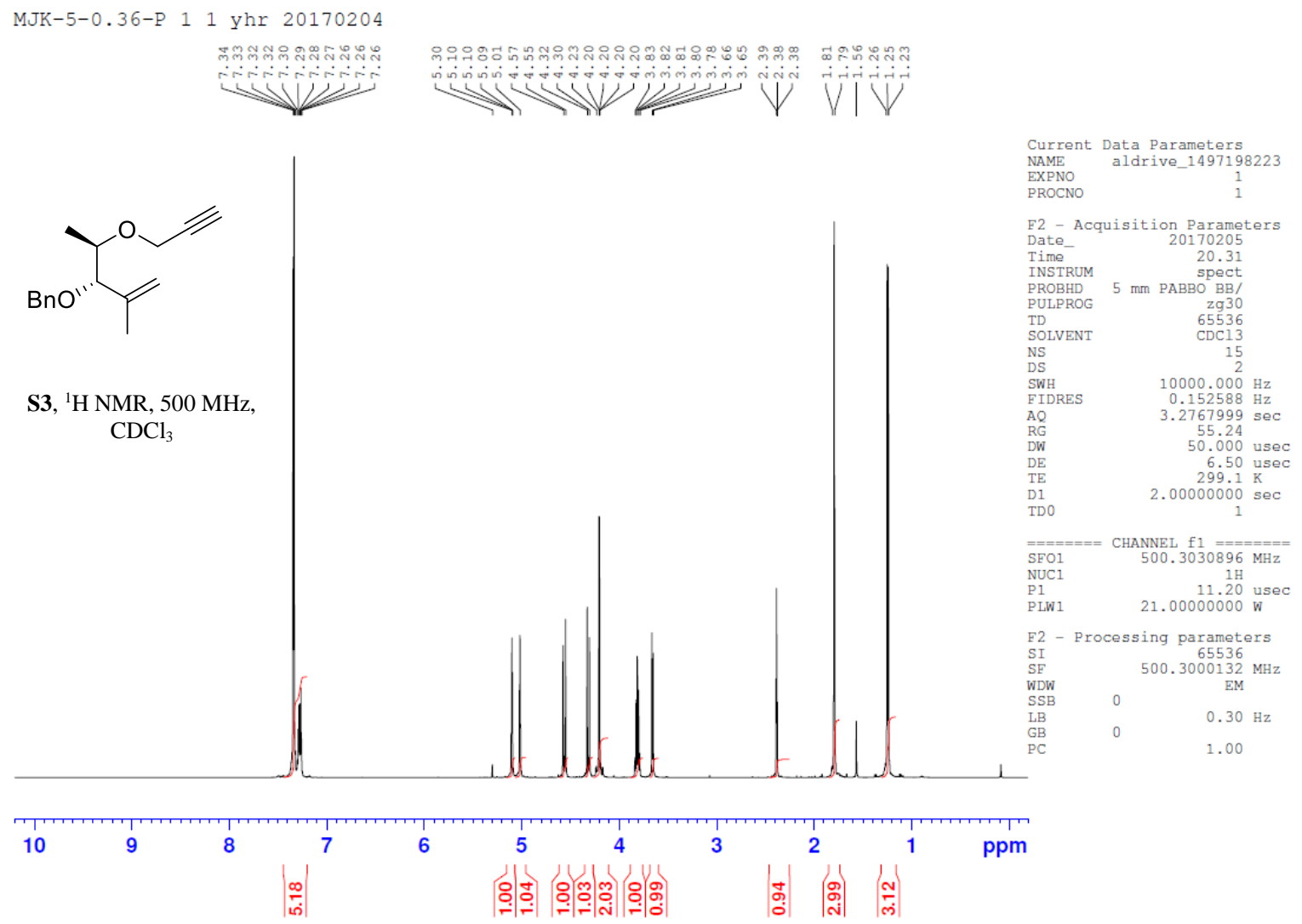

MJK-5-0.36-P 11 yhr 20170204

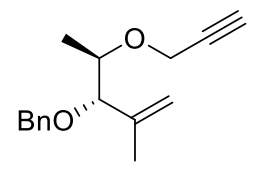

S3, ${ }^{13} \mathrm{C}$ NMR, $125 \mathrm{MHz}$,

$\mathrm{CDCl}_{3}$
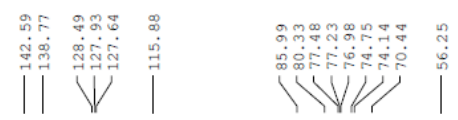

\section{|}

Current Data Parameters EXPNO

F2 - Acquisition Parameter

Time- 20.31

PROBHD $5 \mathrm{~mm}$ PABBO BB/

PULPROG $\mathrm{zg} 30$

SOLVENT

SWH $\quad 10000.000$ Hz

$\mathrm{AQ} \quad 3.2767999 \mathrm{sec}$

DW $\quad 50.000$ usec

$2.00000000 \mathrm{sec}$

500.3030896 MHz

11.20 use

F2 - Processing parameters

SI 65536

SSB $\quad 0=0.30$

$\begin{array}{ll}\mathrm{SSB} & 0.30 \mathrm{~Hz} \\ \mathrm{LB} & 0.00\end{array}$ 

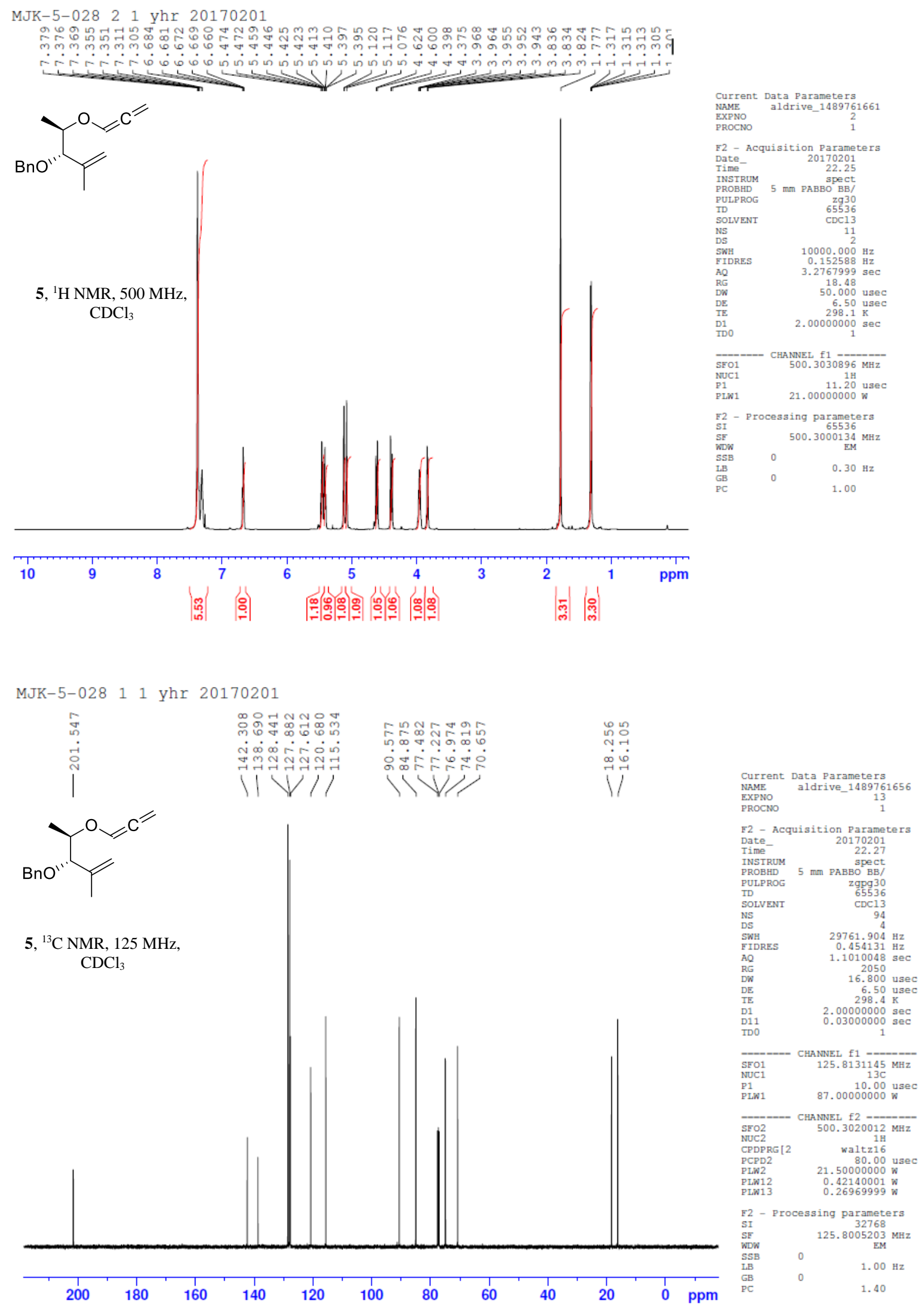

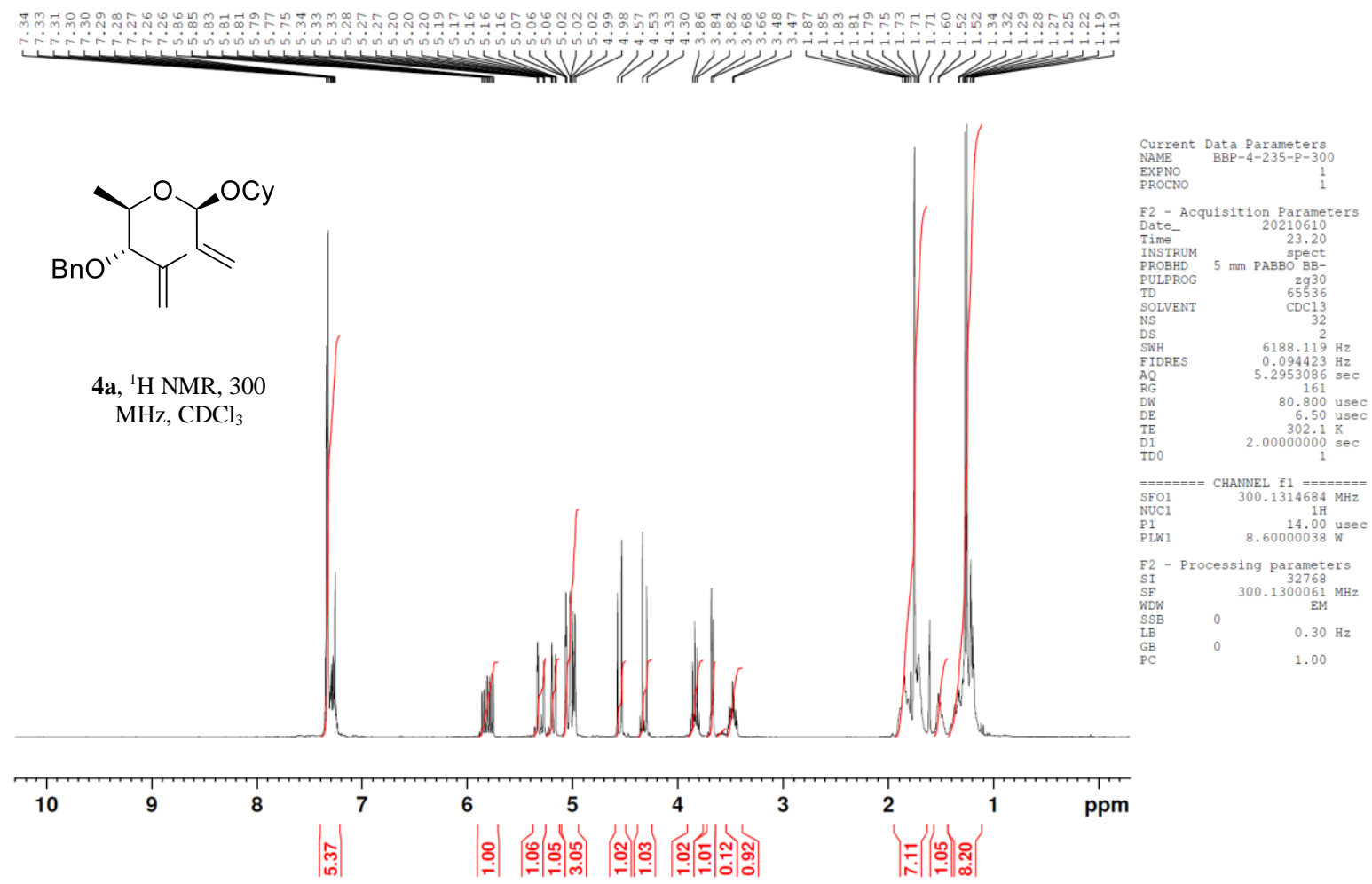

BBP-1-277-P 13120170426
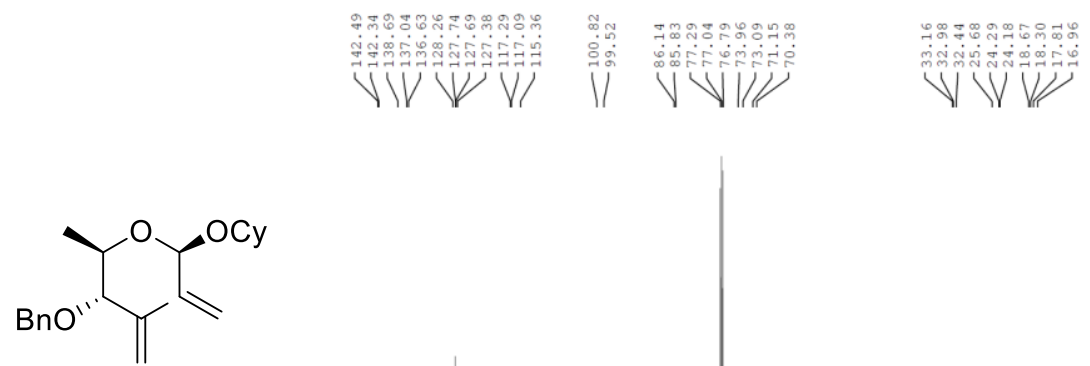

4a, ${ }^{13} \mathrm{C}$ NMR, $125 \mathrm{MHz}$, $\mathrm{CDCl}_{3}$
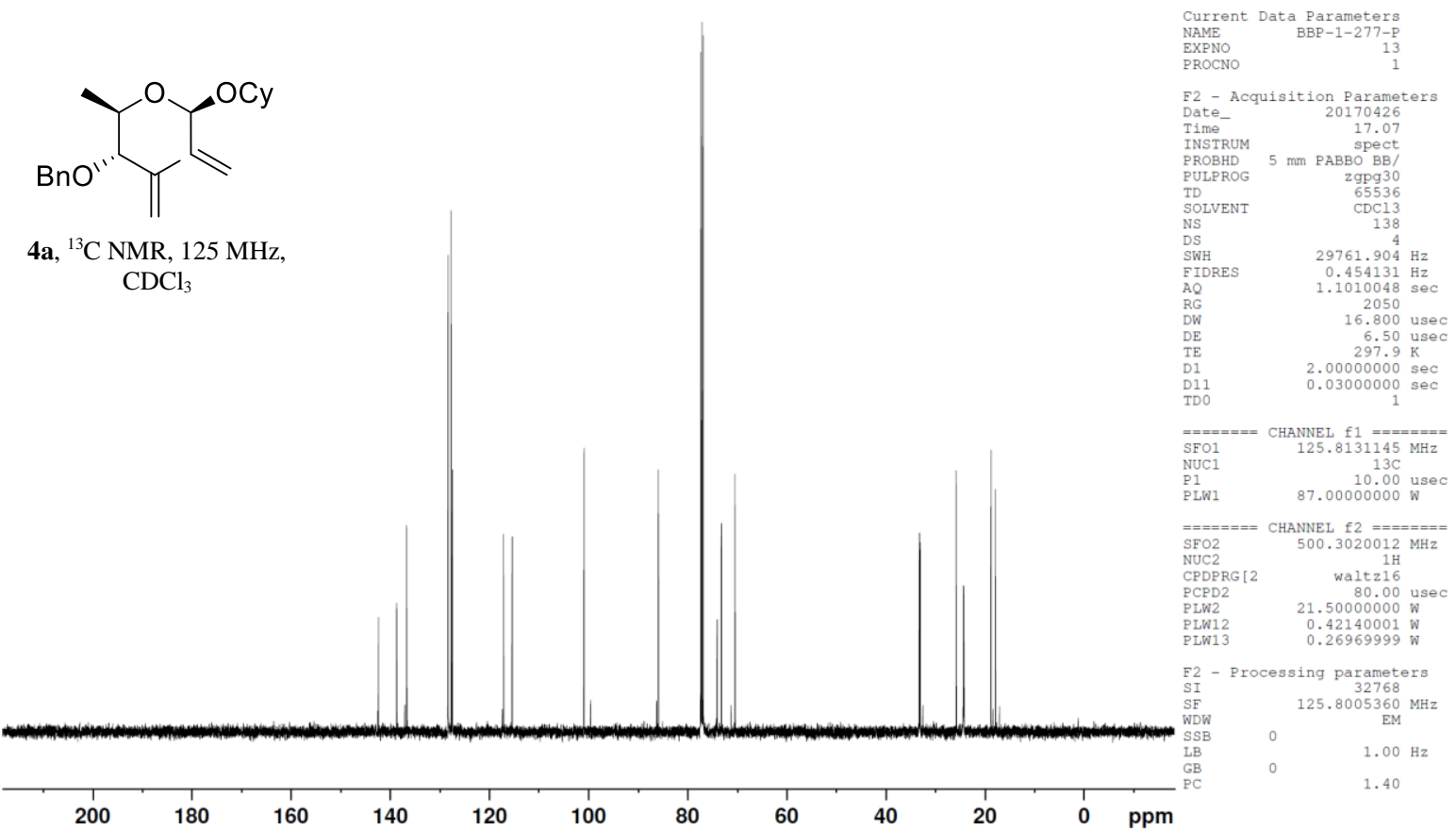
<smiles>CC1=C[C@@H](O[GeH3])O[C@H](C)[C@@H]1Cc1ccccc1</smiles>

7a, ${ }^{1} \mathrm{H}$ NMR, $500 \mathrm{MHz}$ $\mathrm{CDCl}_{3}$

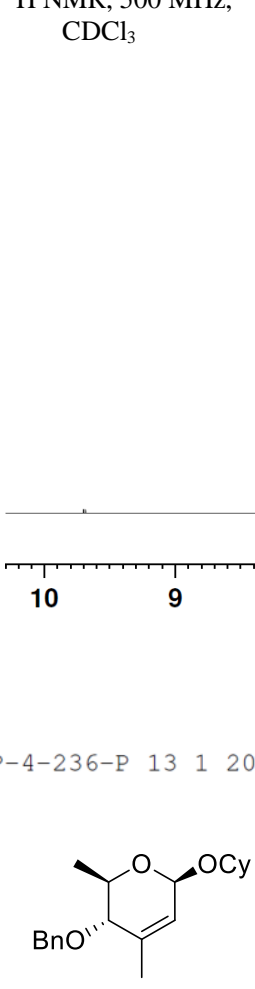

7a, ${ }^{13} \mathrm{C}$ NMR, 125 $\mathrm{MHz}, \mathrm{CDCl}_{3}$
6

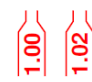

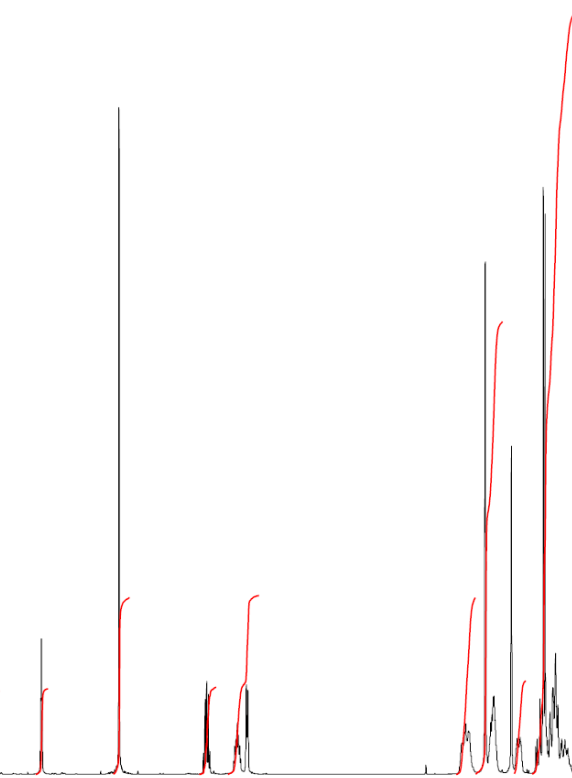

Current Data Parameters
NAME EXPNO

F2 - Acquisition Parameters 20210613
Date INPECT
PROBHD

$\begin{array}{lr}\text { PROBHD } & 5 \mathrm{~mm} \text { PABBO BB/ } \\ \text { PULPROG } & z 930 \\ \text { TD } & 65536 \\ \text { SOLVENT } & \text { CDC13 } \\ \text { TS } & 32\end{array}$ DS SWH $\quad 10000.000 \mathrm{~Hz}$ $\begin{array}{ll}\text { AQ } & 3.152588 \mathrm{~Hz} \\ \mathrm{RG} & 3.2767999 \mathrm{sec}\end{array}$ $\begin{array}{lr}\mathrm{DW} & 50.000 \text { use } \\ \mathrm{DE} & 6.50 \text { usec }\end{array}$ TE $\quad 2.50000000 \mathrm{Kec}$ $=======$ CHANNEL $\mathrm{f} 1 \mathrm{l}=======$
$500.3030896 \mathrm{MHz}$
SFO1 11.50 use $\begin{array}{lcc}\text { F2 } & \text { Processing parameters } \\ \text { SI } & 65536 \\ \text { SE } & 500.3000134 \mathrm{MHz} \\ \text { WDW } & 0 & \text { EM } \\ \text { SSB } & 0 & 0.30 \mathrm{~Hz} \\ \text { LB } & 0 & \\ \text { GB } & 0 & \end{array}$ BBP-4-236-P 131202106010

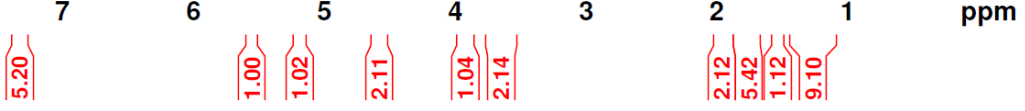

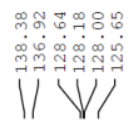 \\ $\mid V^{\infty}$ \\ (n)}


MJK-5-039-P 11 yhr 20170207

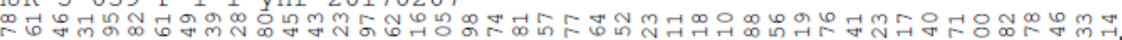

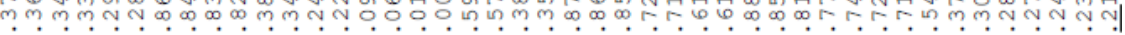

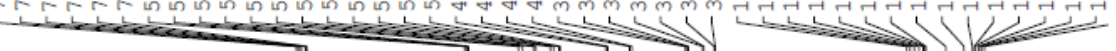<smiles>C=C1C(=C)[C@H](O[GaH])[C@@H](C)O[C@H]1Cc1ccccc1</smiles>

4b, ${ }^{1} \mathrm{H}$ NMR, $500 \mathrm{MHz}$, $\mathrm{CDCl}_{3}$
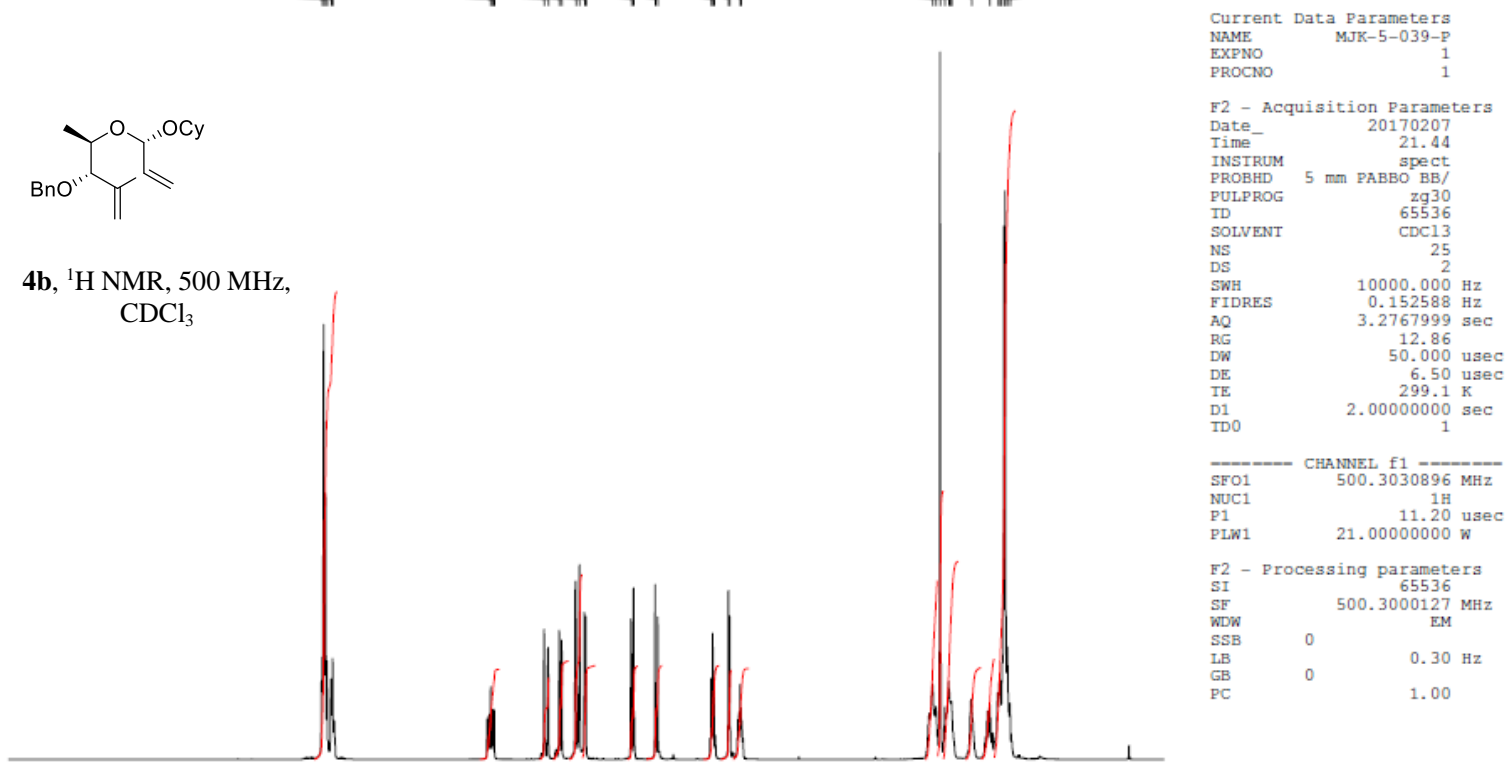

$10 \quad 9$

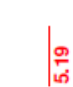

|

| (8)

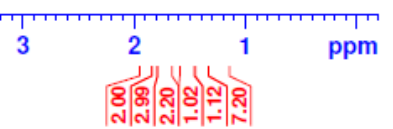

MJK-5-039-P 11 yhr 20170207
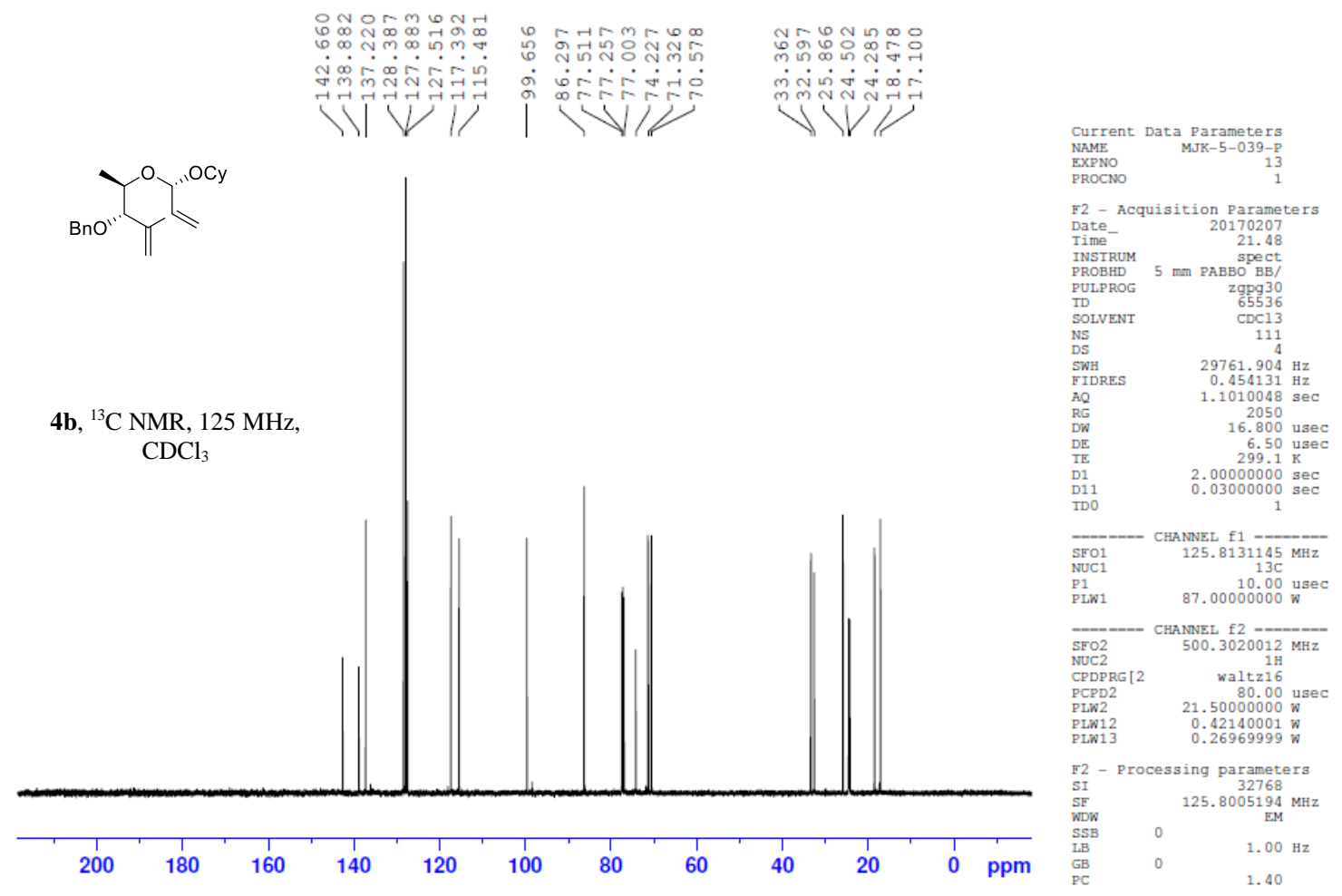


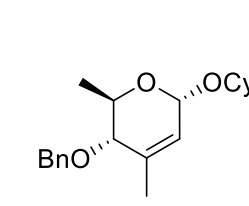

7b, ${ }^{1} \mathrm{H}$ NMR, $500 \mathrm{MHz}$, $\mathrm{CDCl}_{3}$
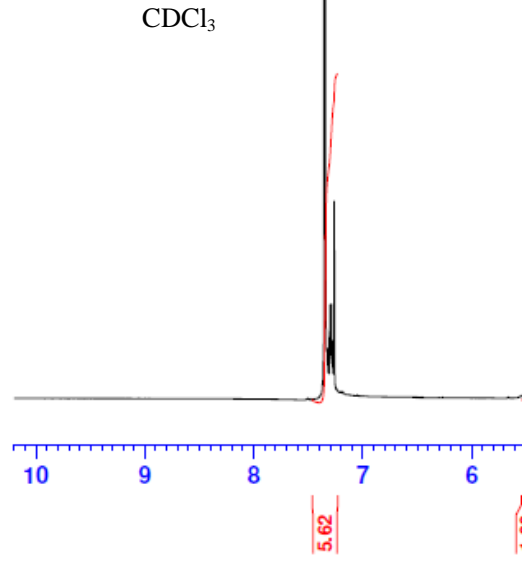

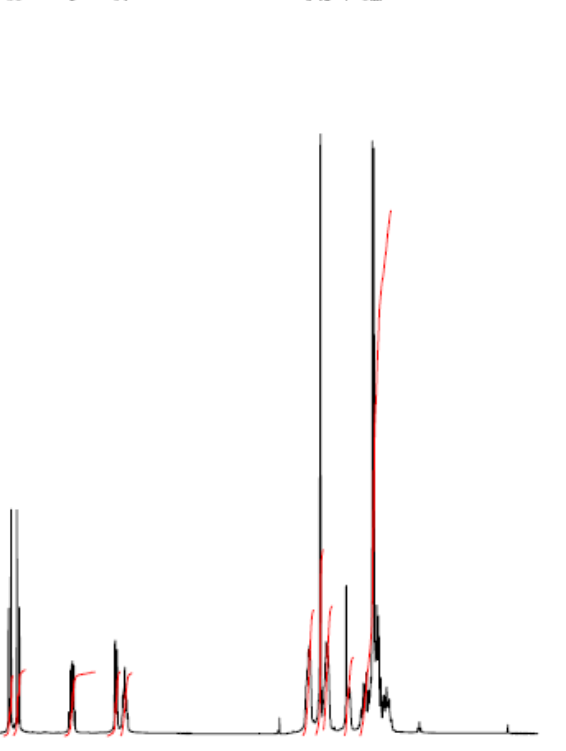

$1+1+101110$

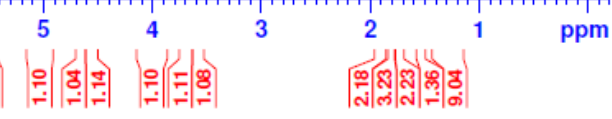

MJK-5-040-P 131 yhr 20170208

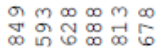

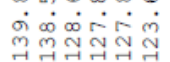<smiles>CC1=C[C@@H](O[GaH2])[C@H]([18OH])[C@H](C)O1</smiles>

7b, ${ }^{13} \mathrm{C}$ NMR, 125 $\mathrm{MHz}, \mathrm{CDCl}_{3}$

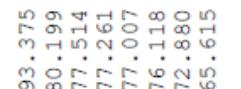

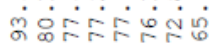

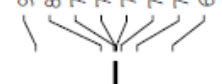

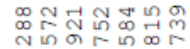

我

UVV
Current Data Parameters

EXPN

F2 - Acquisition Parameters

20170208
Date-
Time-

spect
INSTRUM
PROBHD
mUm PABBO BB/

$\begin{array}{rr}\text { PULPROG } & \text { zg30 } \\ \text { TD } & 65536\end{array}$

SOLVENT

NS

SWH $\quad 10000.000 \mathrm{~Hz}$

$\begin{array}{lr}0.152588 \mathrm{~Hz} \\ \text { AQ } & 3.2767999 \mathrm{sec} \\ 99.44 & \end{array}$

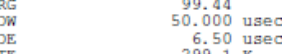

$\begin{array}{ll}\text { TE } & 2.00000000 \mathrm{sec} \\ \text { D1 } & 1\end{array}$

SFO1 CHANNEL f1 - $500.3030896 \mathrm{MHz}$

$\begin{array}{lr}\text { SFO1 } & 1 \mathrm{H} \\ \text { N11 } & 11.20 \mathrm{usec} \\ \text { PLW1 } & 21.00000000 \mathrm{w}\end{array}$

F2 - Processing parameters

$\begin{array}{lc}\text { SI } & 65536 \\ \text { SF } & 500.3000135 \\ \text { WDW } & \text { EM }\end{array}$

$\begin{array}{lll}\text { SSB } & 0 & 0.30 \mathrm{~Hz} \\ \mathrm{LB} & 0 & \\ \mathrm{~GB} & \end{array}$
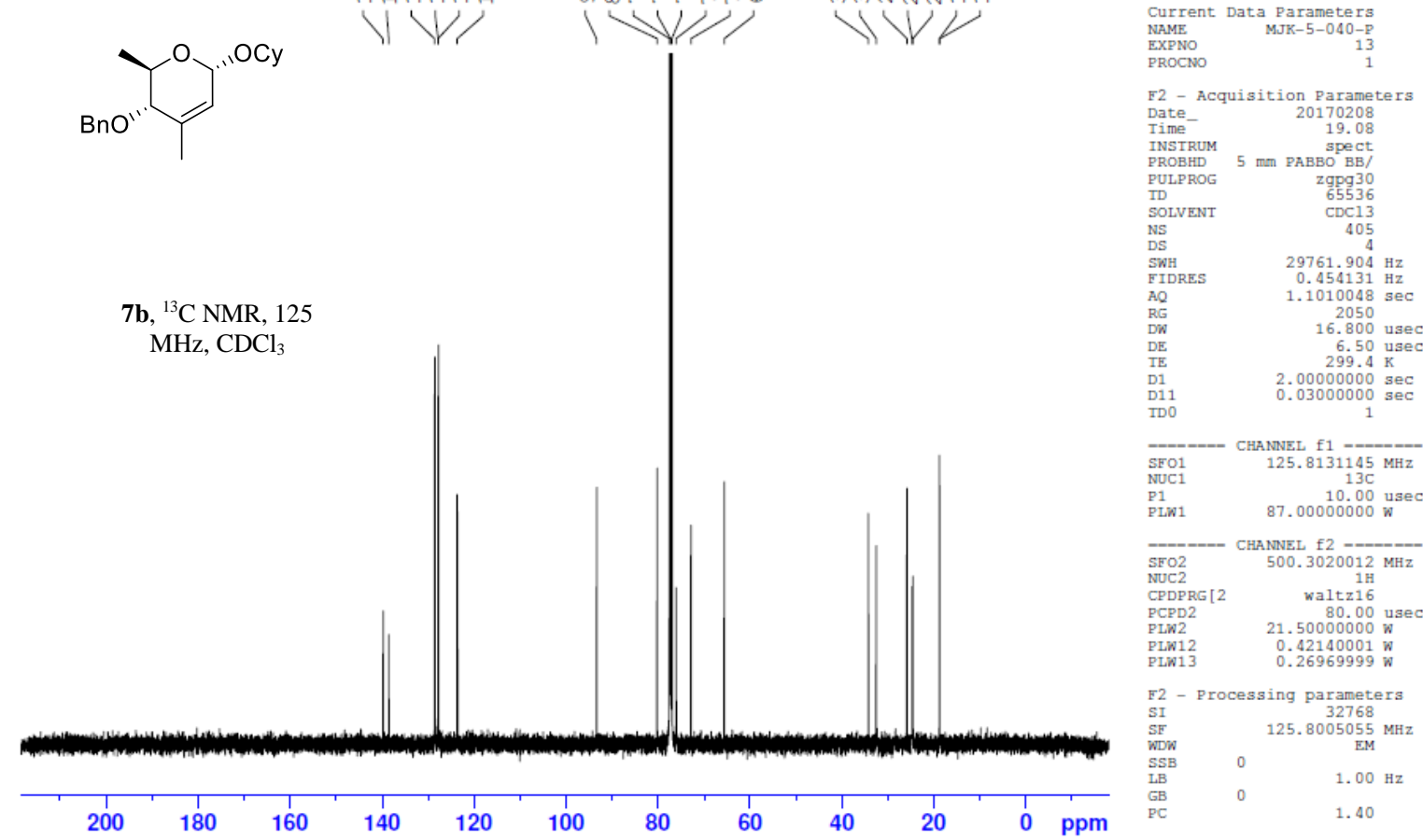


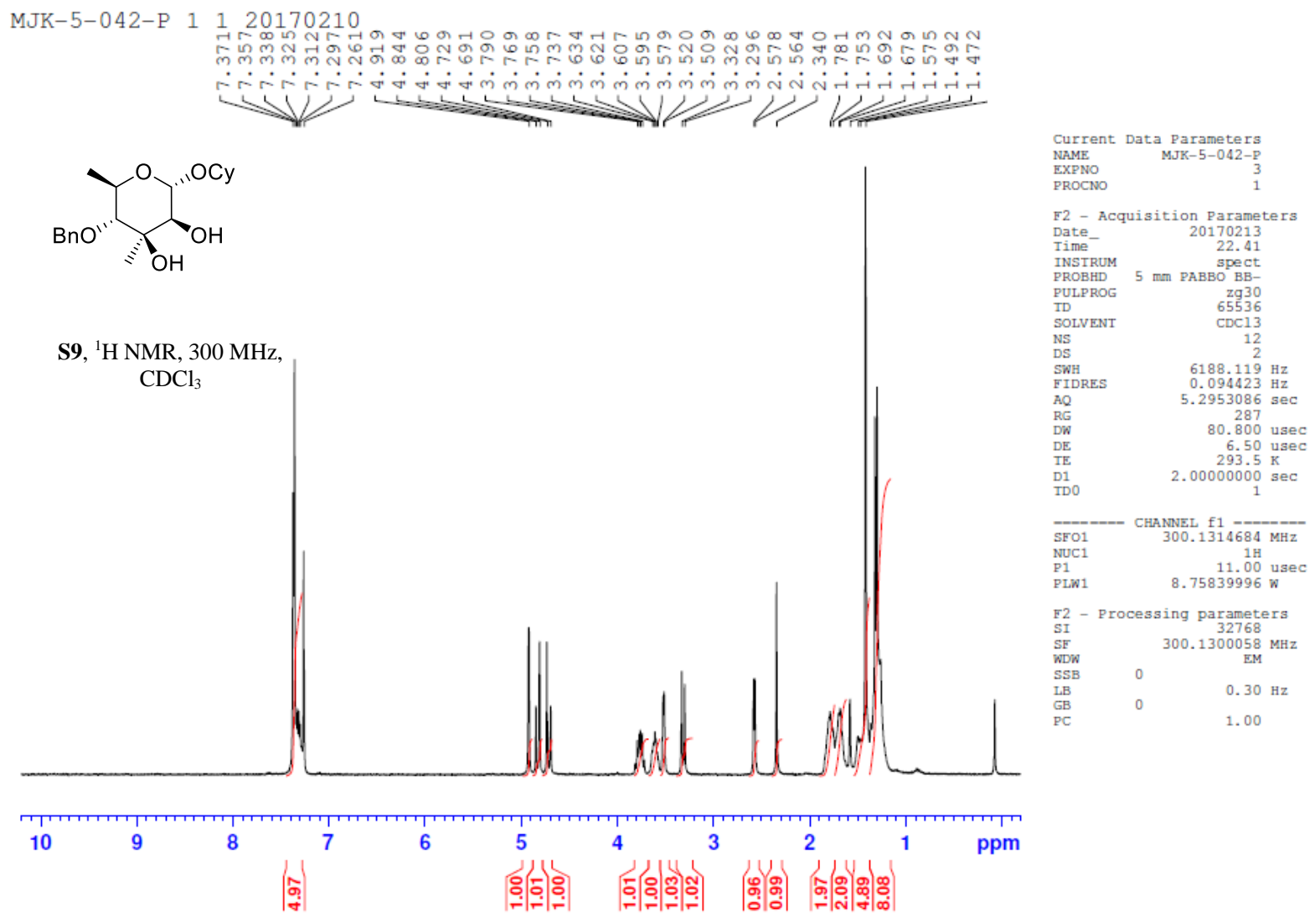

MJK-5-042-P 13120170210
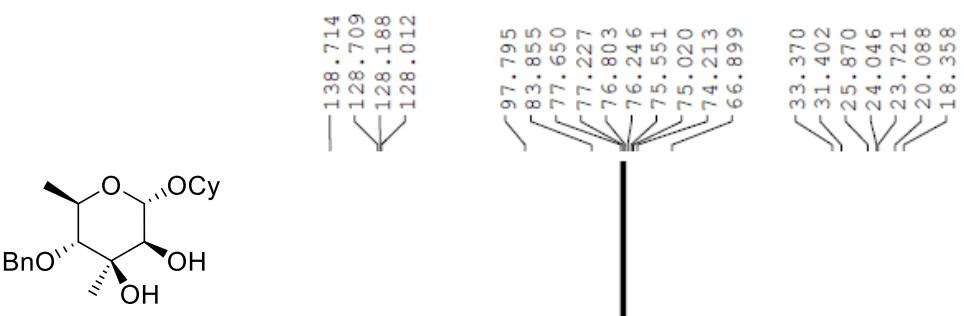

S9, ${ }^{13} \mathrm{C} \mathrm{NMR}, 75 \mathrm{MHz}$, $\mathrm{CDCl}_{3}$
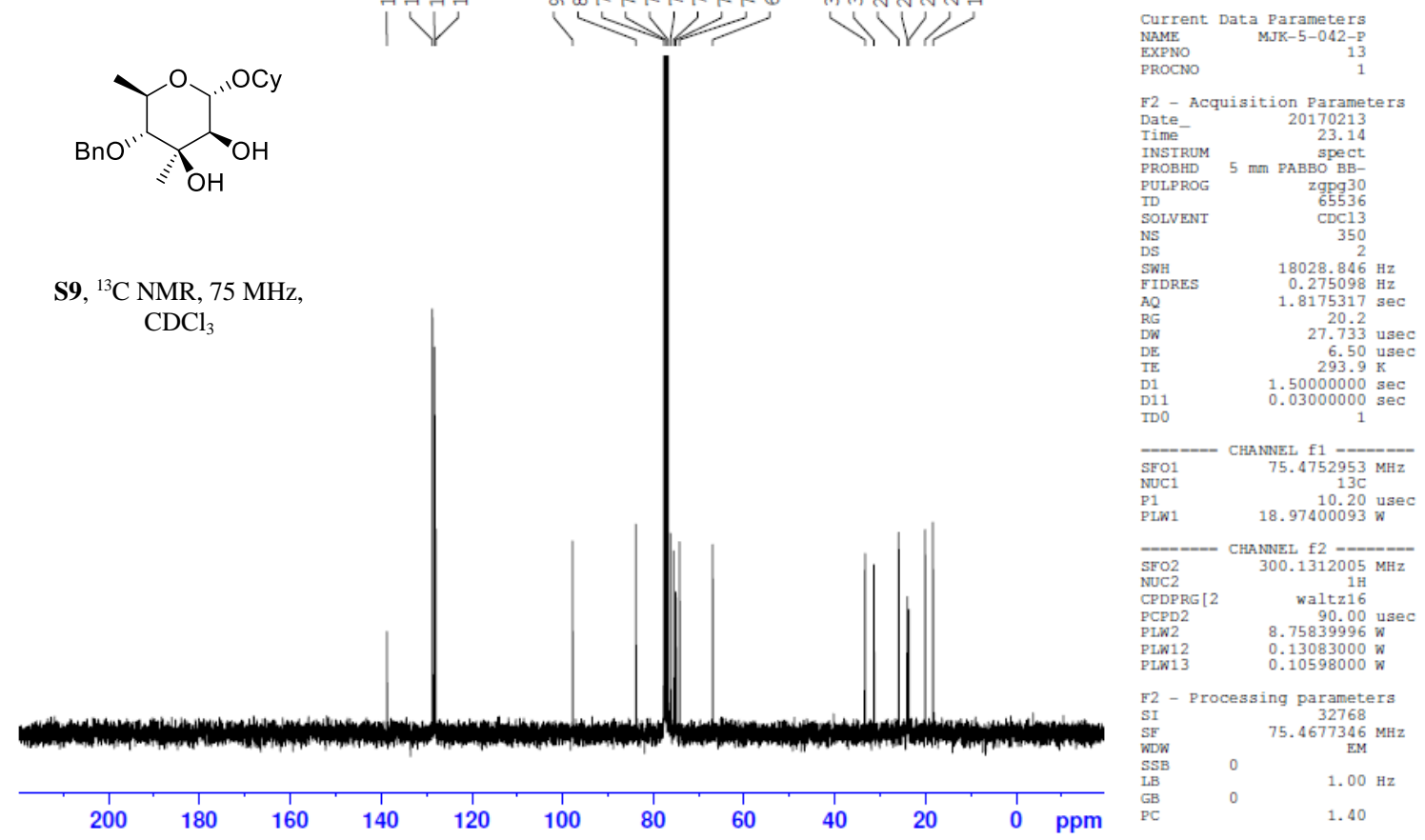


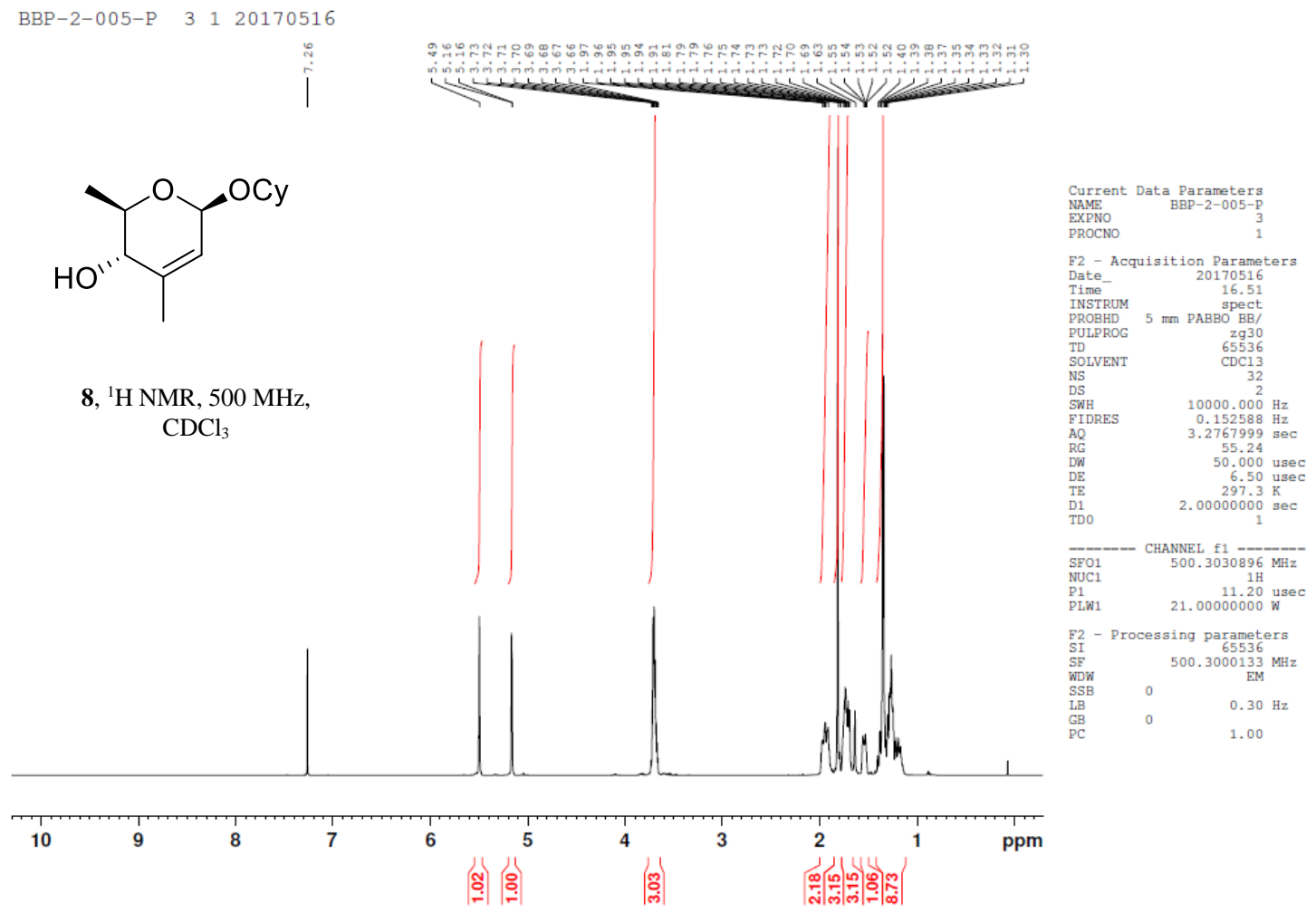

BBP-2-005-P 133120170516
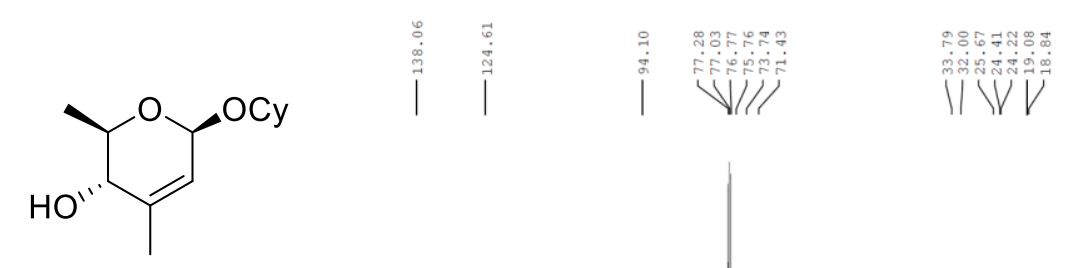

8, ${ }^{13} \mathrm{C}$ NMR, $125 \mathrm{MHz}$,

$\mathrm{CDCl}_{3}$
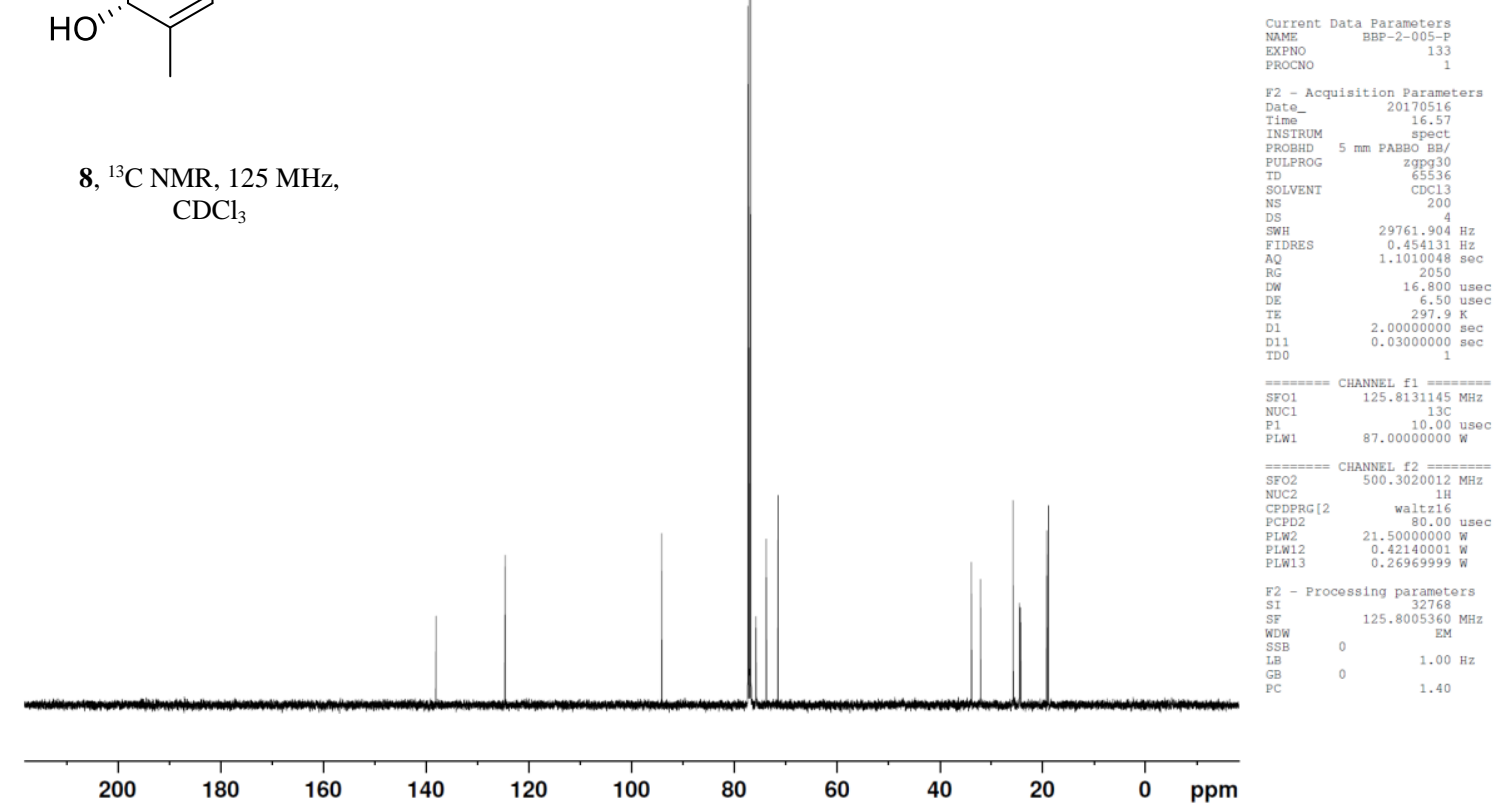


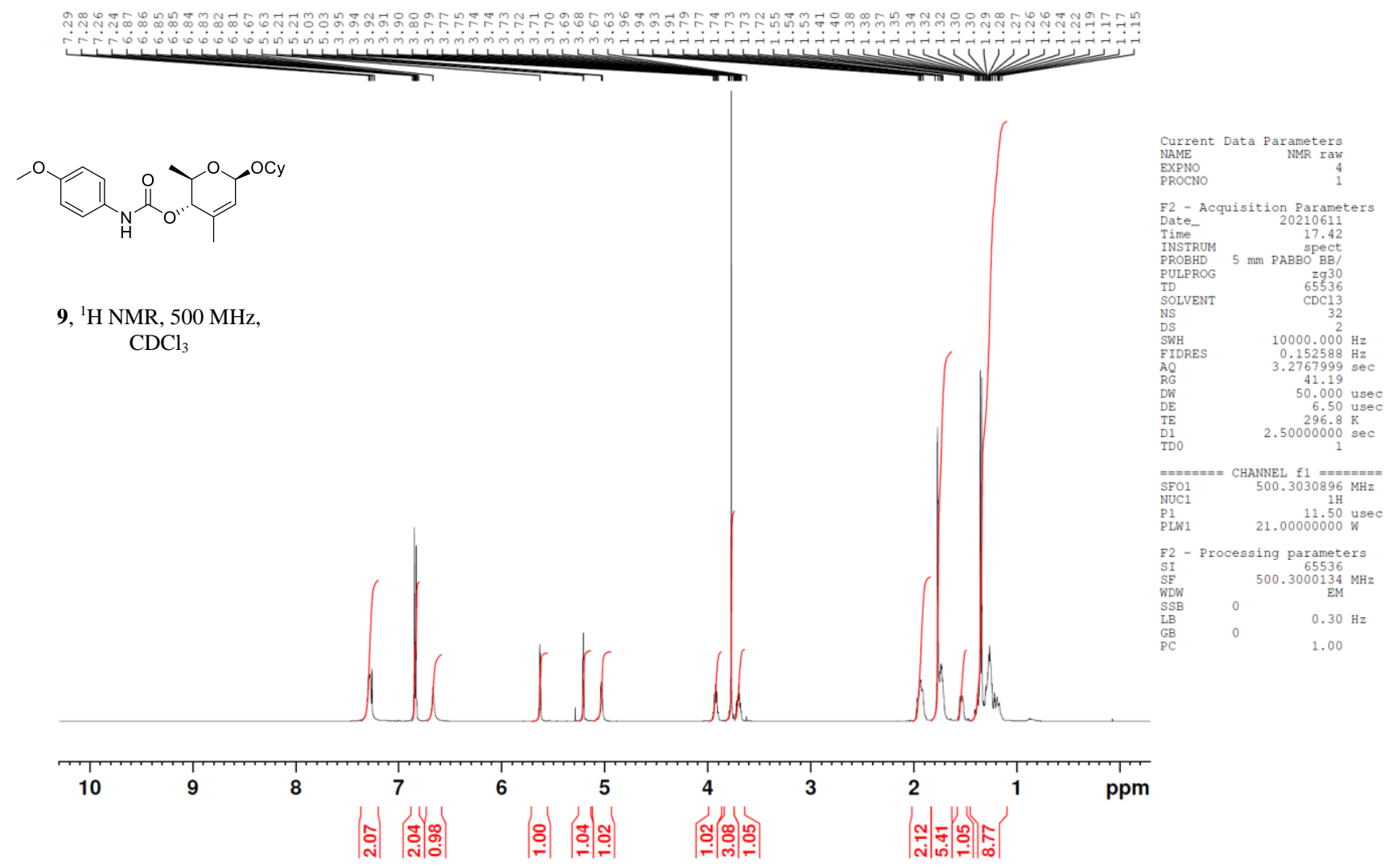

BBP-4-148-P 1361202106010
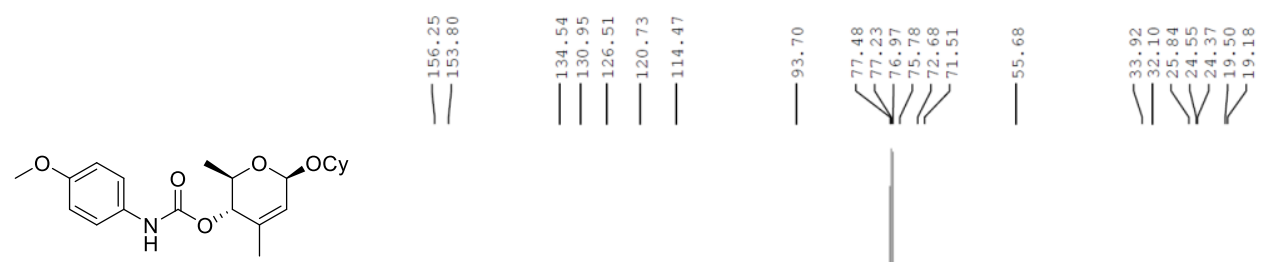

9, ${ }^{13} \mathrm{C}$ NMR, $125 \mathrm{MHz}$, $\mathrm{CDCl}_{3}$
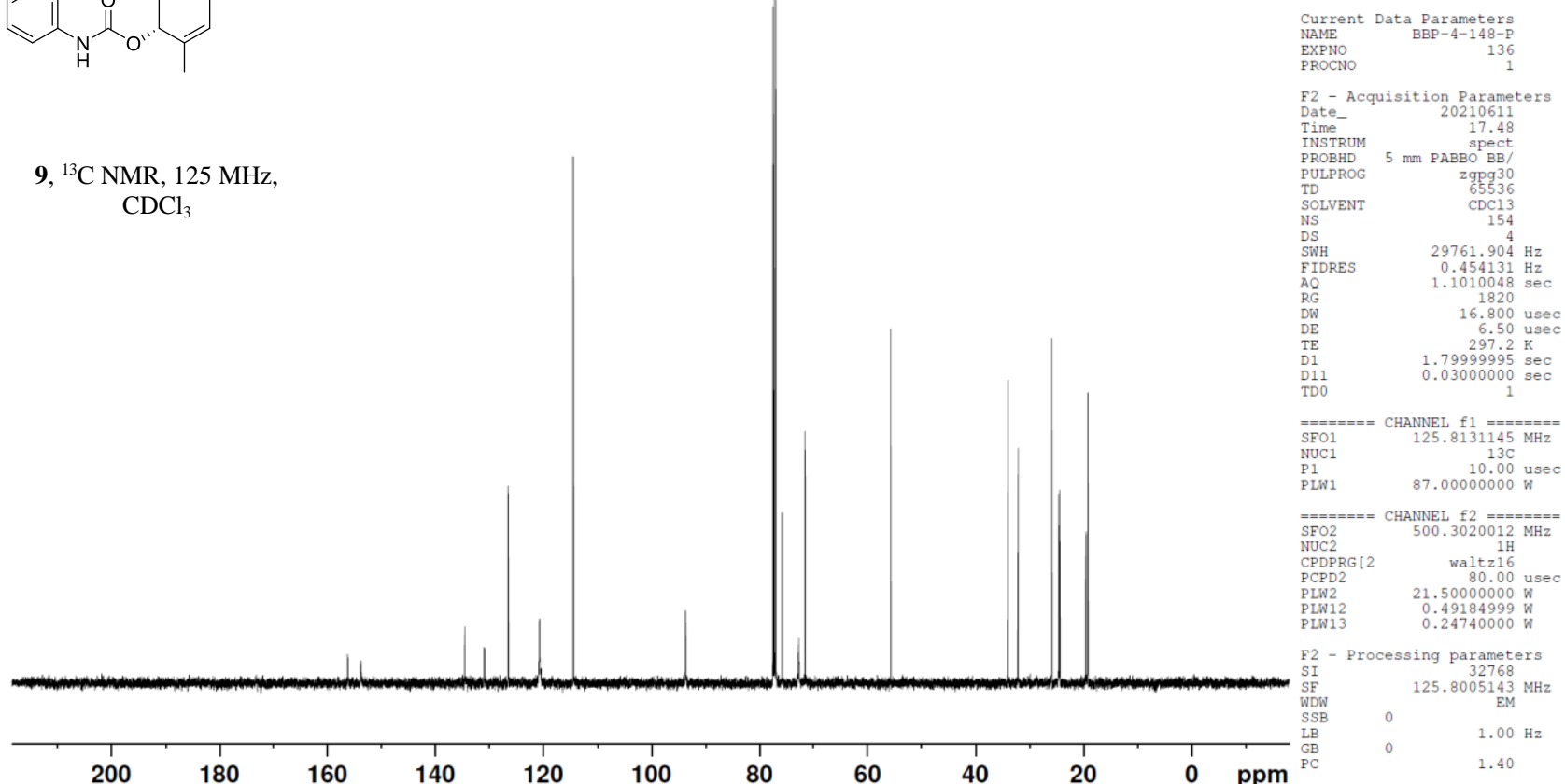

S 


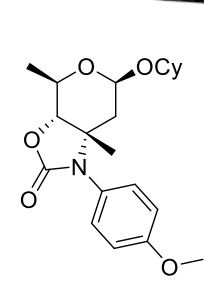

10, ${ }^{1} \mathrm{H}$ NMR, $500 \mathrm{MHz}$, $\mathrm{CDCl}_{3}$
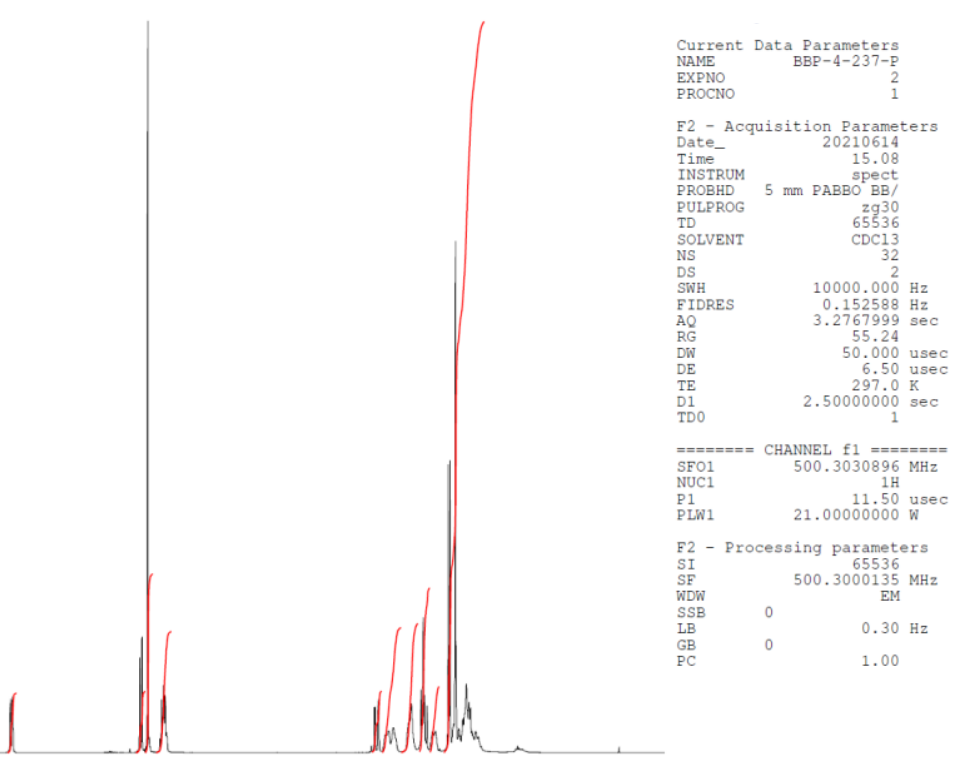

BBP-4-237-P 131202106013
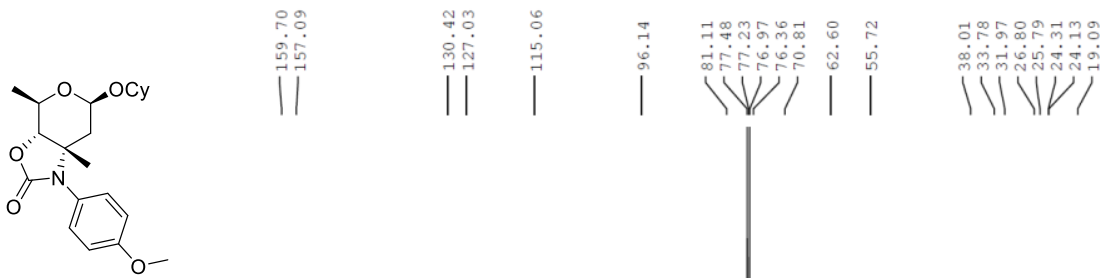

10, ${ }^{13} \mathrm{C}$ NMR, 125

$\mathrm{MHz}, \mathrm{CDCl}_{3}$

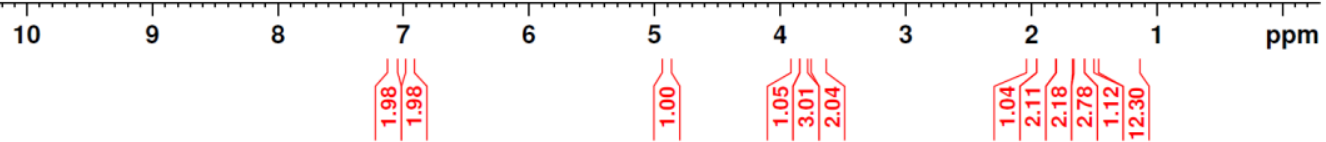

ppm
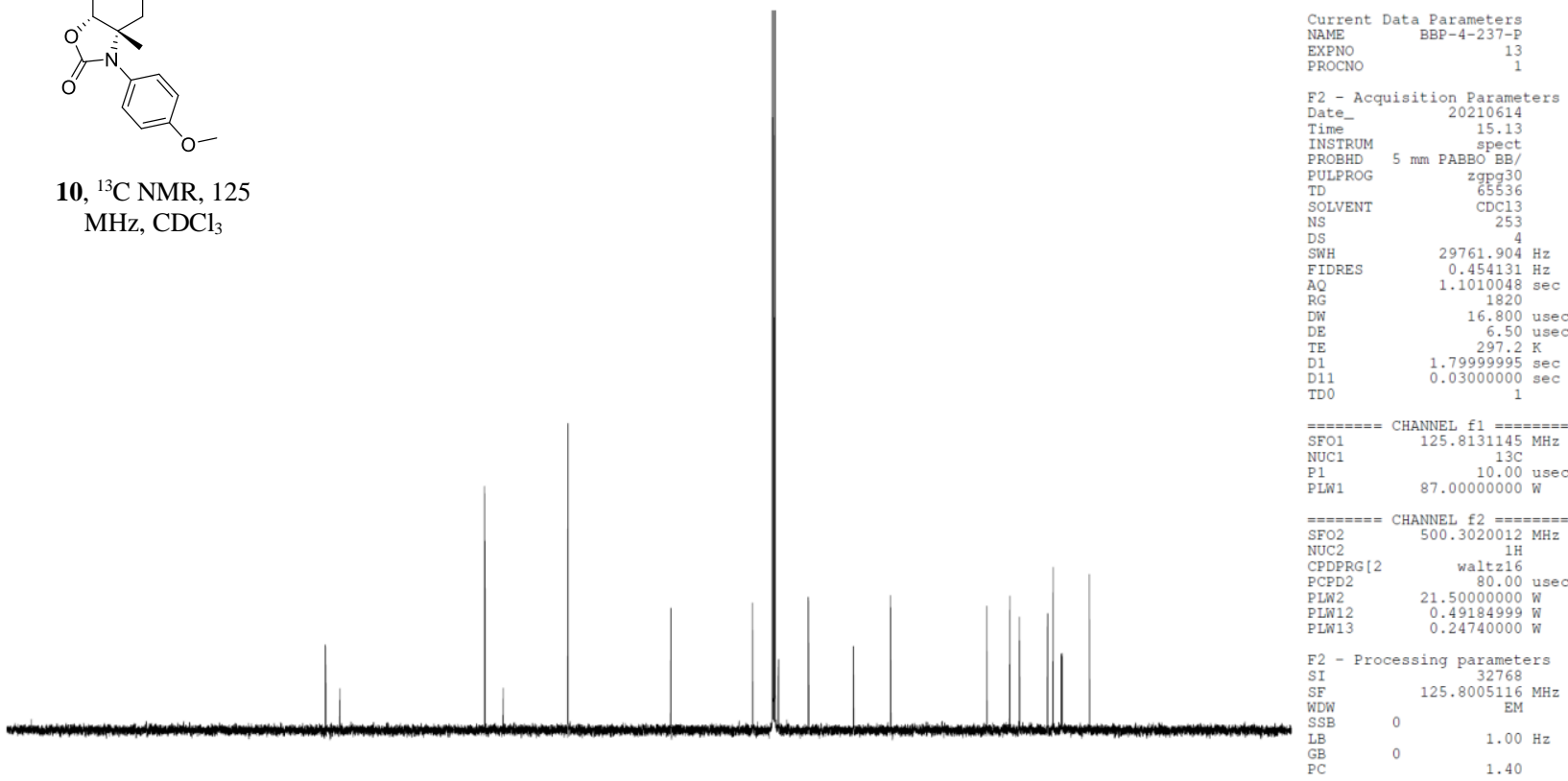

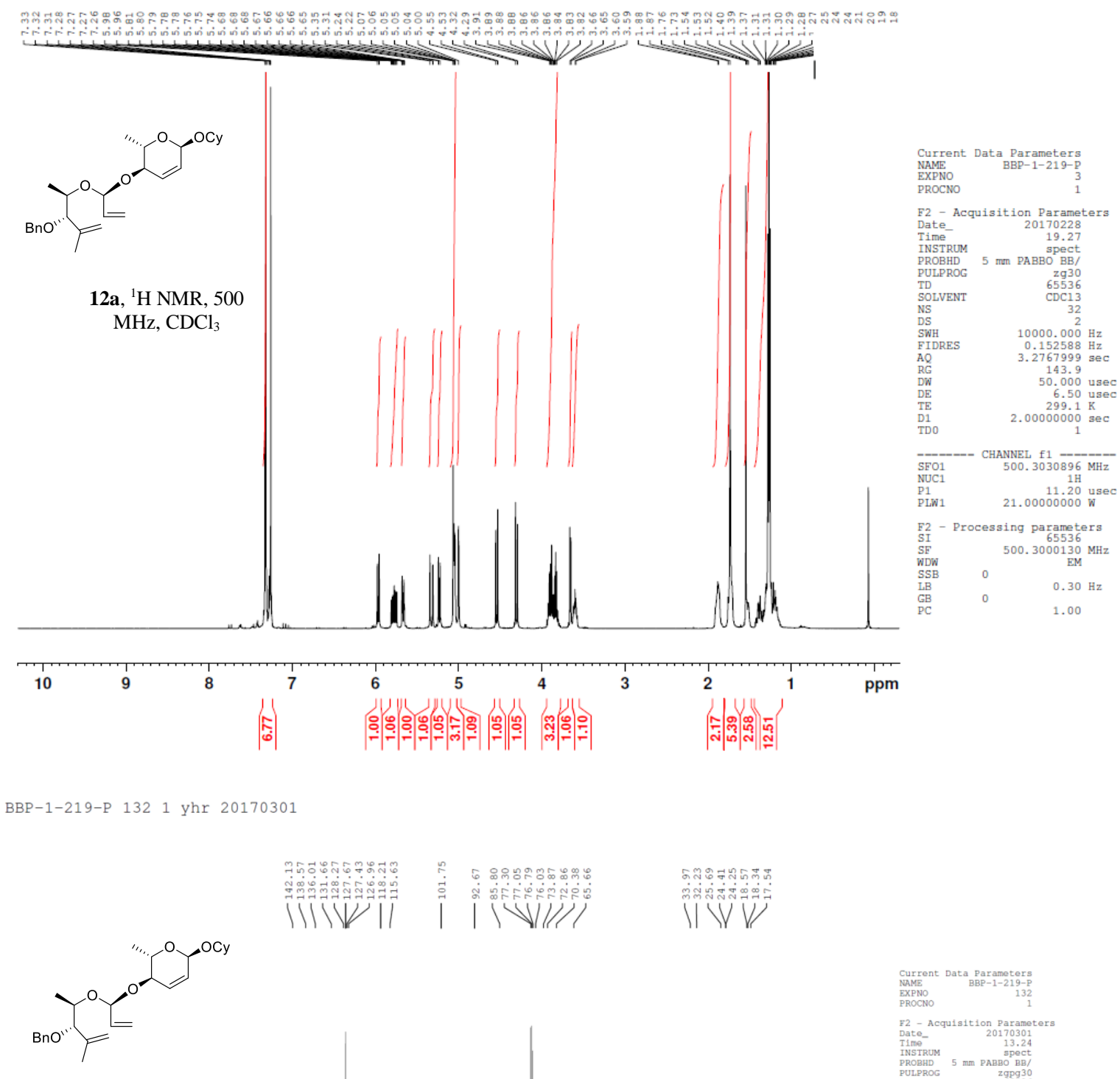

12a, ${ }^{13} \mathrm{C}$ NMR, $125 \mathrm{MHz}$, $\mathrm{CDCl}_{3}$
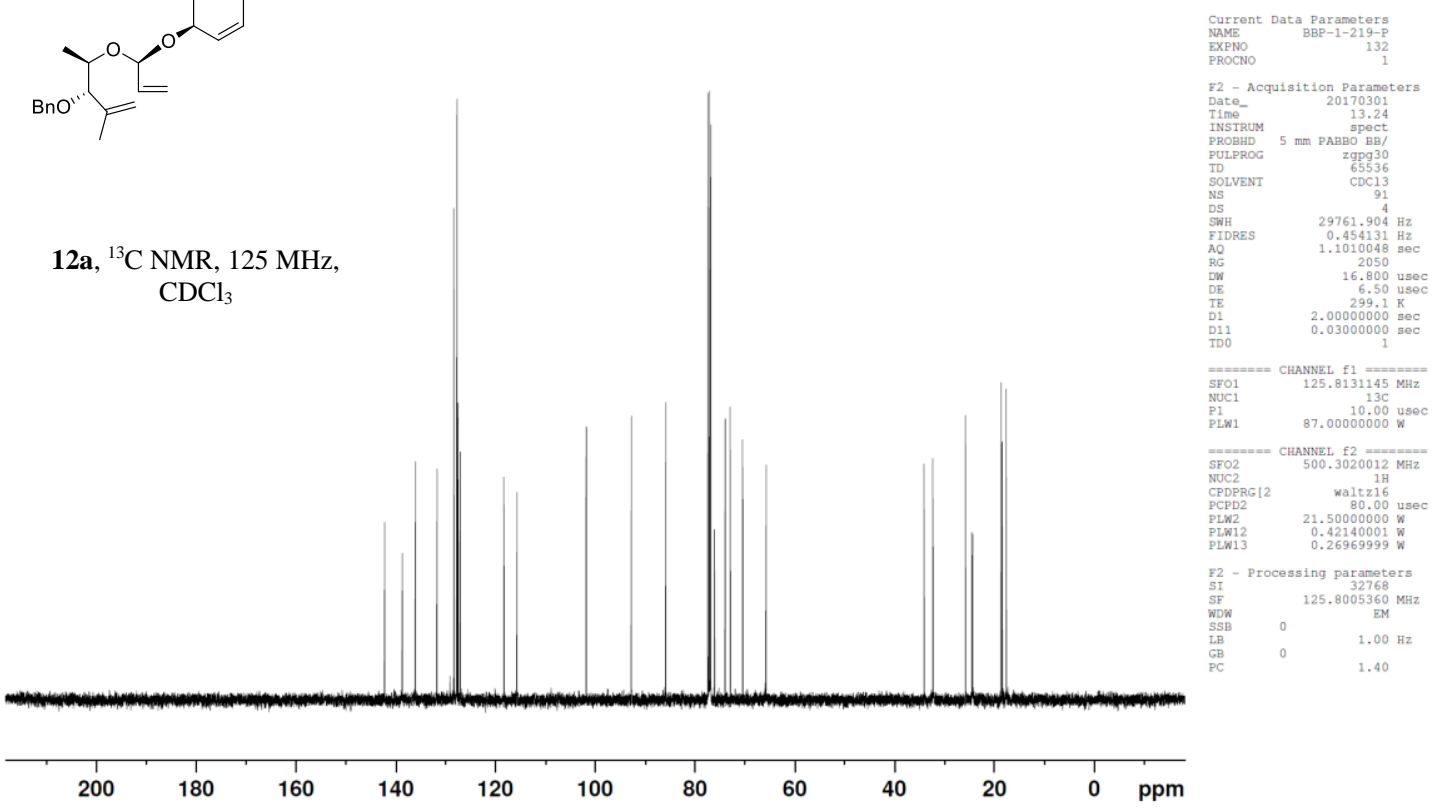


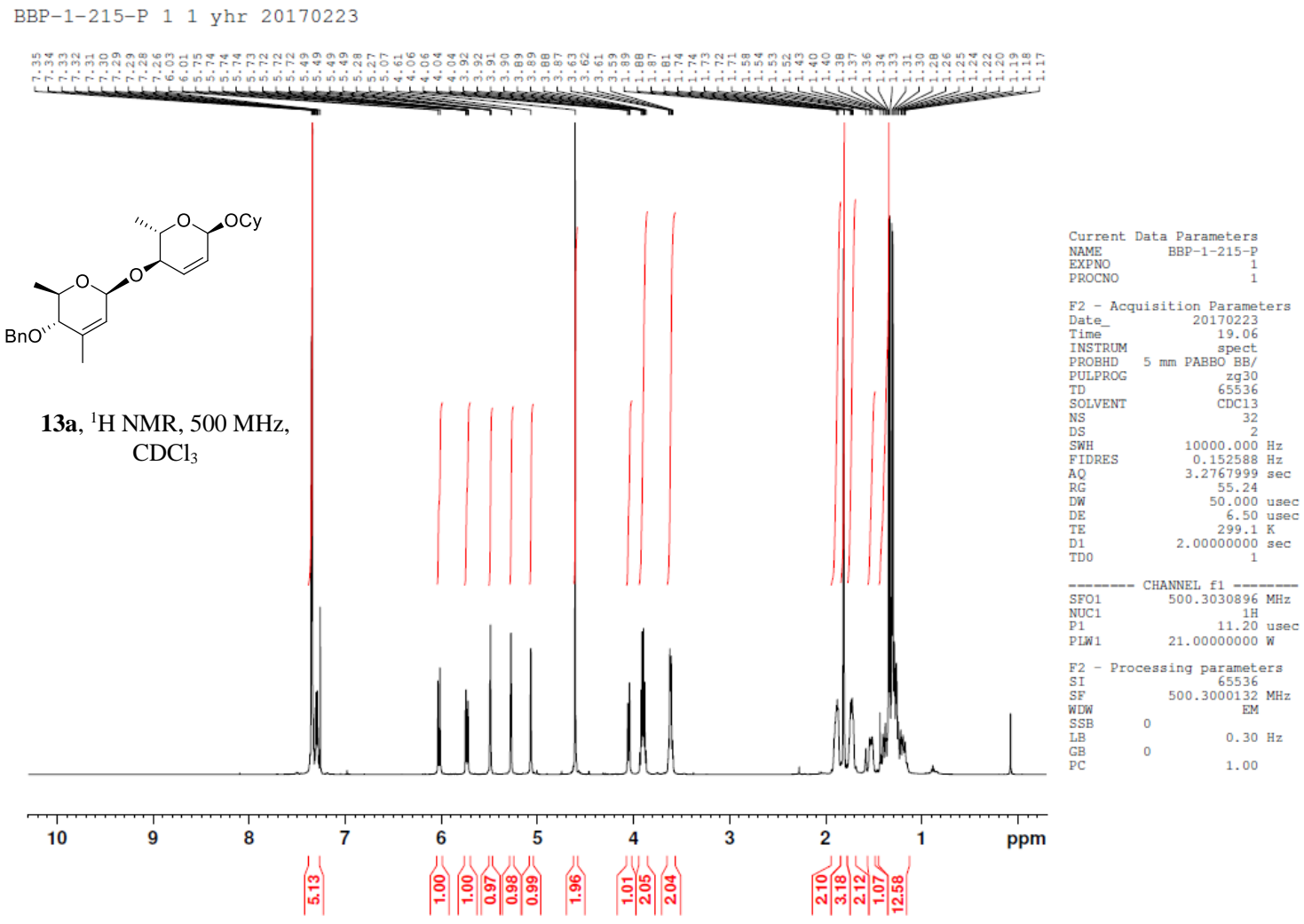

BBP-1-205-P 1321 yhr 20170211
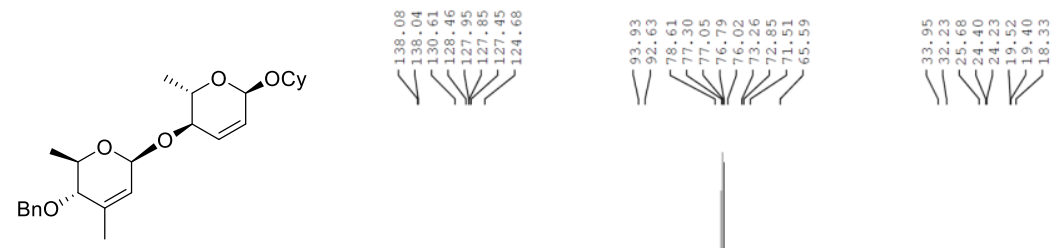

13a, ${ }^{13} \mathrm{C}$ NMR, 125 $\mathrm{MHz}, \mathrm{CDCl}_{3}$

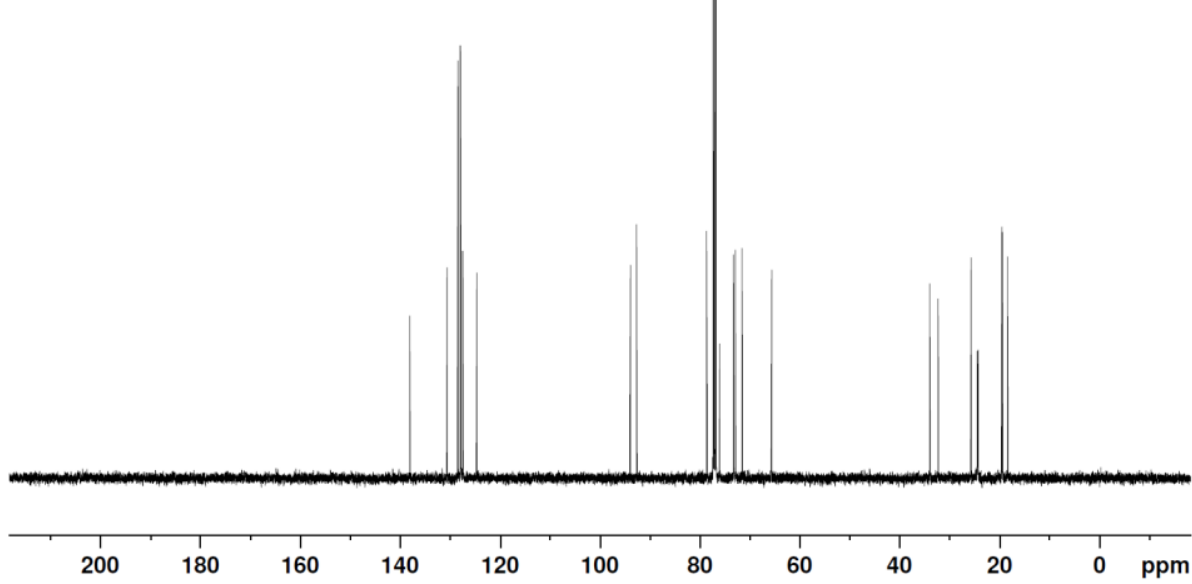




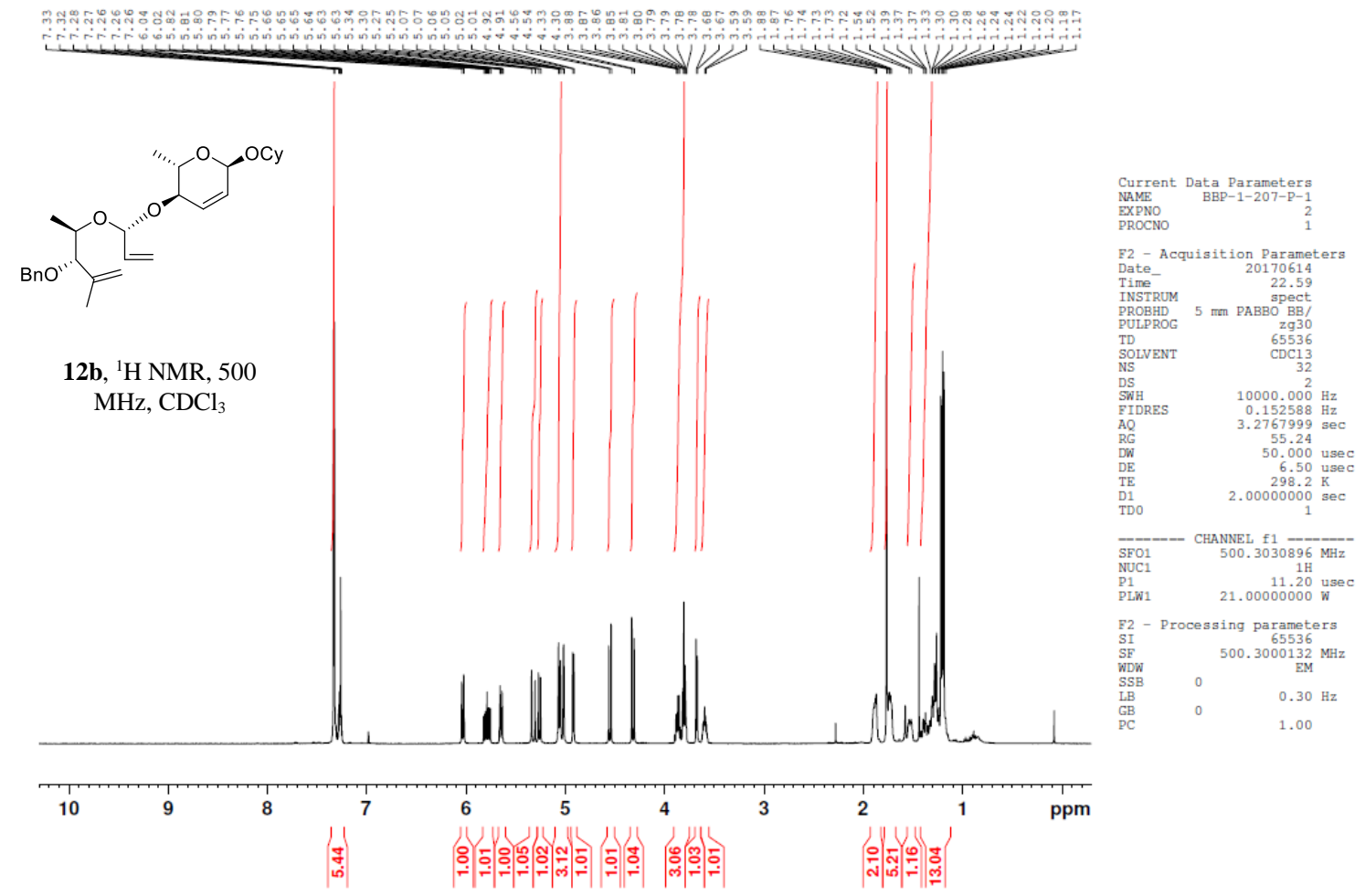

BBP-1-207-P-2 13

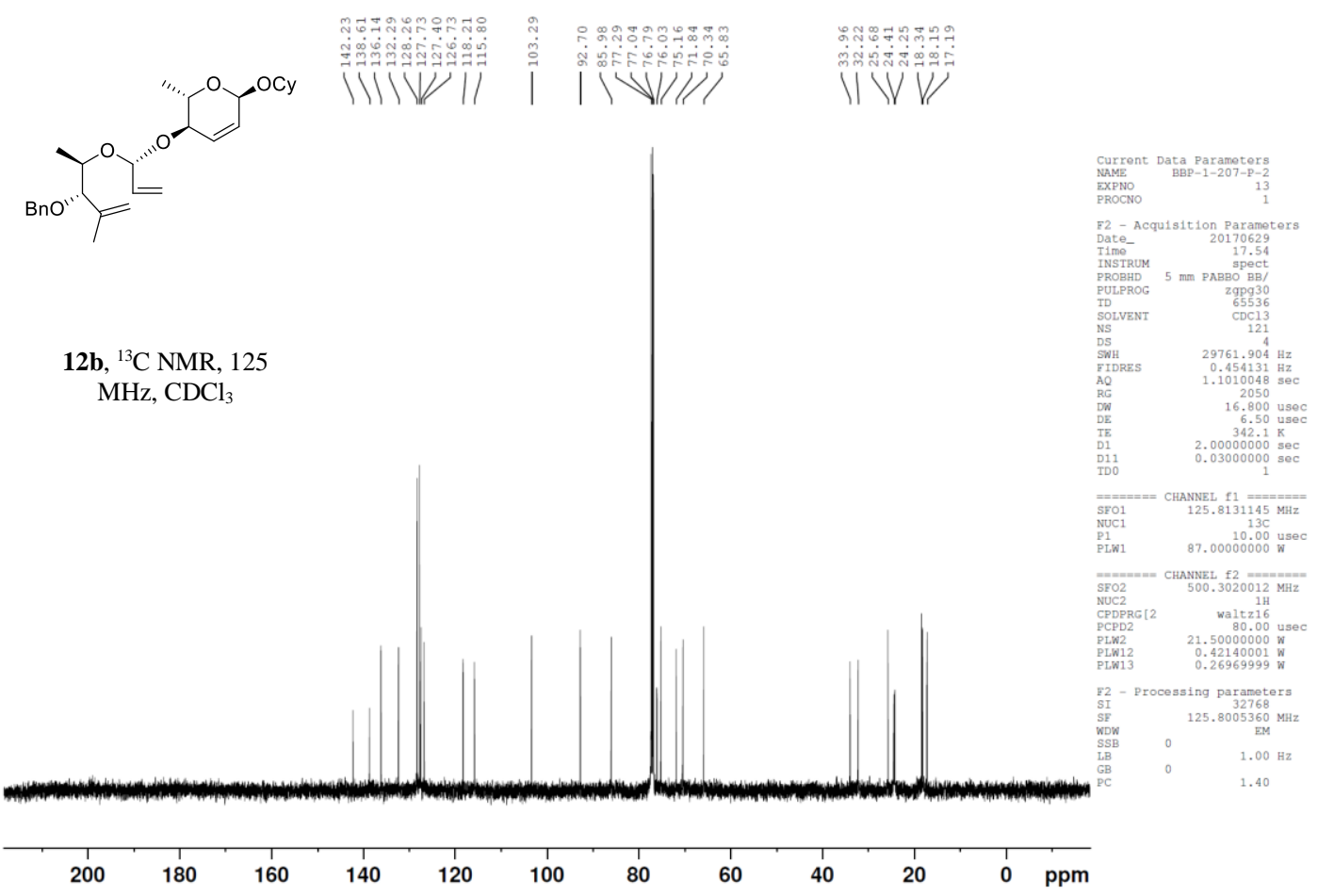



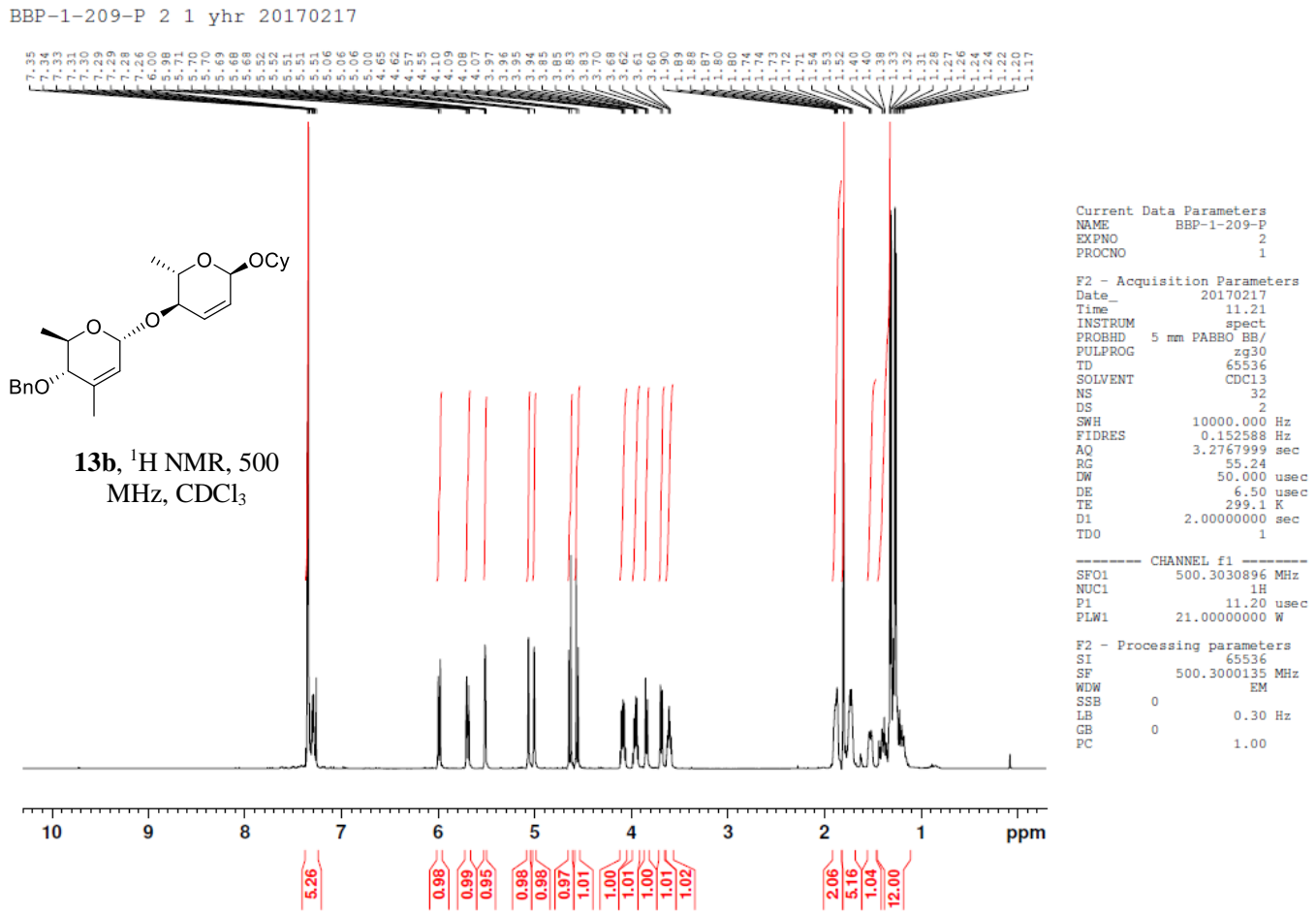

BBP-1-209-P 1321 yhr 20170217
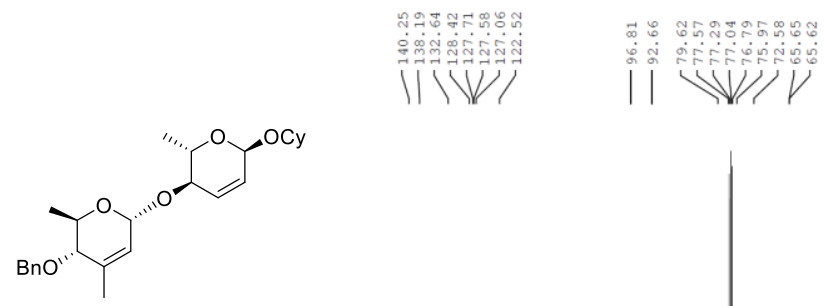

|

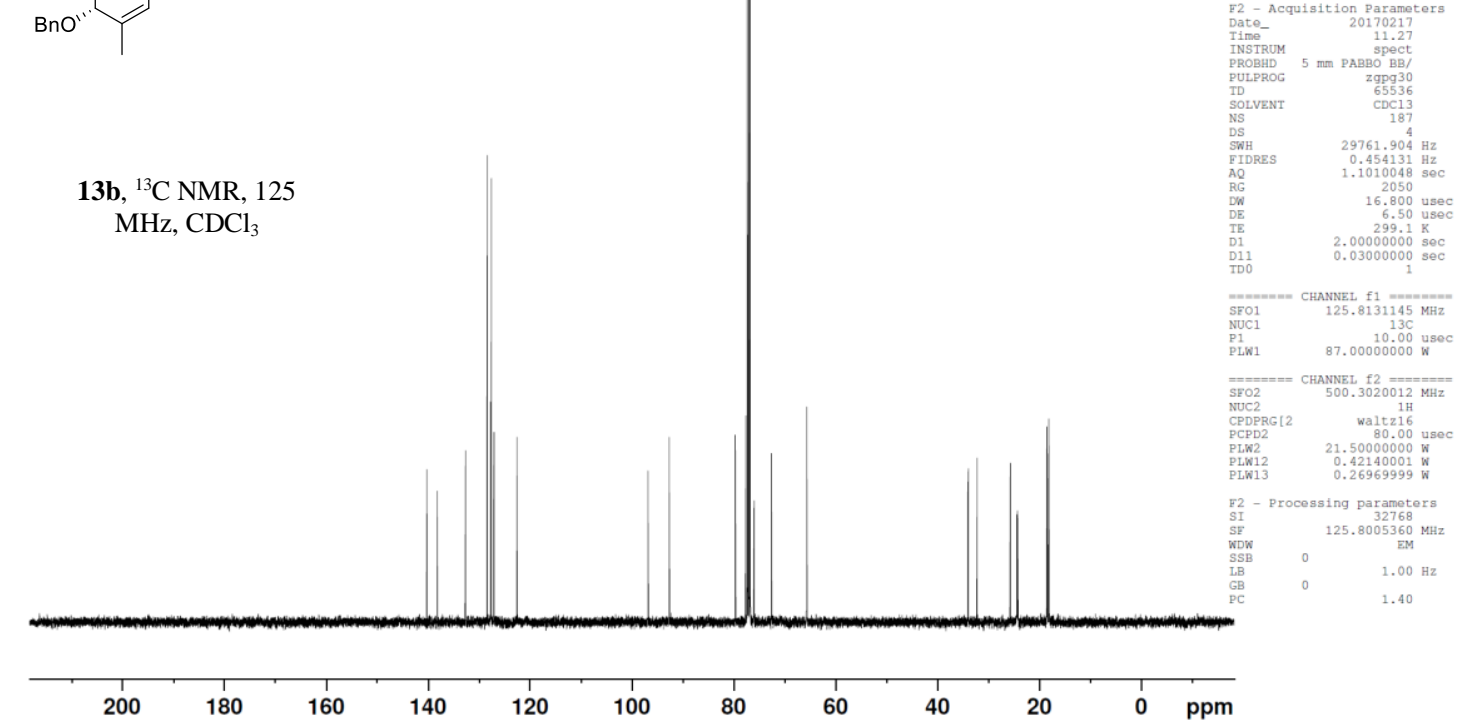


BBP-1-211-P 21 yhr 20170221

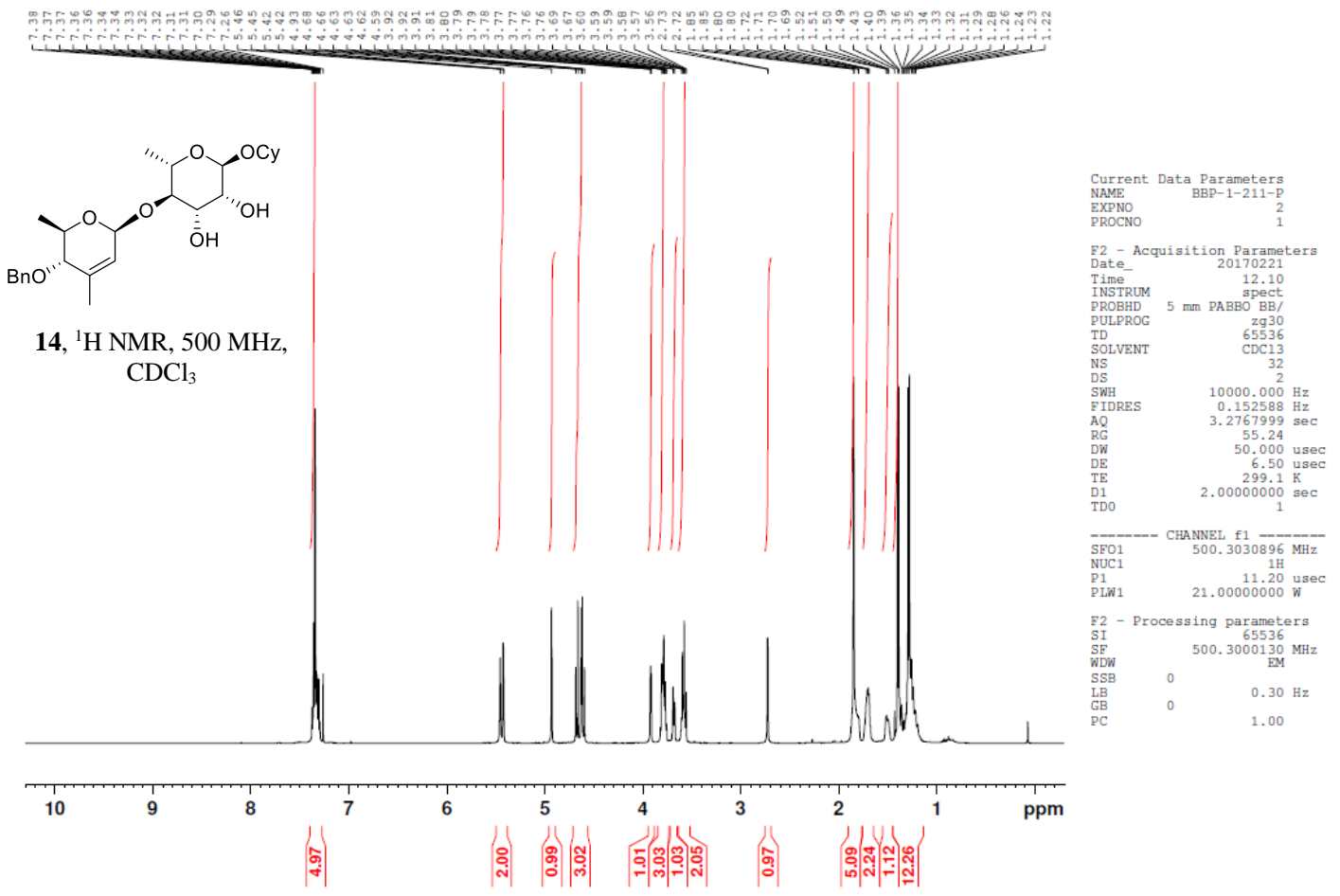

BBP-1-211-P 1321 yhr 20170221
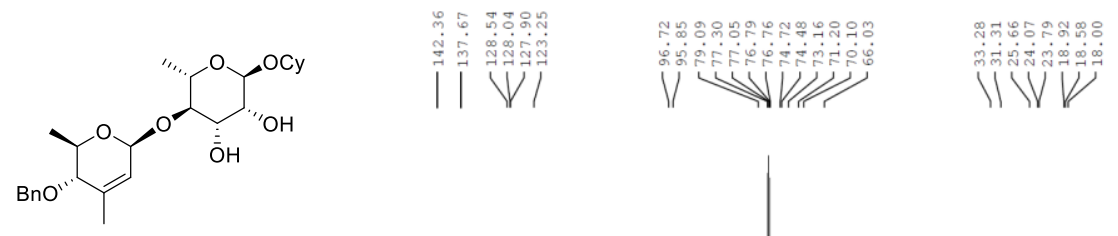

14, ${ }^{13} \mathrm{C}$ NMR, 125

$\mathrm{MHz}, \mathrm{CDCl}_{3}$
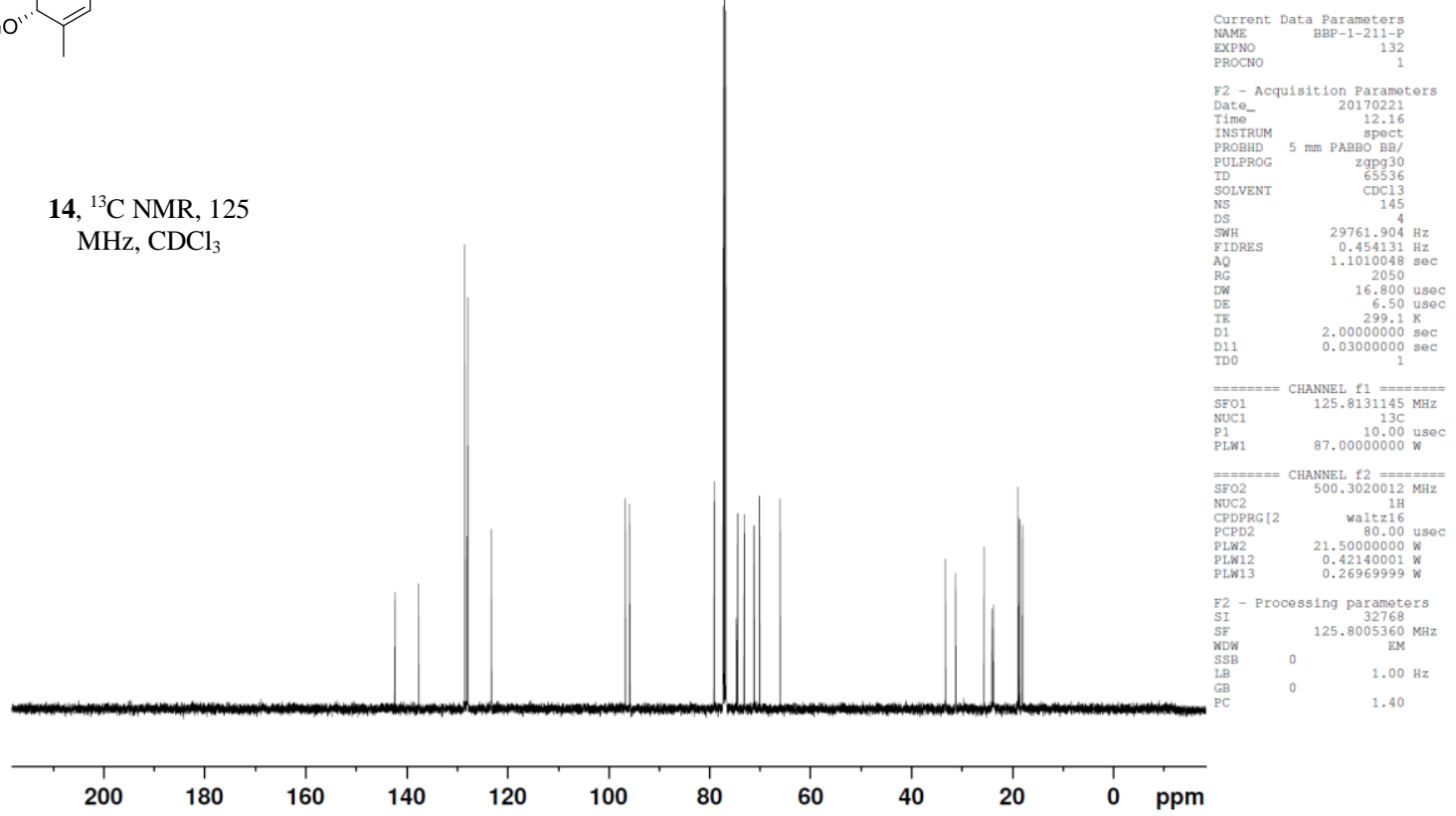


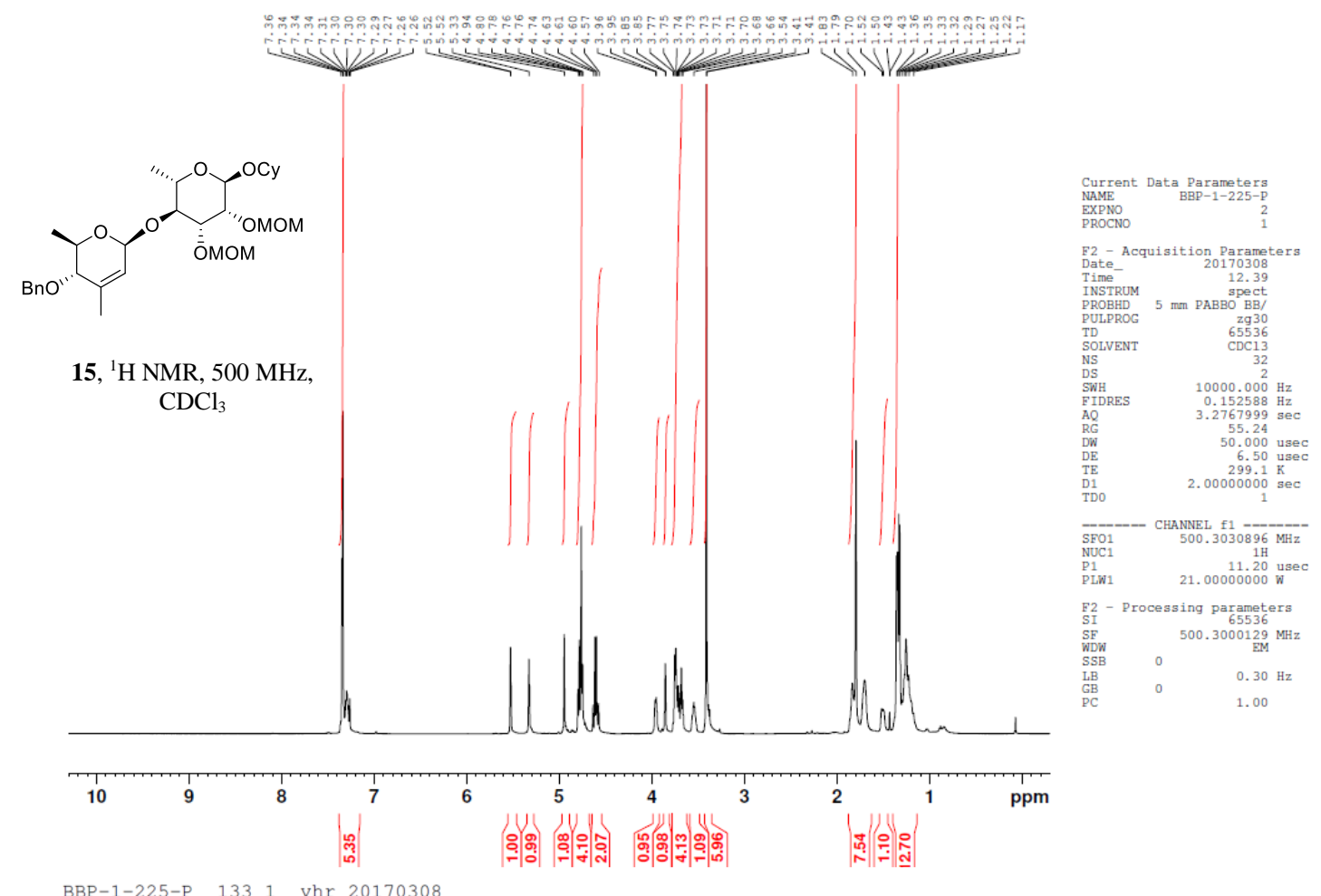

BBP-1-225-P 1331 yhr 20170308

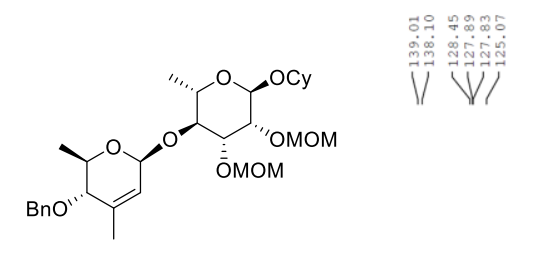

15, ${ }^{13} \mathrm{C}$ NMR, 125 $\mathrm{MHz}, \mathrm{CDCl}_{3}$
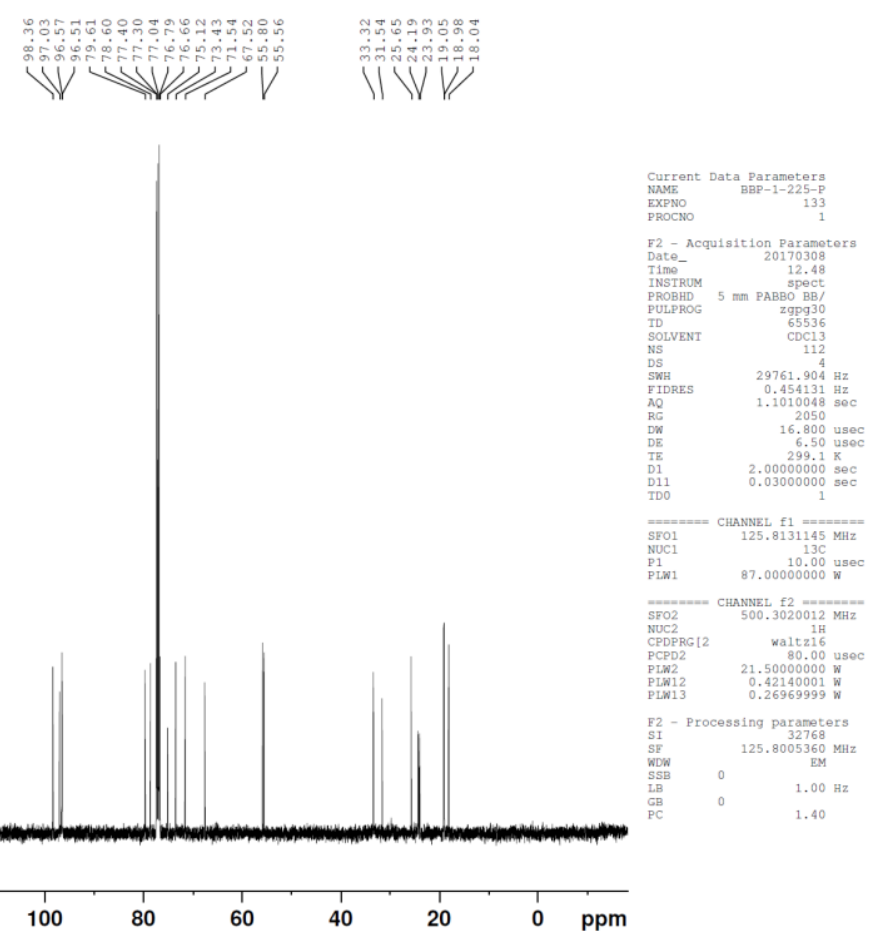


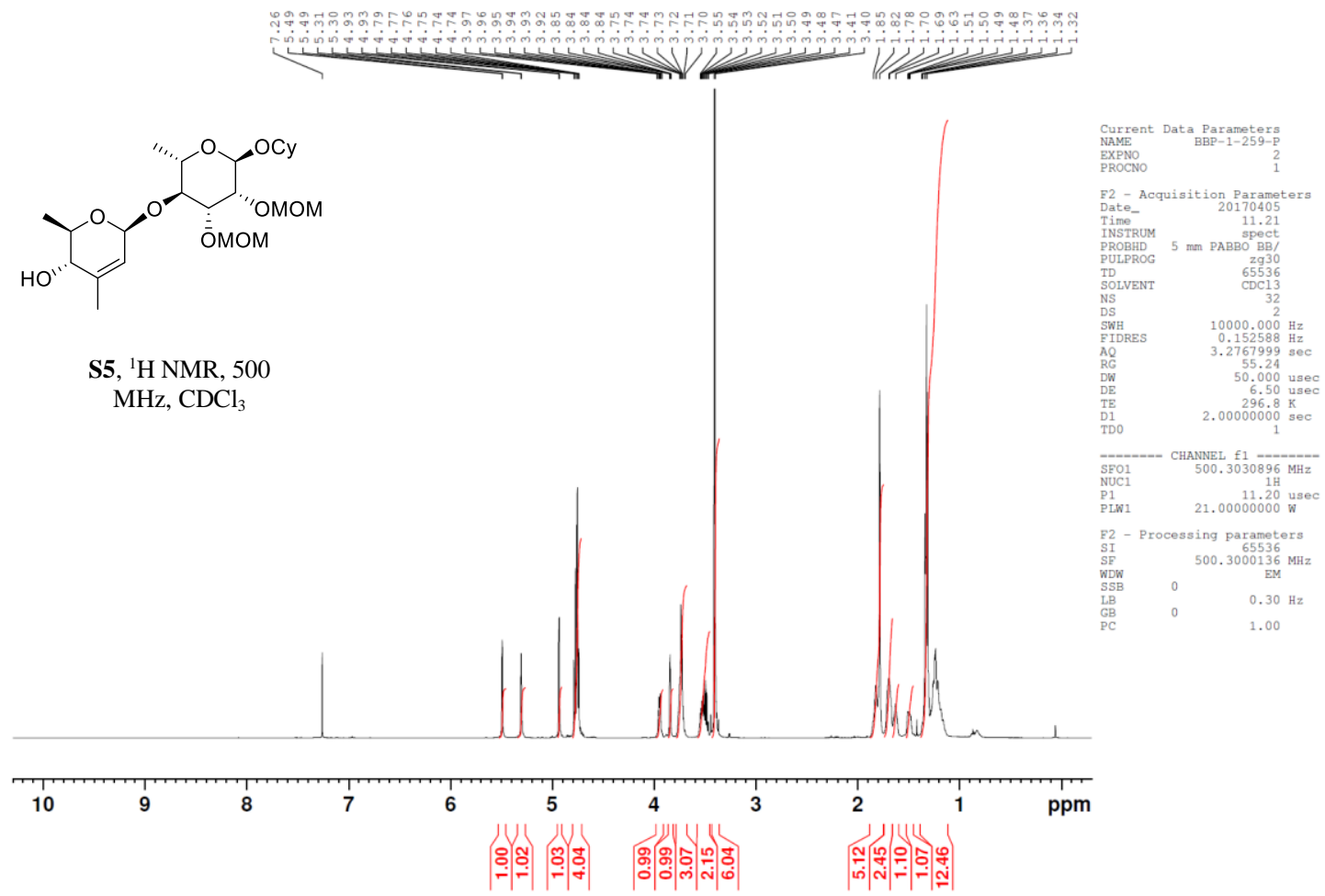

BBP-1-259-P 131 yhr 20170404

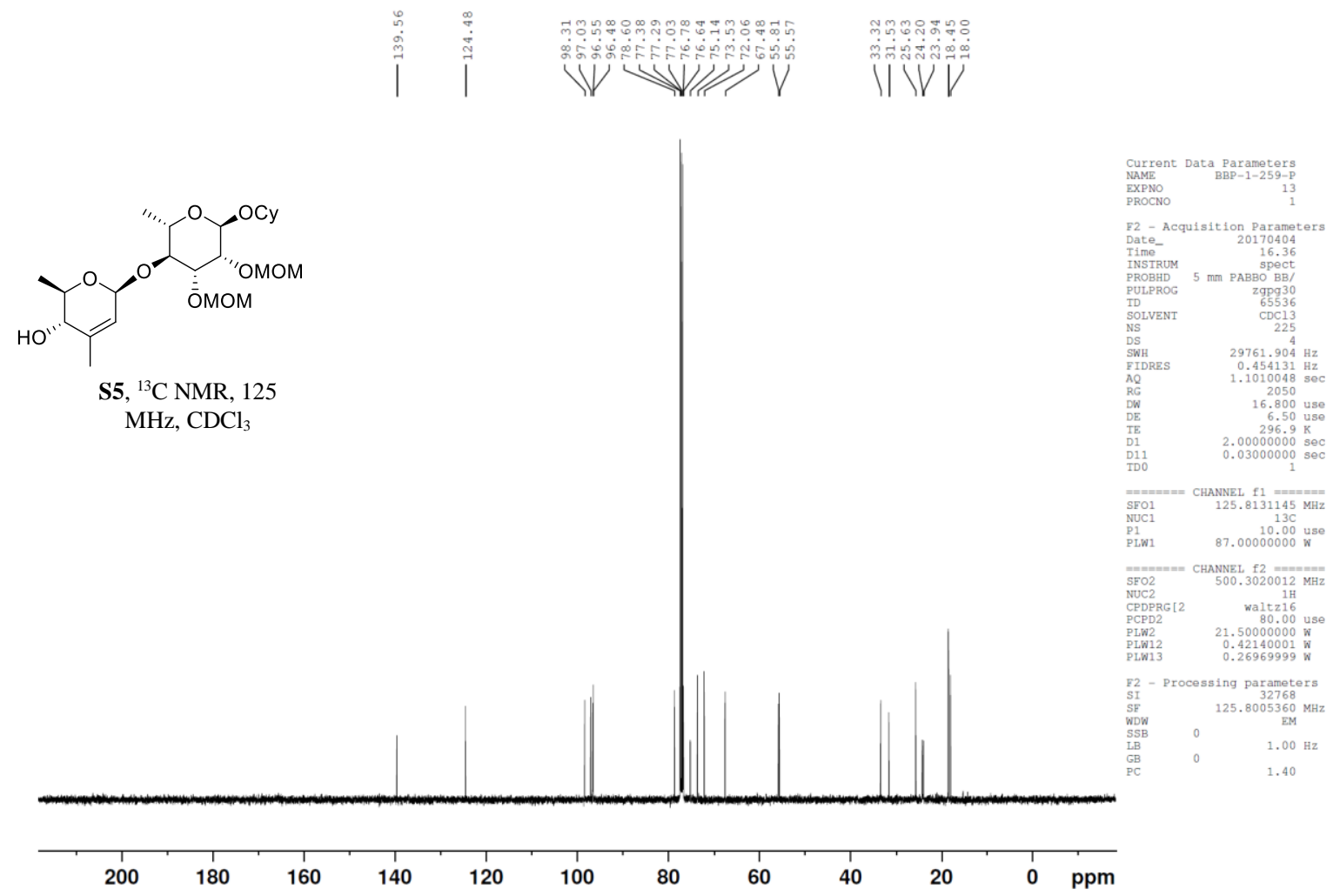




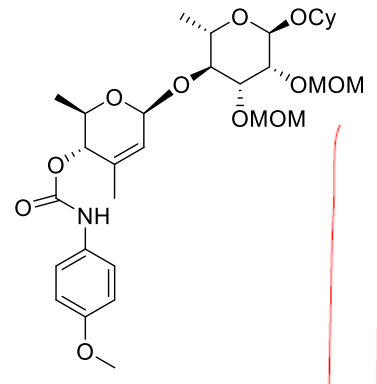

16, ${ }^{1} \mathrm{H} \mathrm{NMR}, 500 \mathrm{MHz}$, $\mathrm{CDCl}_{3}$
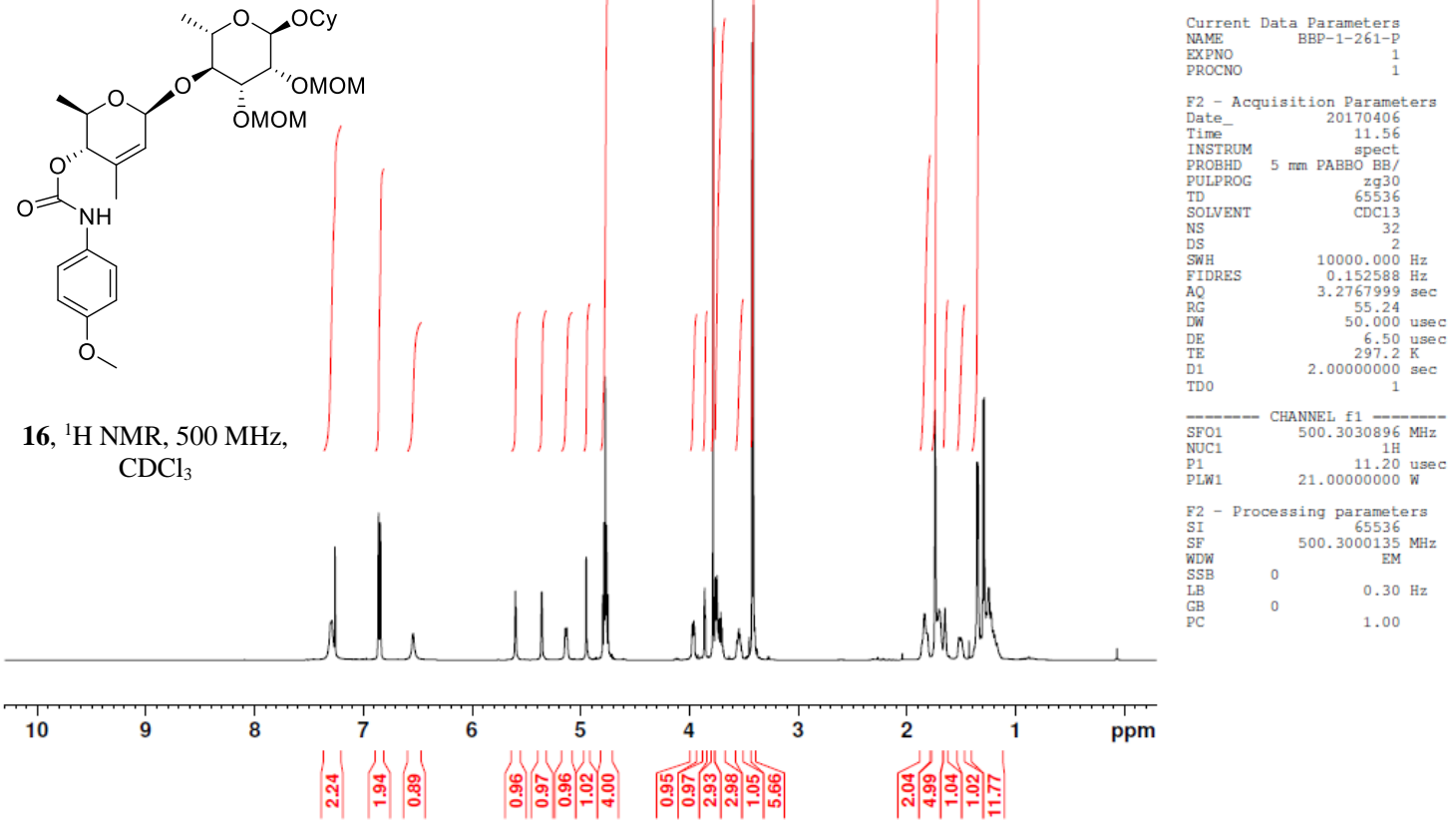

BBP-1-261-P-041117
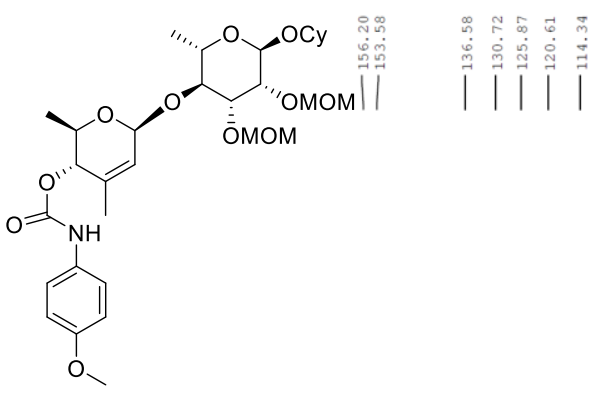

16, ${ }^{13} \mathrm{C}$ NMR, $150 \mathrm{MHz}$,

$\mathrm{CDCl}_{3}$
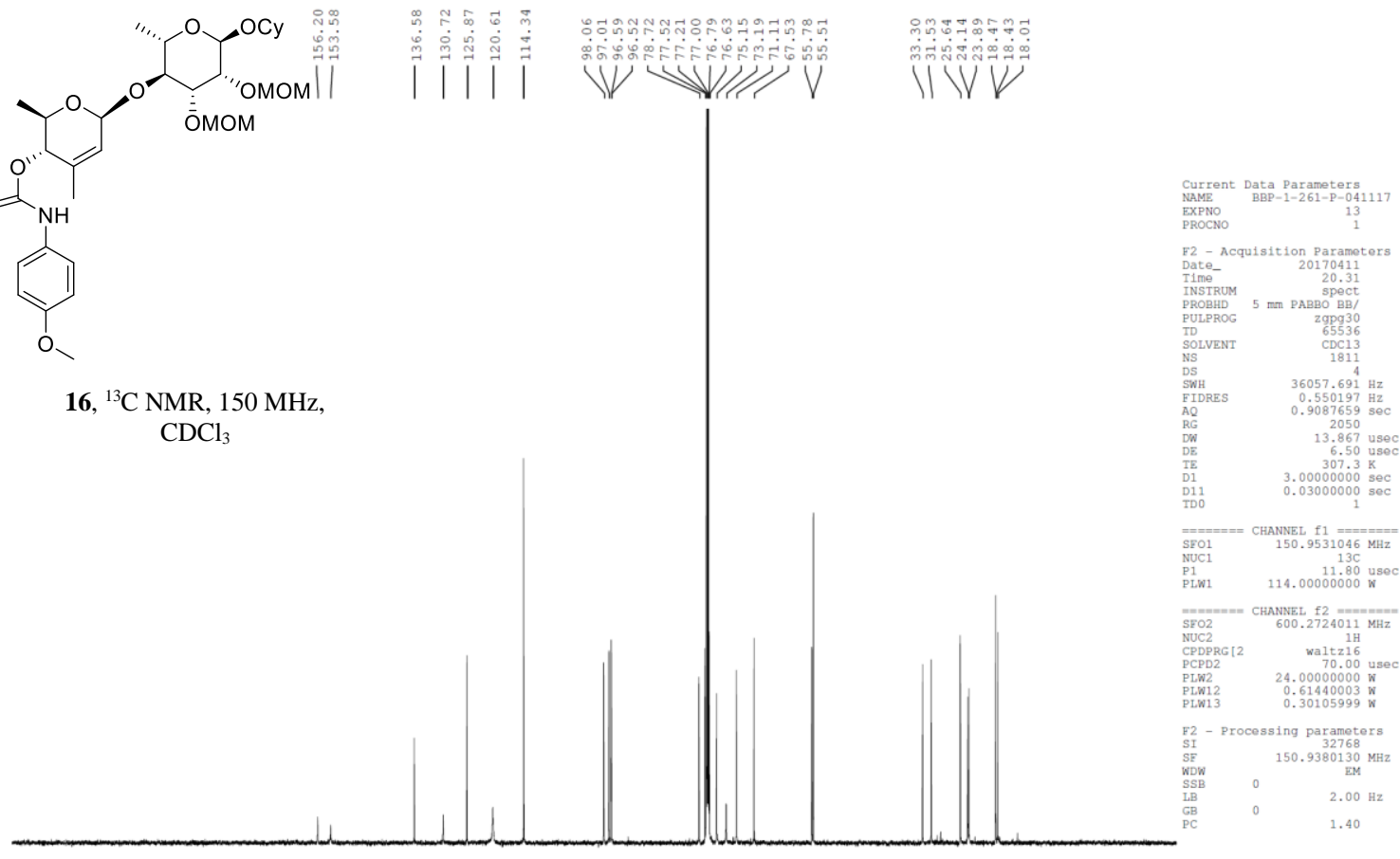


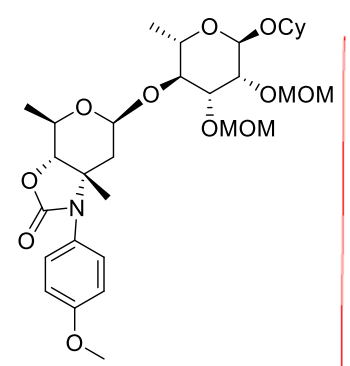

17, ${ }^{1} \mathrm{H}$ NMR, $500 \mathrm{MHz}$, $\mathrm{CDCl}_{3}$

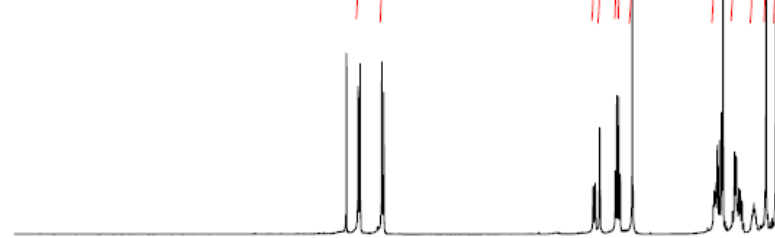

$\begin{array}{llllll}10 & 9 & 8 & 7 & 6\end{array}$

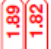

BBP-1-267-P $134 \quad 120170415$

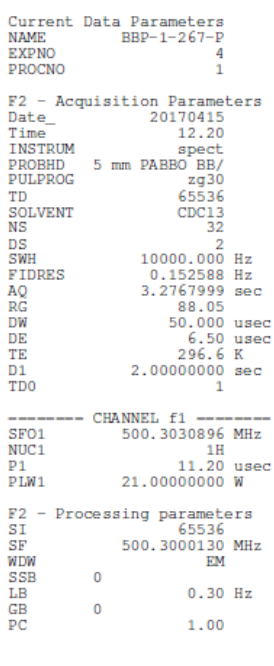

$\mathrm{pm}$
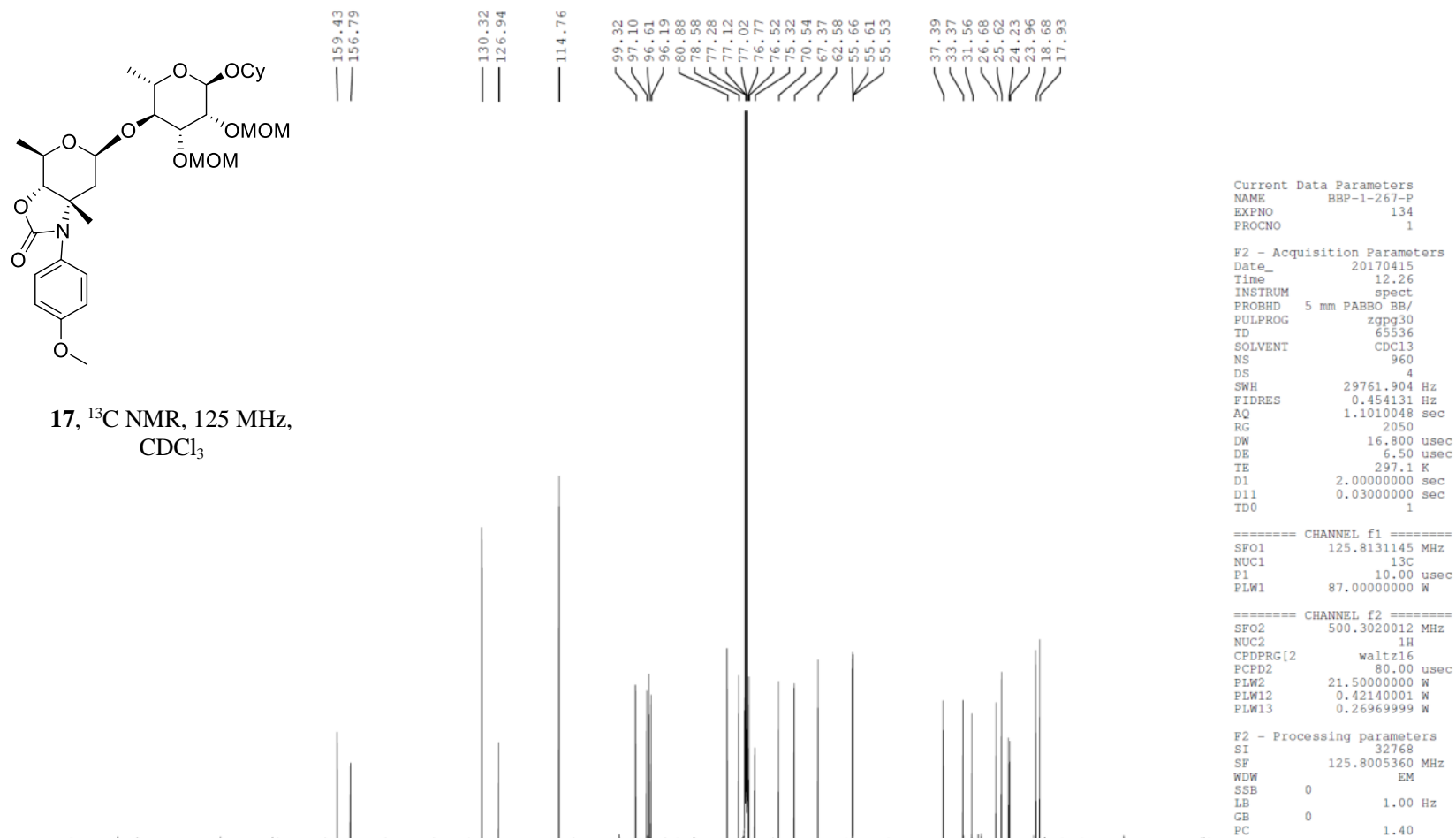

17, ${ }^{13} \mathrm{C}$ NMR, $125 \mathrm{MHz}$

$\mathrm{CDCl}_{3}$
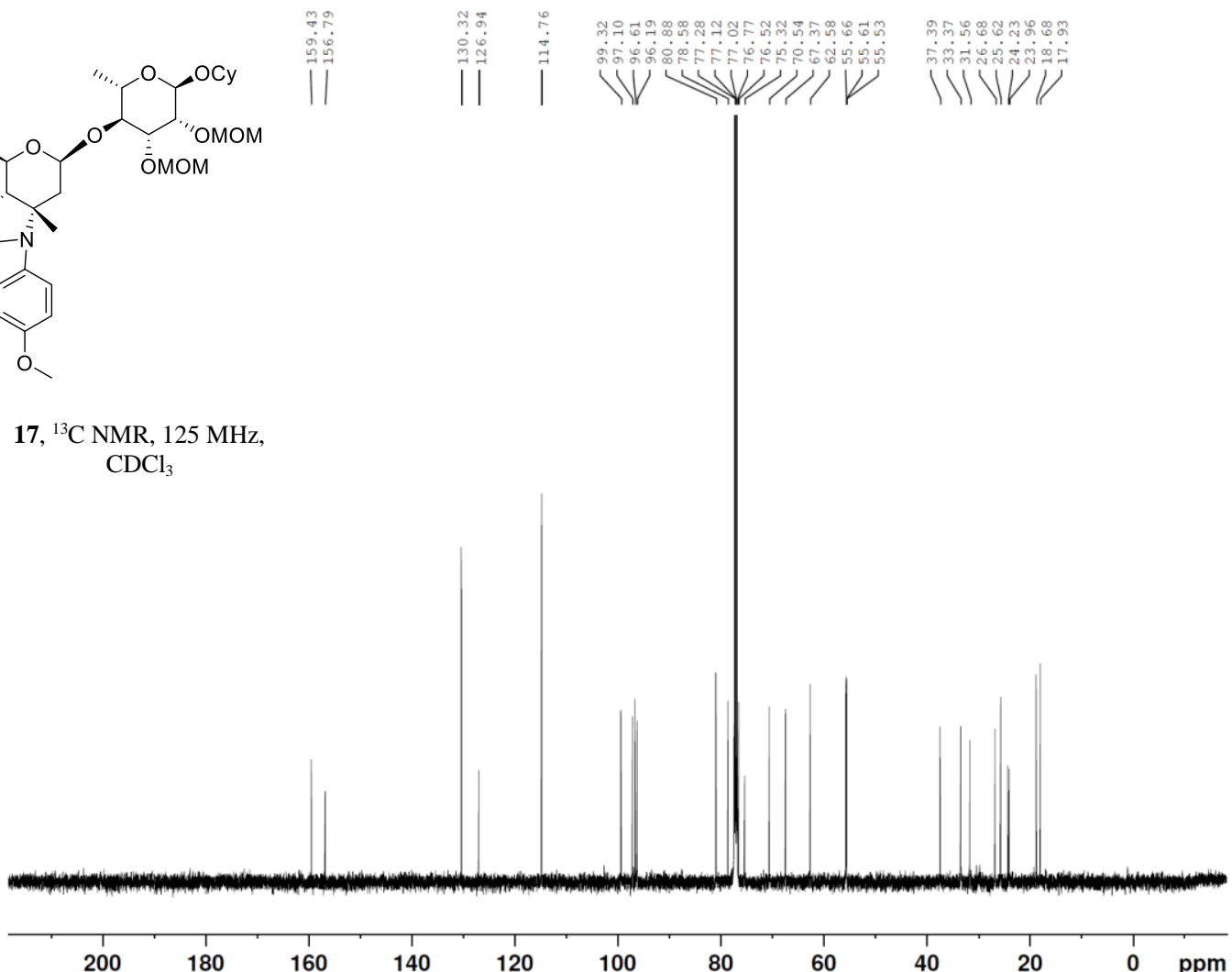


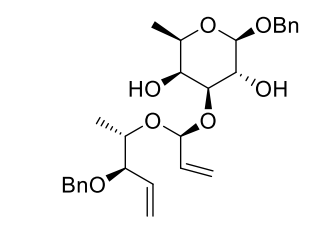

S6, ${ }^{1} \mathrm{H}$ NMR, $500 \mathrm{MHz}$, $\mathrm{CDCl}_{3}$
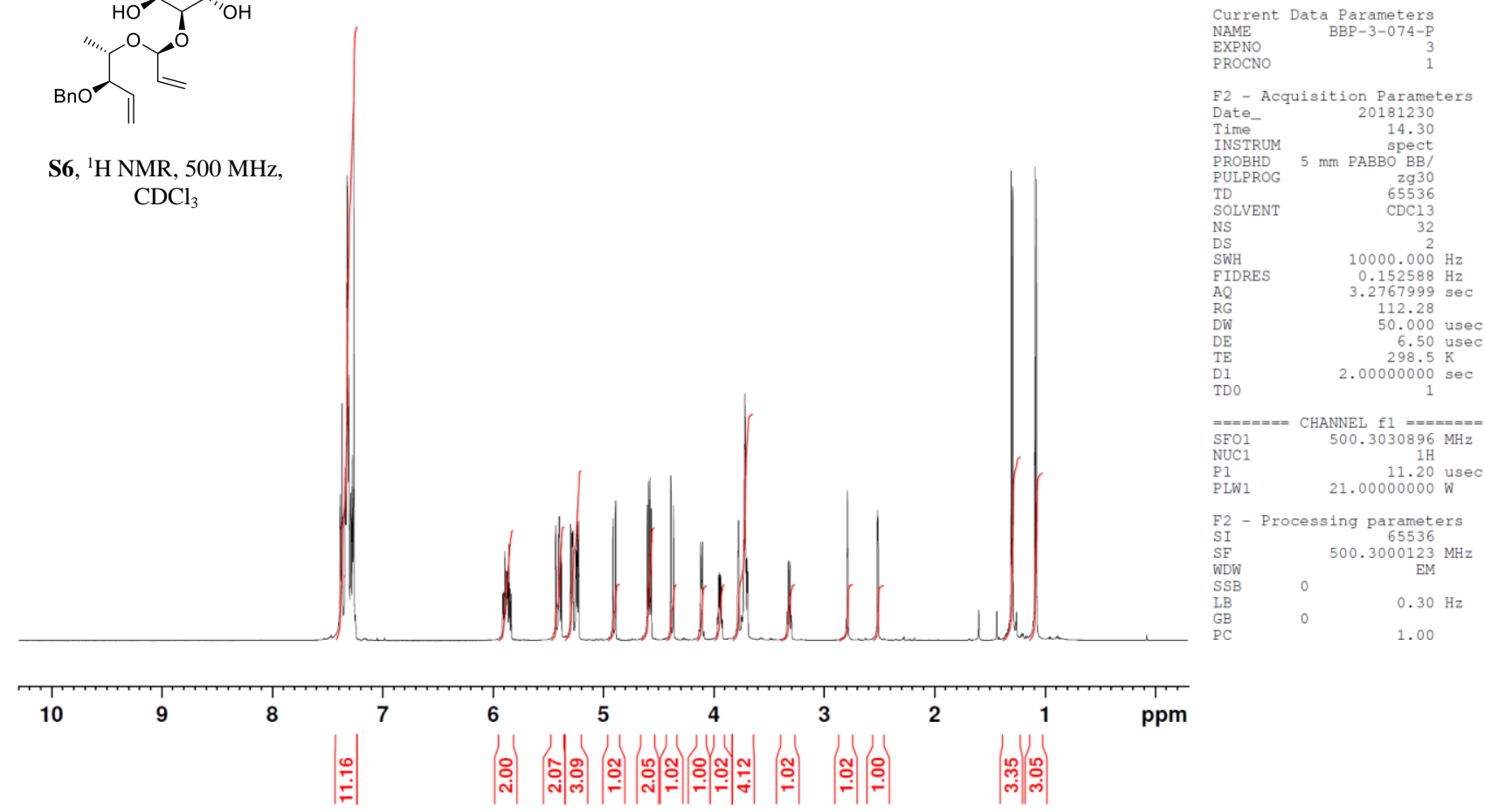

BBP-3-074-P 132120181230

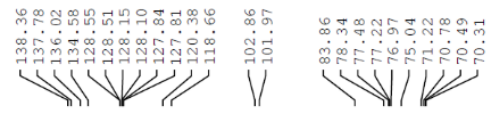

Vิ

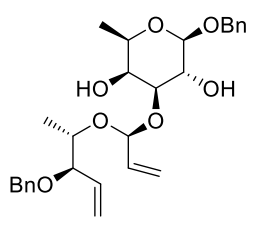

S6, ${ }^{13} \mathrm{C}$ NMR, 125

$\mathrm{MHz}, \mathrm{CDCl}_{3}$
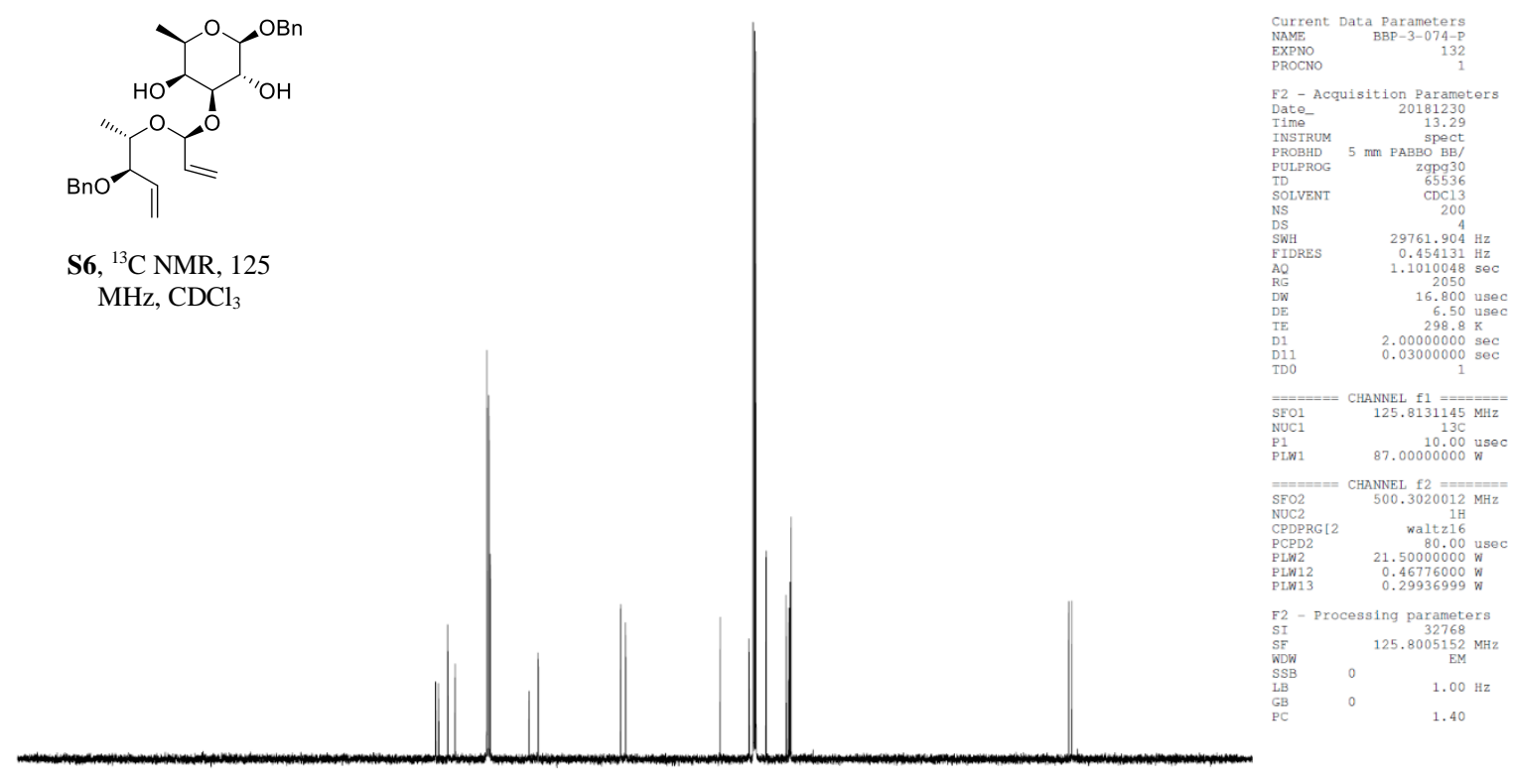

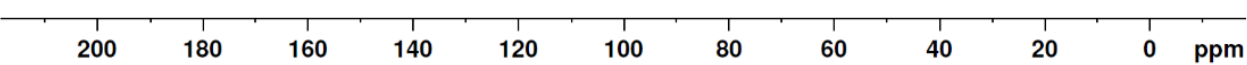




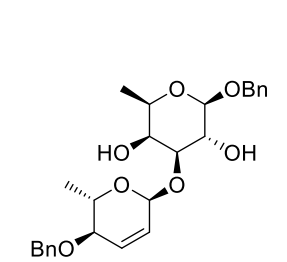

21a, ${ }^{1} \mathrm{H}$ NMR, $500 \mathrm{MHz}$, $\mathrm{CDCl}_{3}$
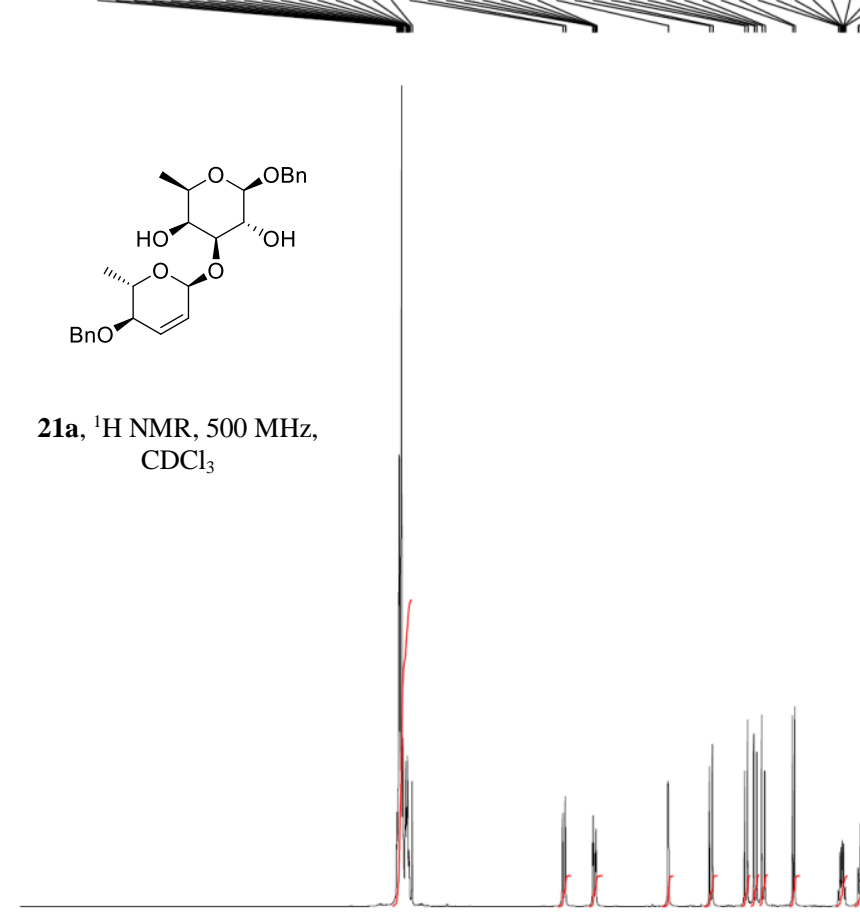

109

8
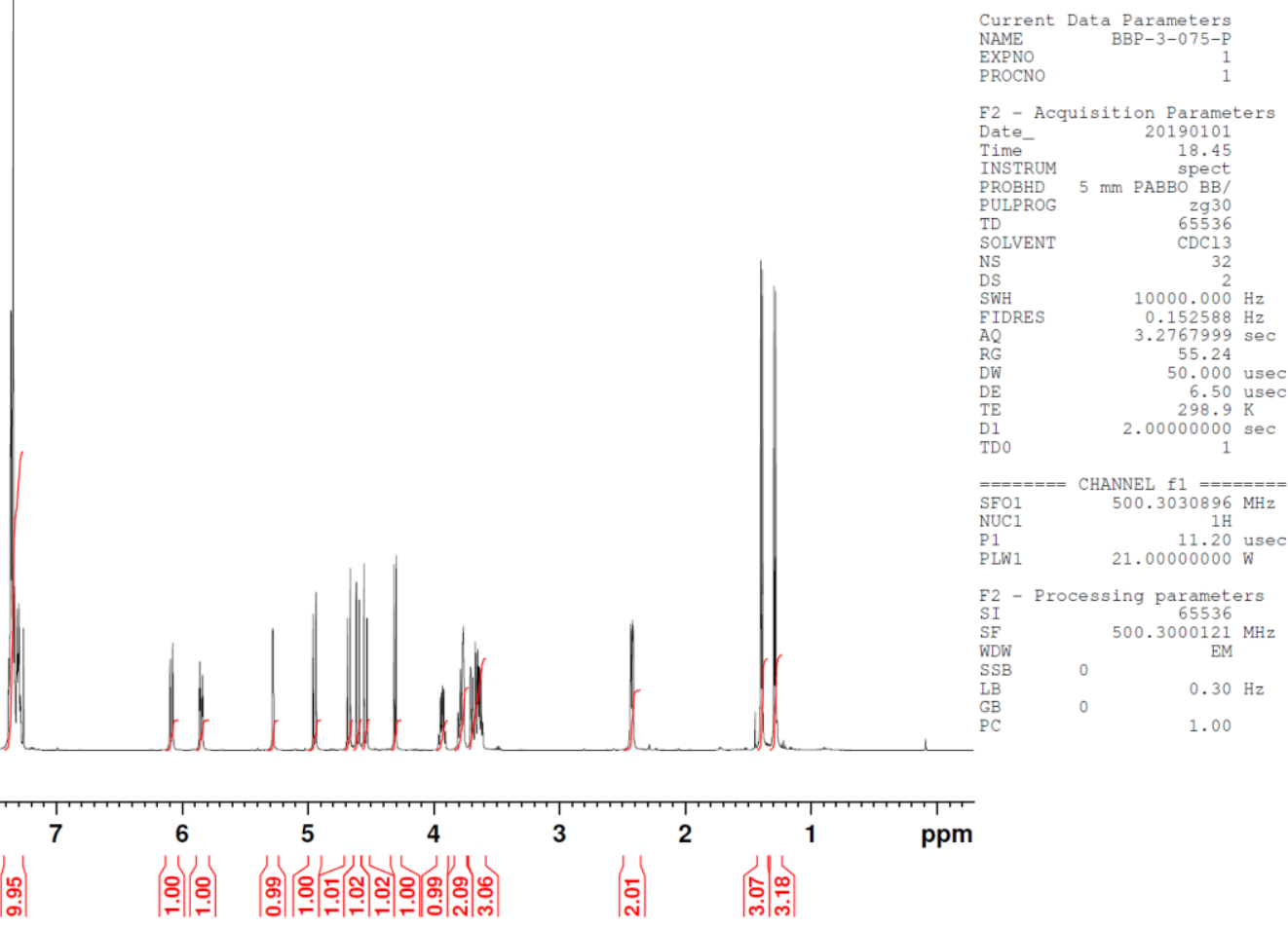

BBP-3-075-P 13120190101
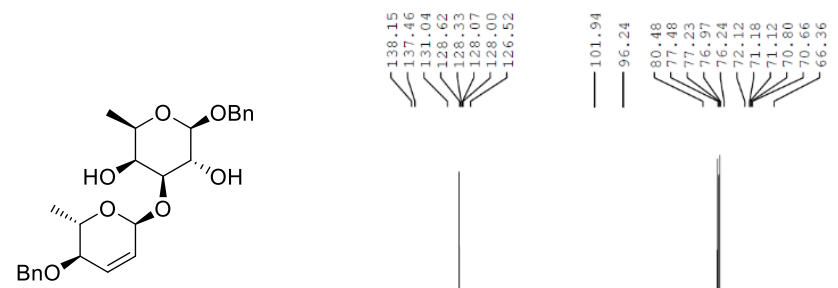

$\mid$

21a, ${ }^{13} \mathrm{C}$ NMR, 125 $\mathrm{MHz}, \mathrm{CDCl}_{3}$
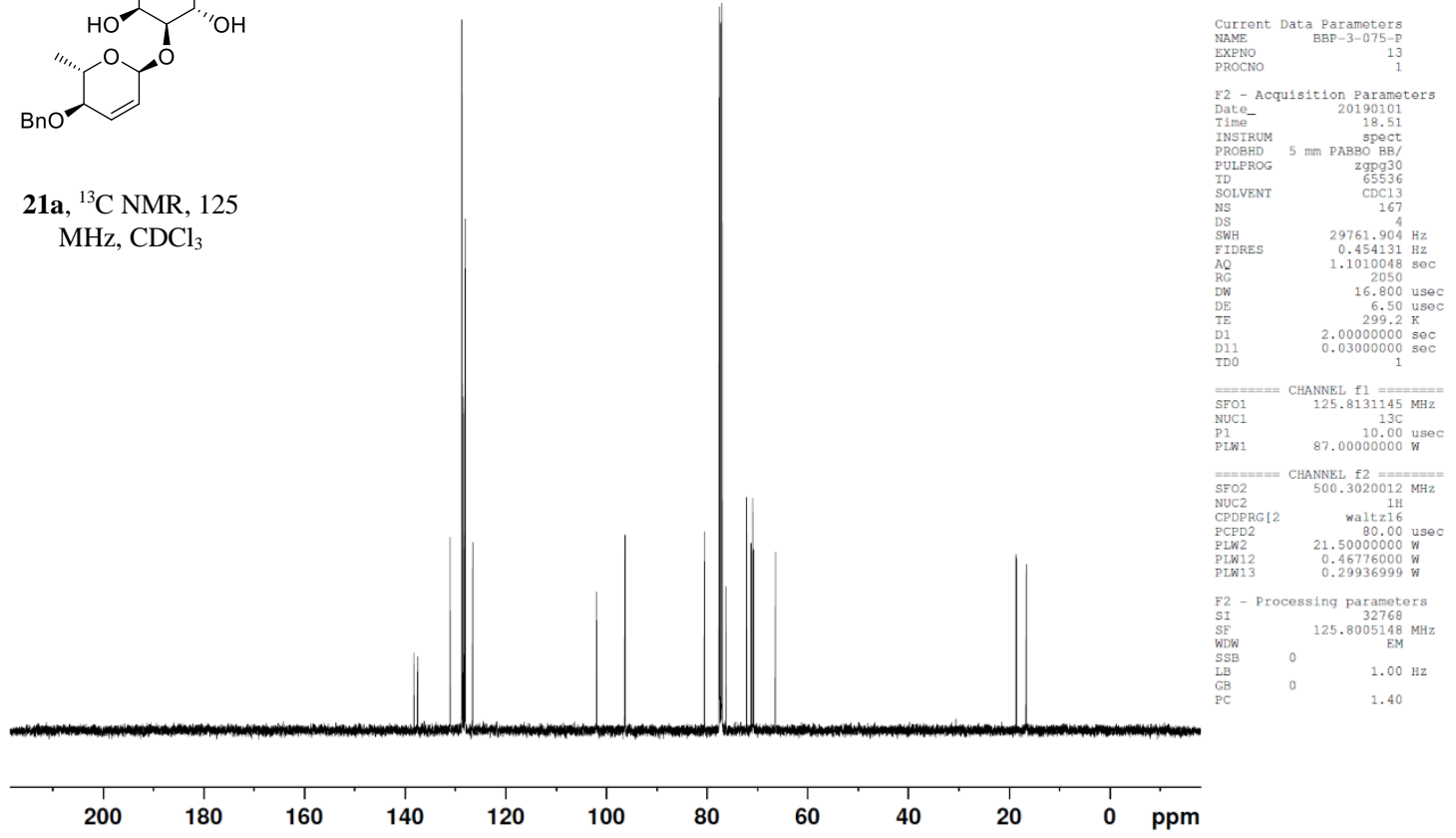


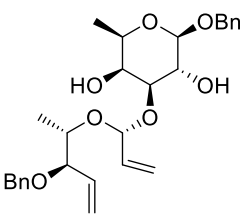

S7, ${ }^{1} \mathrm{H}$ NMR, $500 \mathrm{MHz}$, $\mathrm{CDCl}_{3}$
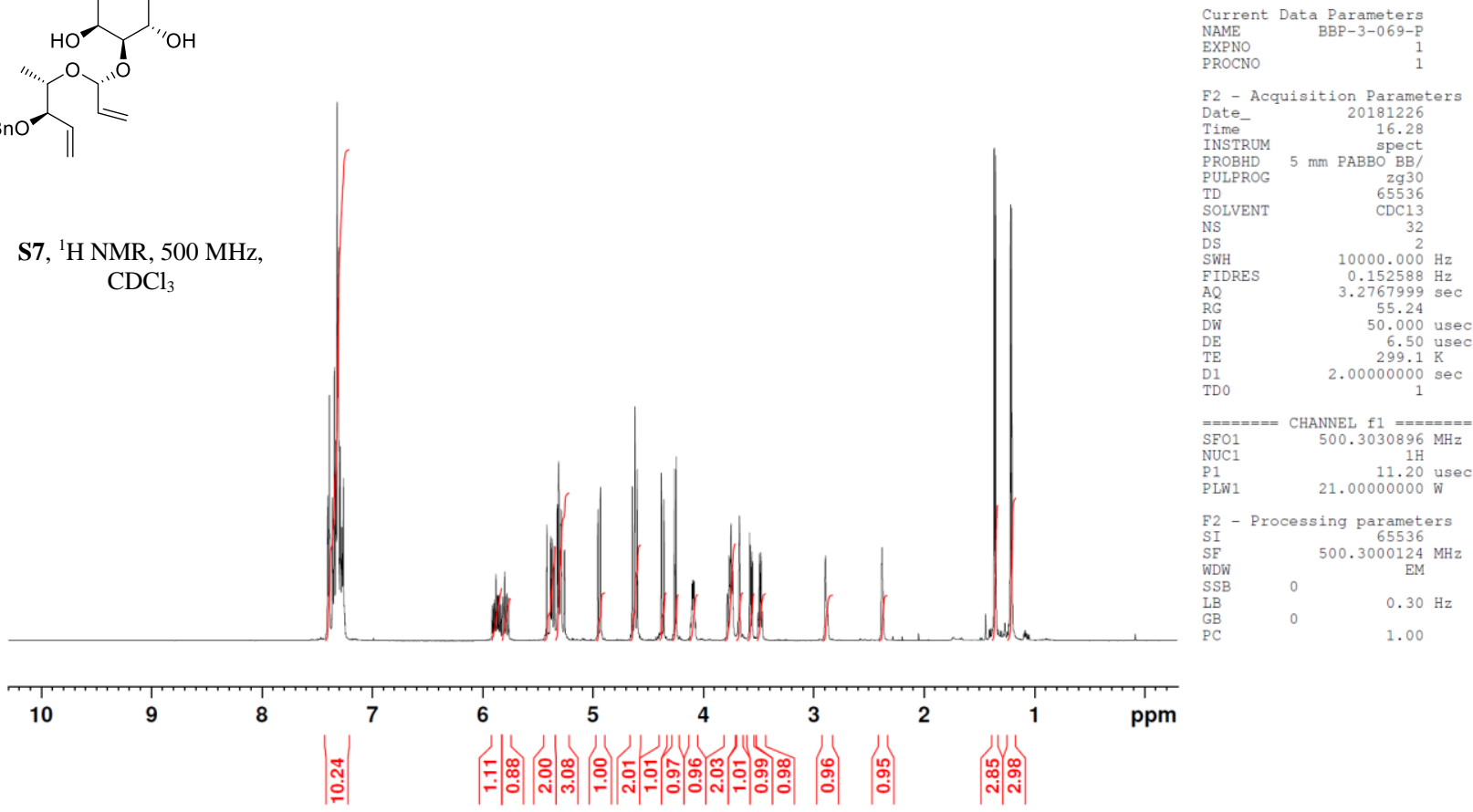

BBP-3-069-P 13120181226
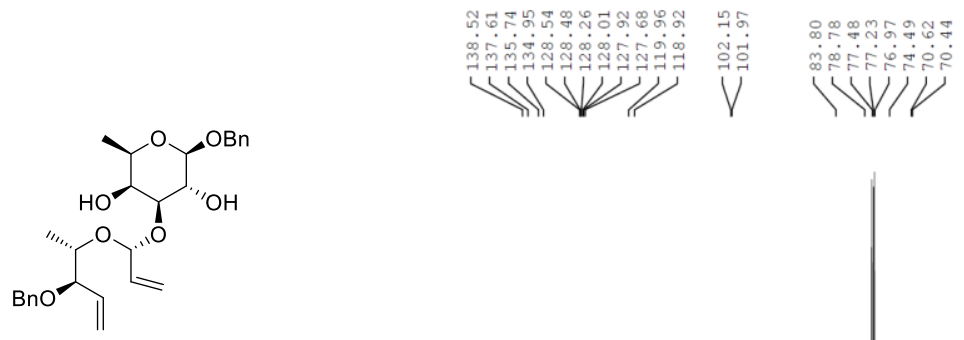

Vั้

S7, ${ }^{13} \mathrm{C}$ NMR, $125 \mathrm{MHz}$,

$\mathrm{CDCl}_{3}$
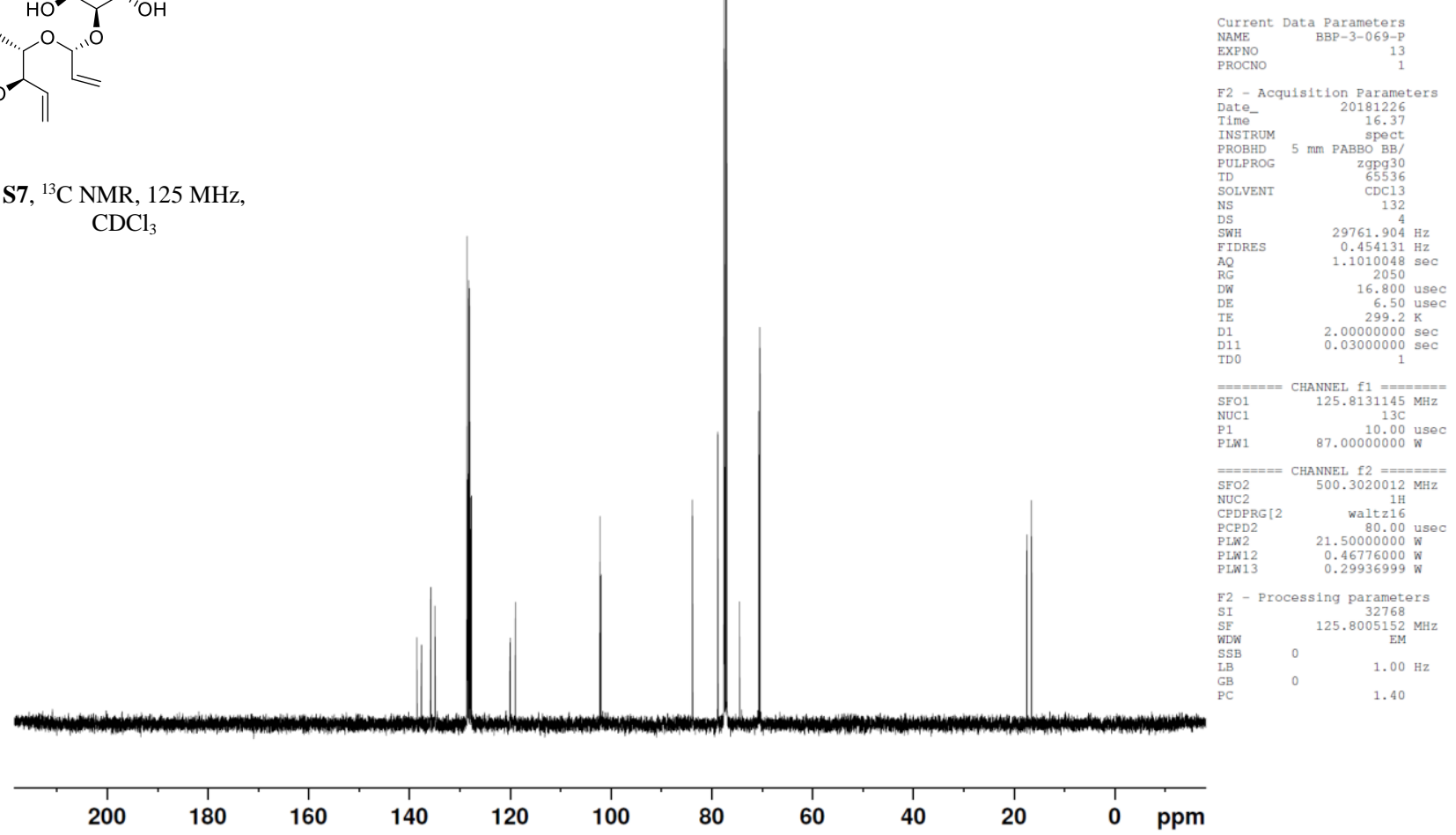


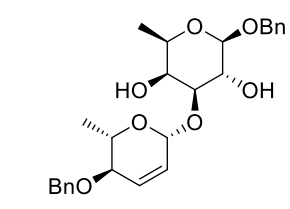

21b, ${ }^{1} \mathrm{H}$ NMR, $500 \mathrm{MHz}$, $\mathrm{CDCl}_{3}$

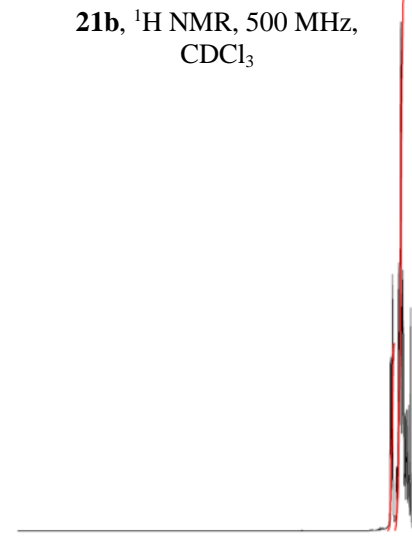

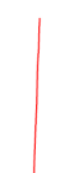

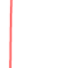

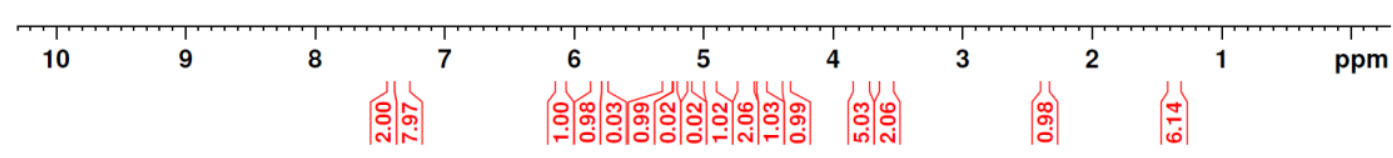

BBP-3-072-P 13120181226
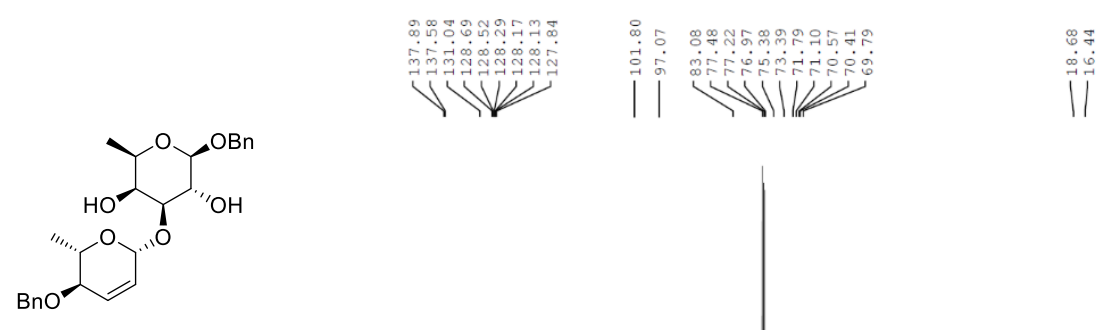

21b, ${ }^{13} \mathrm{C}$ NMR, 125 $\mathrm{MHz}, \mathrm{CDCl}_{3}$
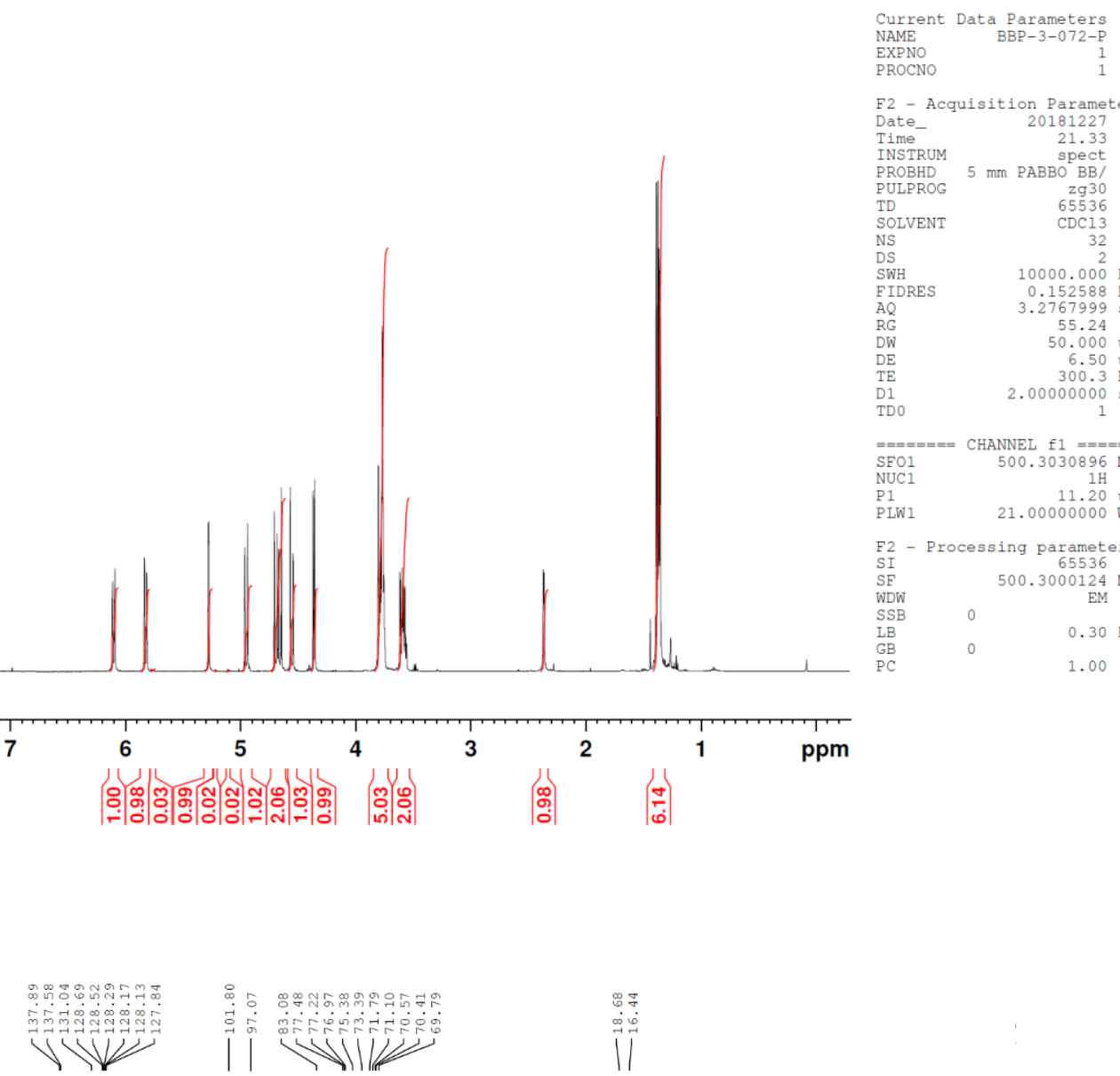

PROCNO

F2 - Acquisition Parameters

Date Acquisition paramet

$\begin{array}{lr}\text { Time- } & 21.33\end{array}$

spect
PROBHD $5 \mathrm{~mm}$ PABBO BB/

$\begin{array}{lr}\text { PULPROG } & \text { zg30 } \\ \text { TD } & 65536 \\ \text { SOLVENT } & \text { CDC13 } \\ \text { NS } & 32\end{array}$

NS

SWH $\quad 10000.000 \mathrm{~Hz}$

$\begin{array}{ll}\text { SWH } & 10000.000 \mathrm{~Hz} \\ \text { FIDRES } & 0.152588 \mathrm{~Hz}\end{array}$

$\begin{array}{lr}\text { AQ } & 3.2767999 \mathrm{sec} \\ \text { RG } & 55.24 \\ \text { DW } & 50.000 \mathrm{usec}\end{array}$

$\begin{array}{lr}\text { DW } & 50.000 \text { usec } \\ \text { DE } & 6.50 \text { usec }\end{array}$

$300.3 \mathrm{~K}$
TE

$\begin{array}{lr}\text { D1 } & 2.00000000 \\ \text { TD } & \text { sec }\end{array}$

$======0$ CHANNEL $\mathrm{f} 1=======$

$\begin{array}{lr}\text { SFO1 } & 500.3030896 \mathrm{MHz} \\ \text { NUC1 } & 1 \mathrm{H}\end{array}$

$\begin{array}{lr}\text { P1 } & 11.20 \text { usec } \\ \text { PLW1 } & 21.00000000 \mathrm{~W}\end{array}$

F2 - Processing parameters

SI $\quad 500.3000124 \mathrm{MHz}$

WDW

$\begin{array}{ll}\text { SSB } & 0 \\ \text { LB } & 0.30 \mathrm{~Hz}\end{array}$

PC

1.00

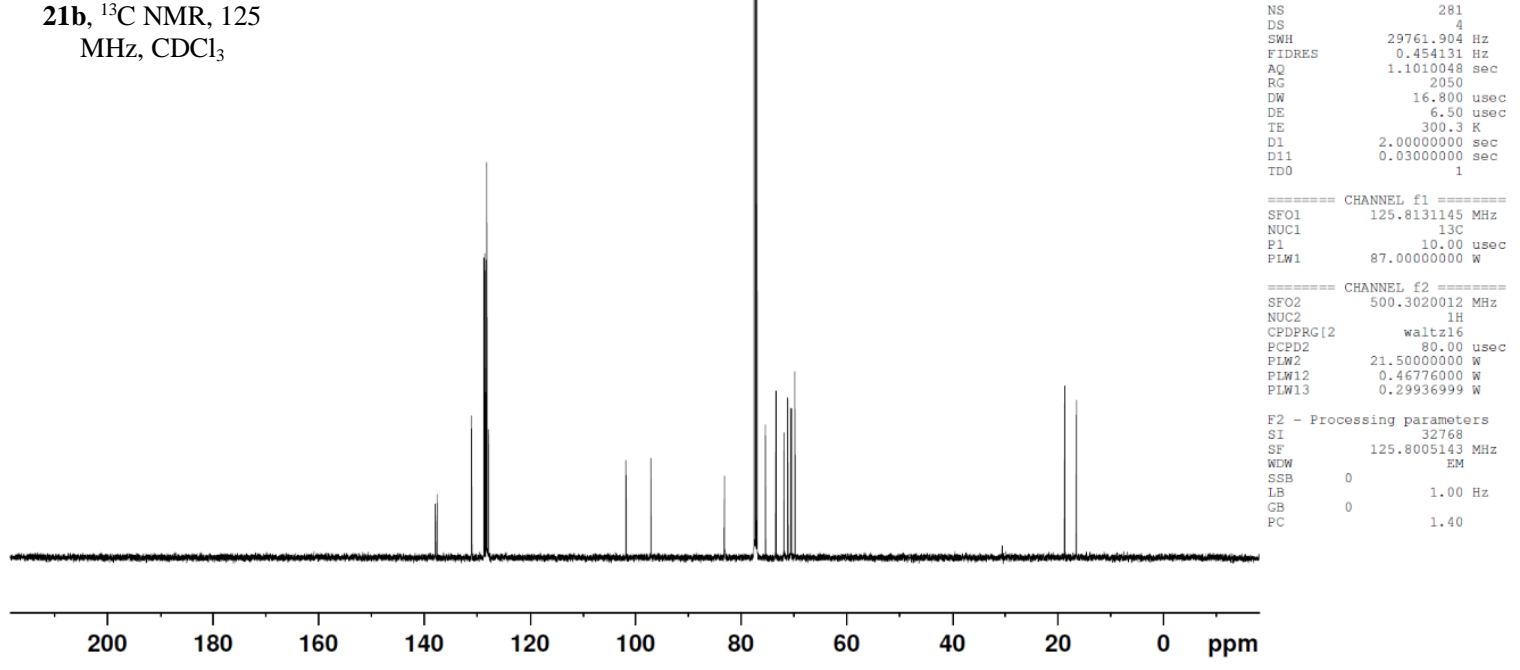



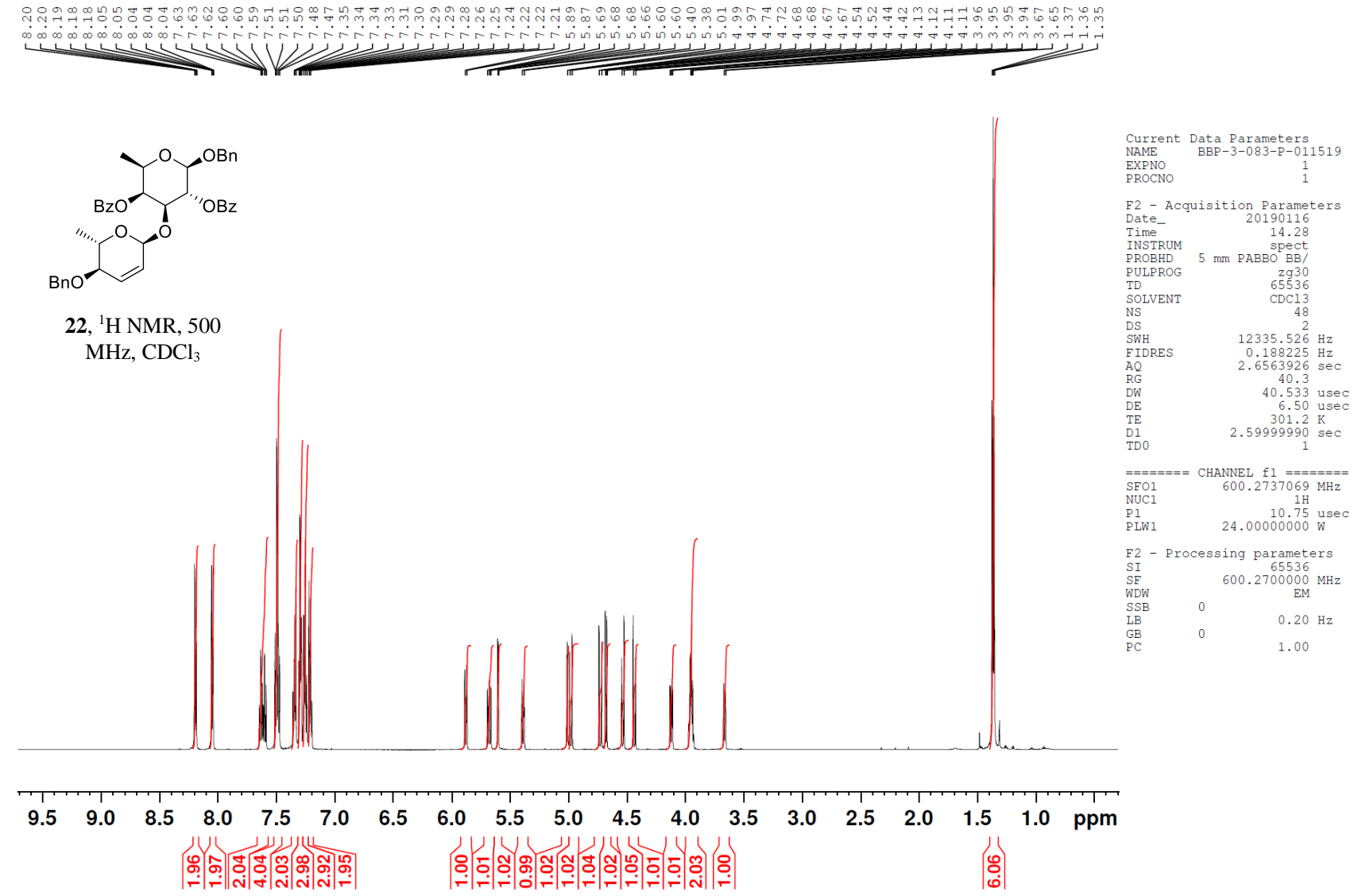

BBP-3-083-P 13120190109
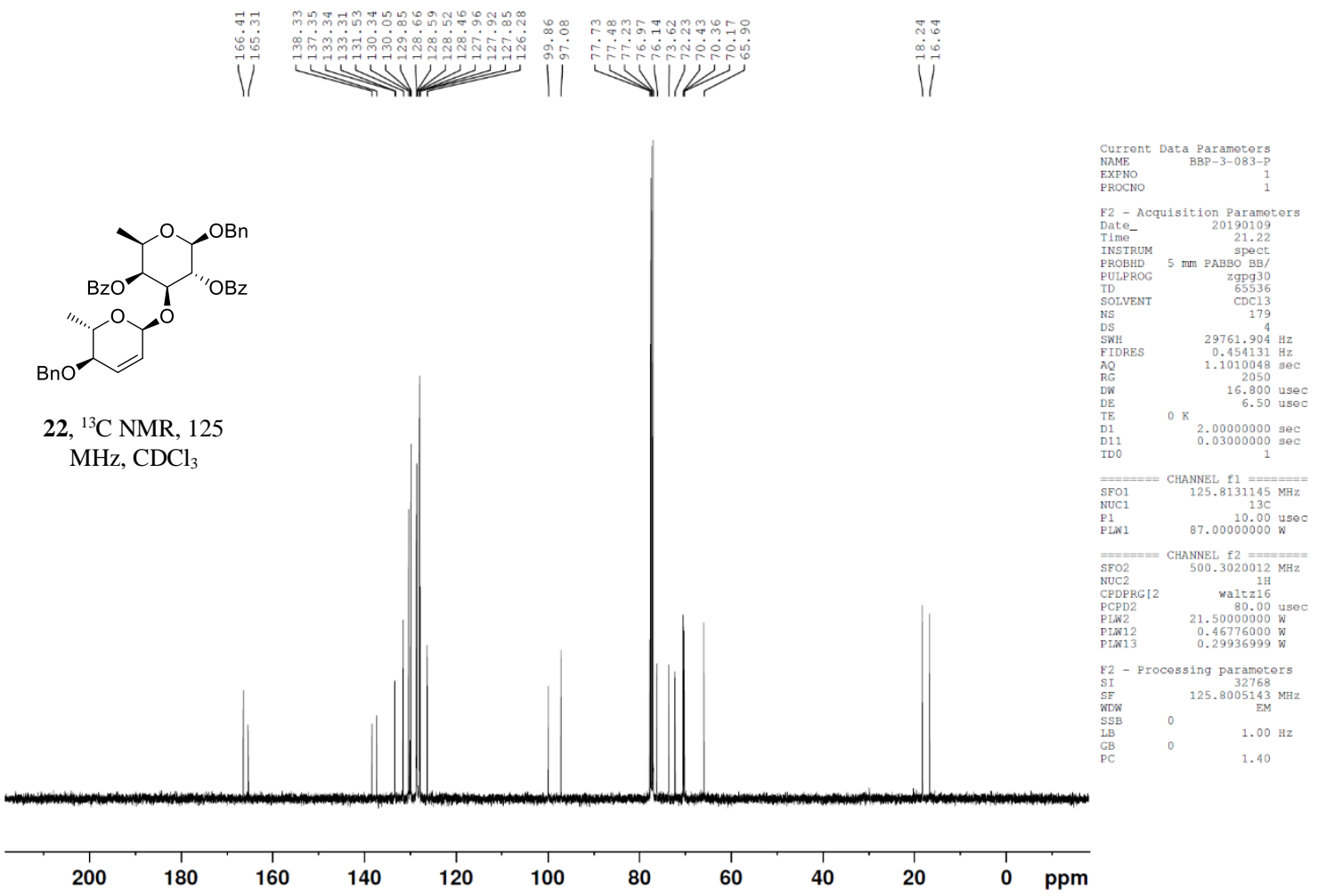


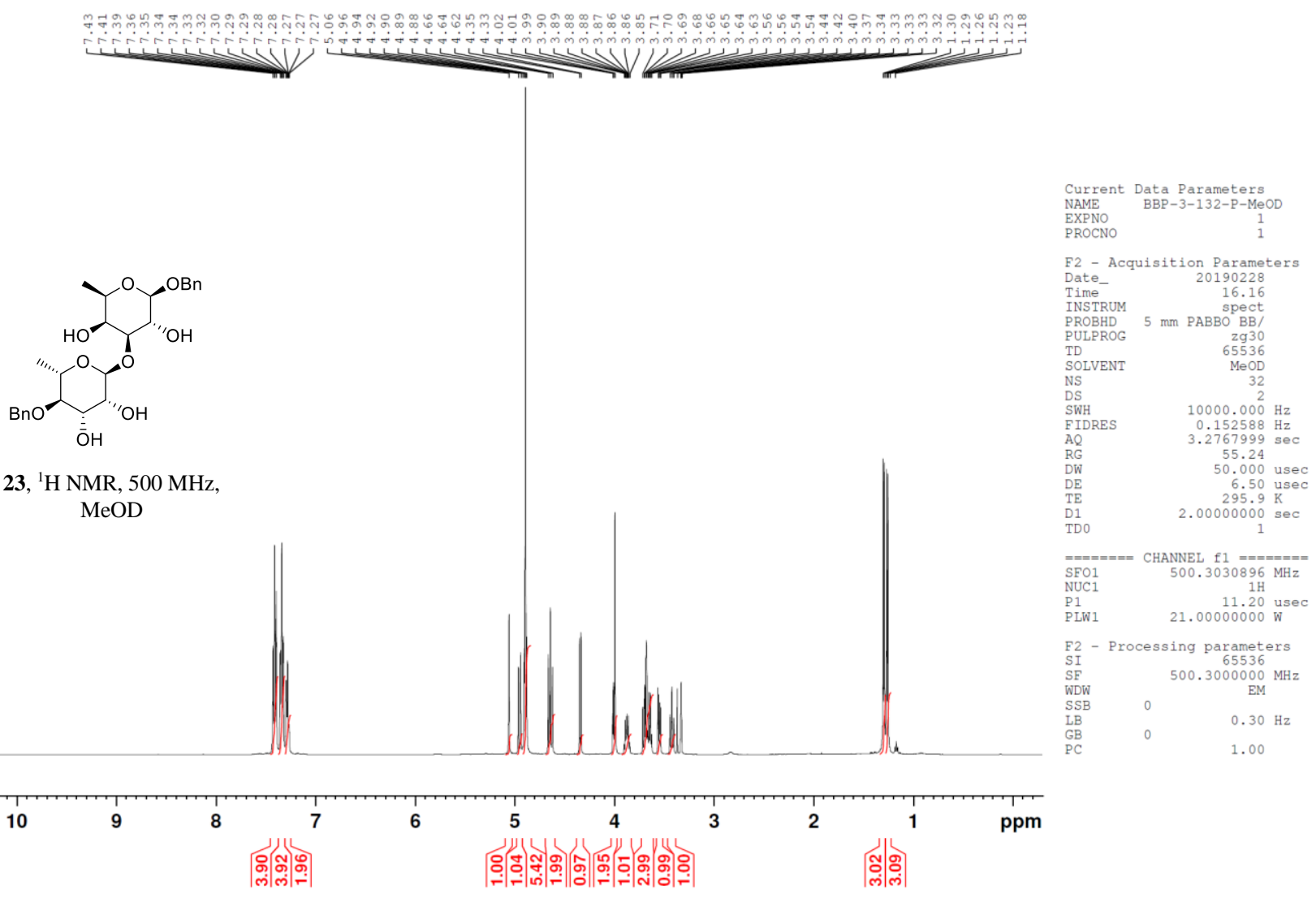<smiles>CC1OC([18O])C(O)C(OC2OC(C)[C@@H](O)[C@H](O)C2O)O1</smiles>

23, ${ }^{13} \mathrm{C}$ NMR, $125 \mathrm{MHz}$, $\mathrm{MeOD}$
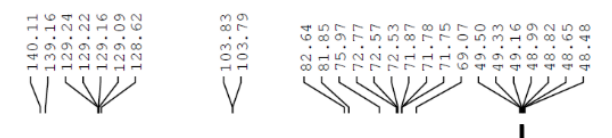

||
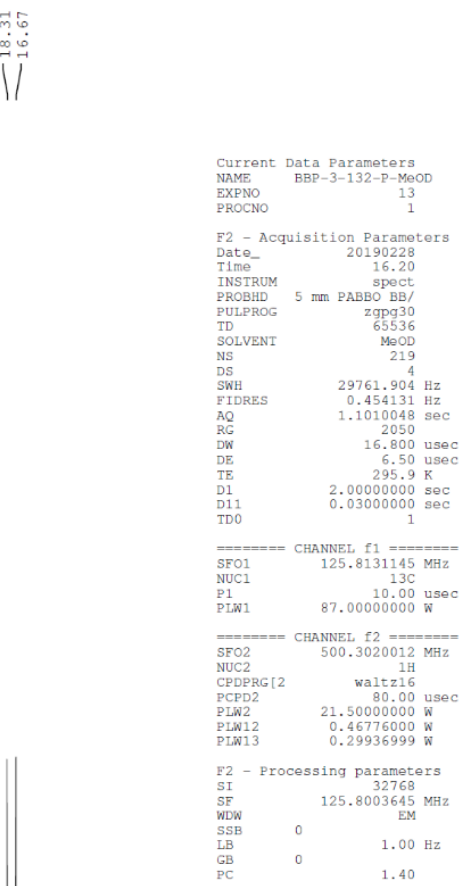


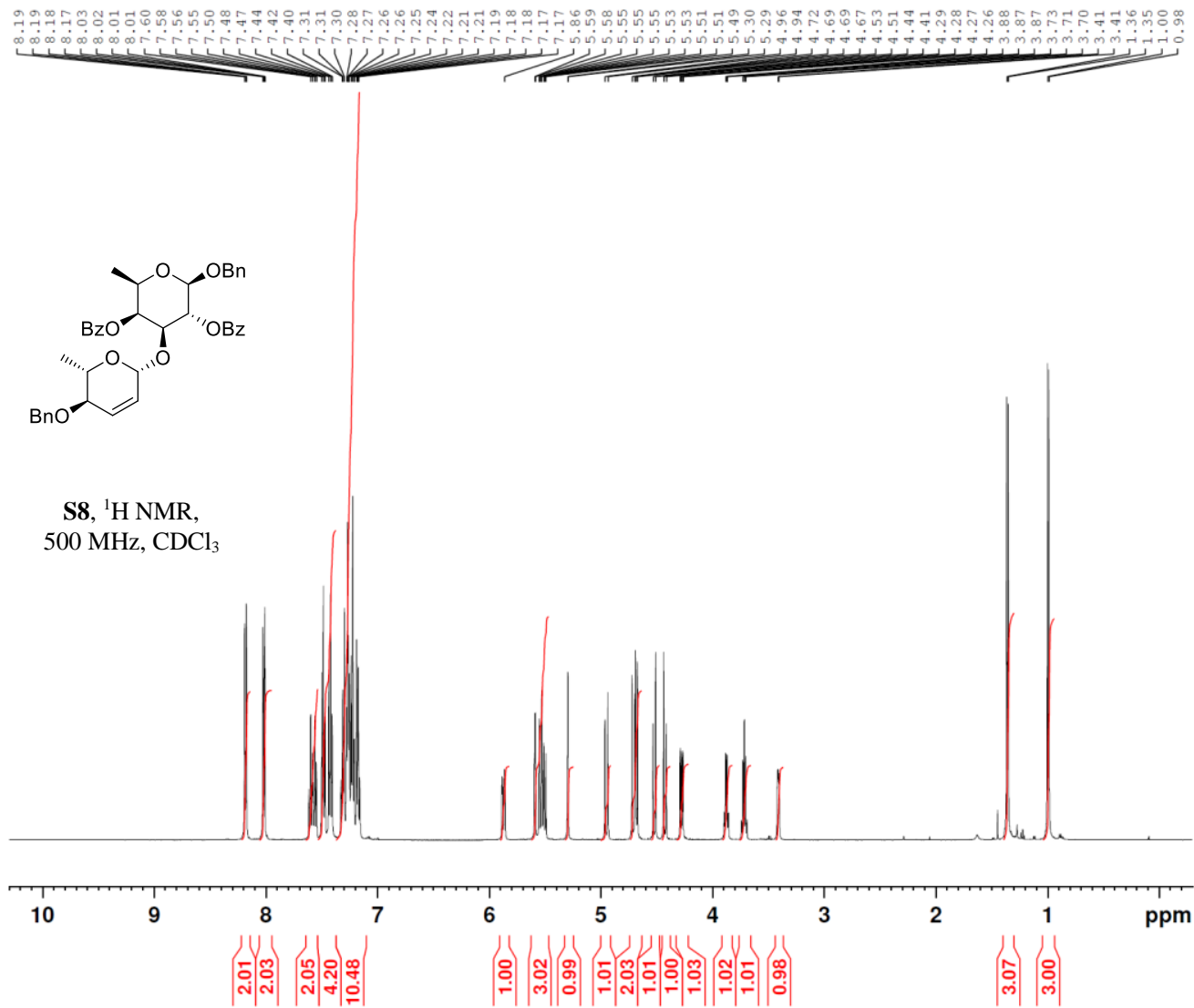

BBP-3-082-P 13120190109
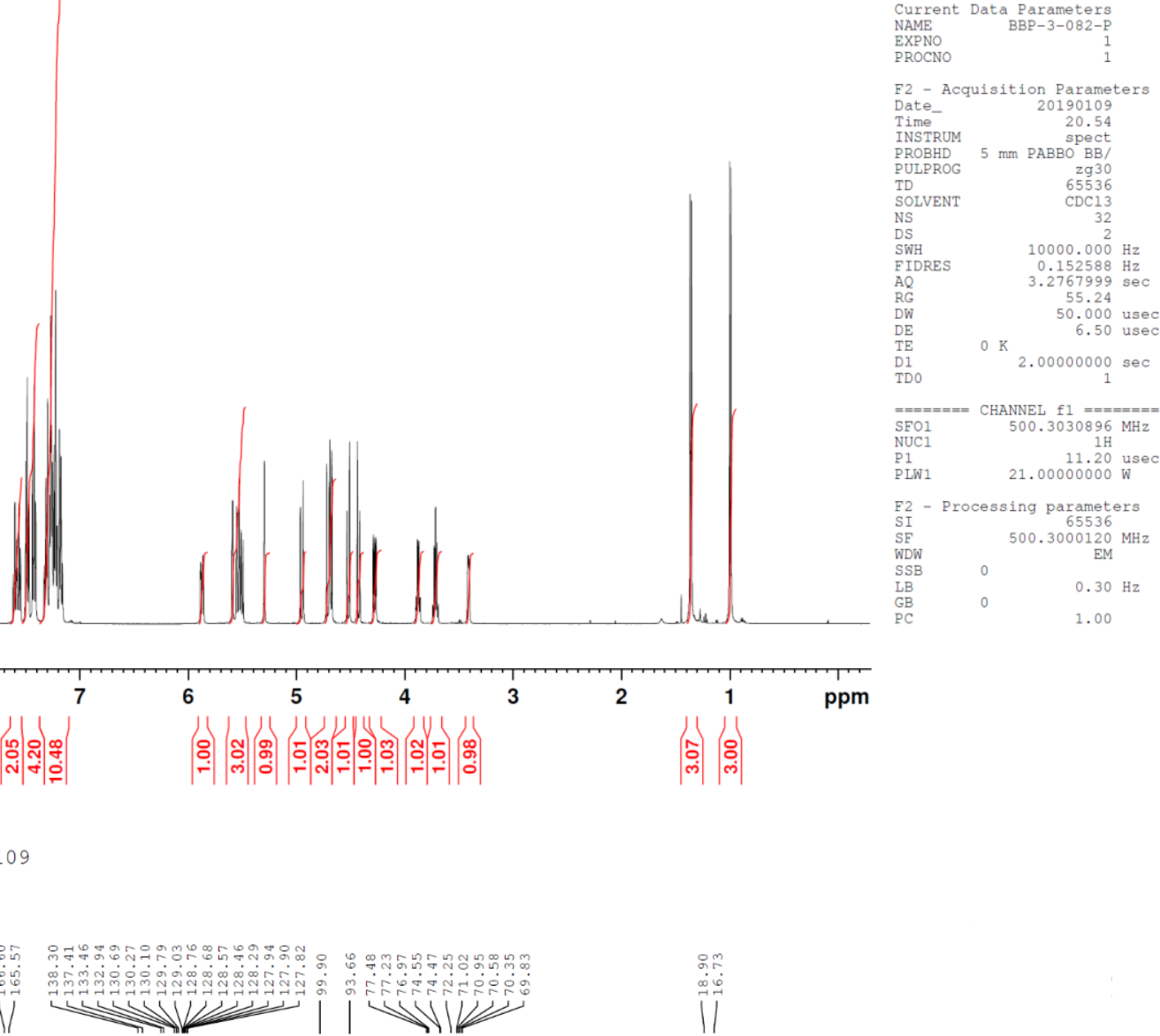

$1 \mid$

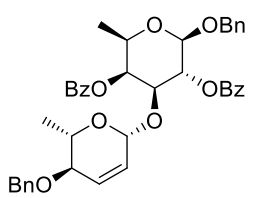

S8, ${ }^{13} \mathrm{C}$ NMR, 125

$\mathrm{MHz}, \mathrm{CDCl}_{3}$
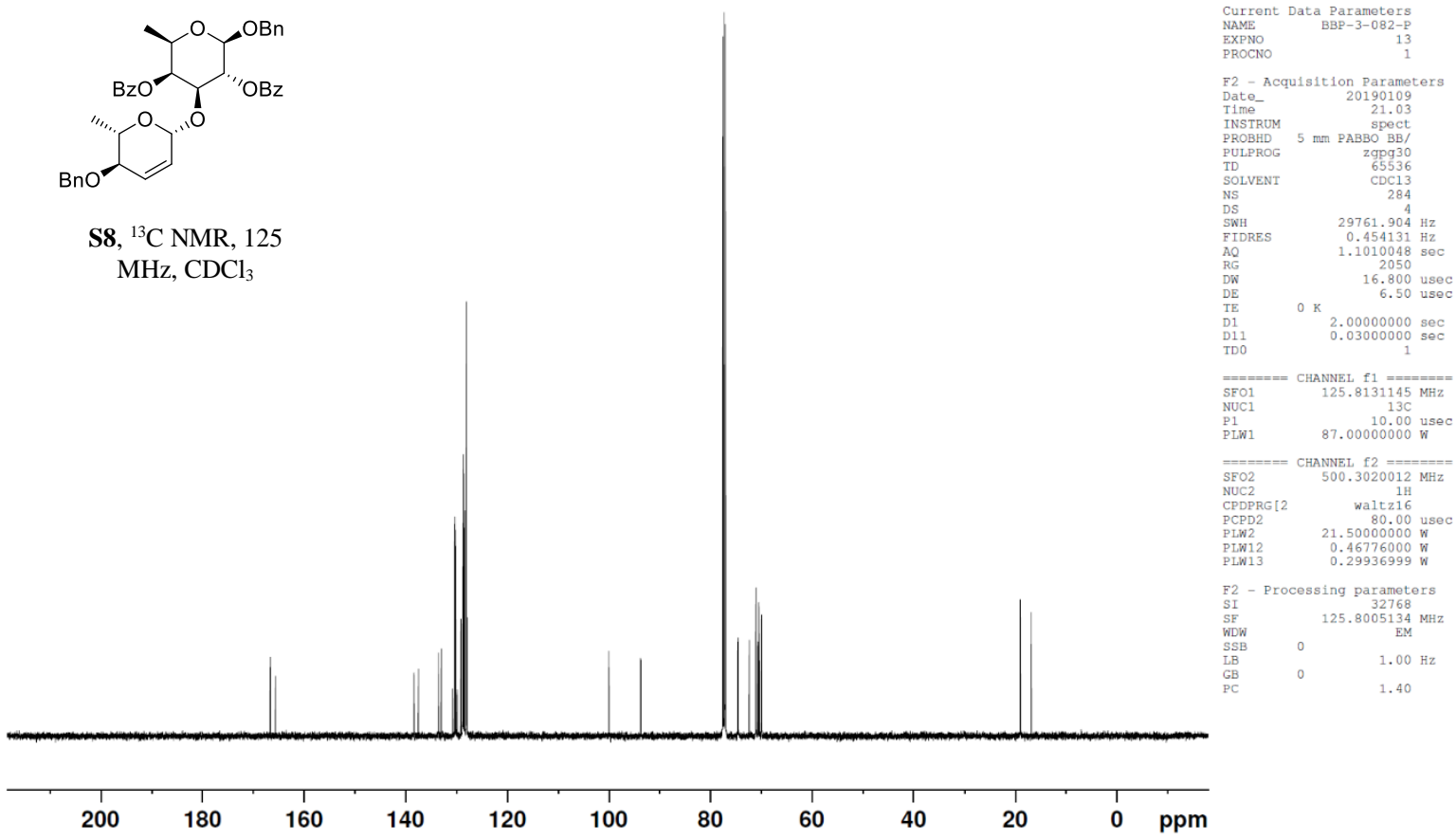